\title{
Genetic Targeting and Analysis of Parvalbumin and VGLUT3 Expressing Inhibitory Neurons
}

\author{
PhD Thesis \\ in partial fulfillment of the requirements \\ for the degree "Doctor rerum naturalium (Dr. rer. nat.)" \\ in the Molecular Biology Program \\ at the Georg August University Göttingen, \\ Faculty of Biology
}

submitted by

Christoph Bredack

born in

Lauchhammer, Germany

March 2011 



\section{Declaration}

Hereby, I declare, that the thesis "Genetic Targeting and Analysis of Parvalbumin and VGLUT3 Expressing Inhibitory Neurons" has been written independently and with no other sources and aids than quoted.

Christoph Bredack

Göttingen, March $31^{\text {st }}, 2011$ 


\section{Table of Content}

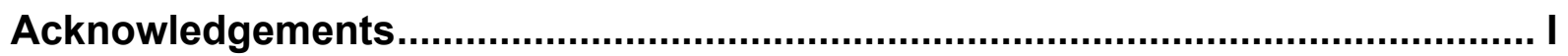

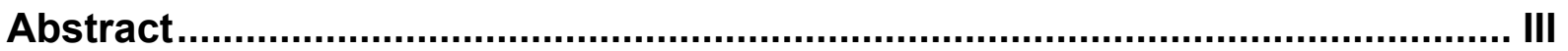

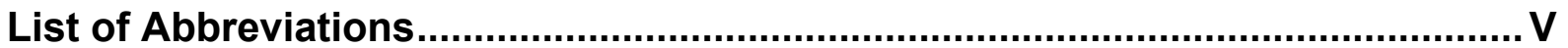

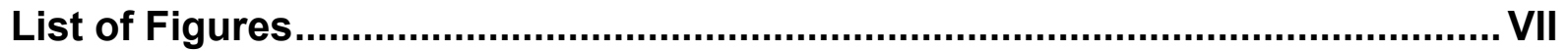

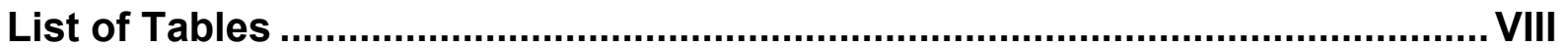

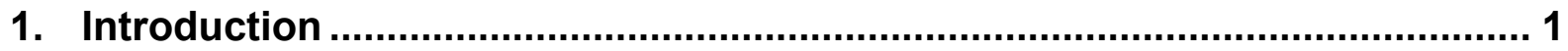

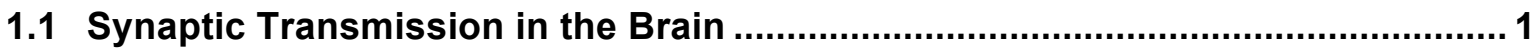

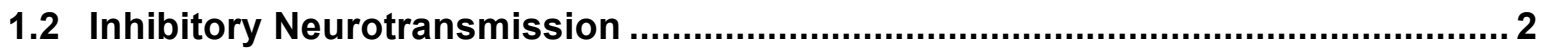

1.2.1 The Vesicular Inhibitory Amino Acid Transporter..........................................................

1.2.2 GABAergic Neurotransmission During Development ....................................................

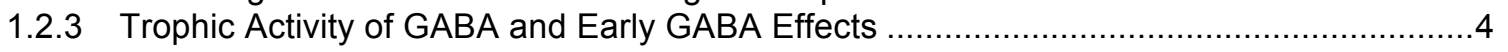

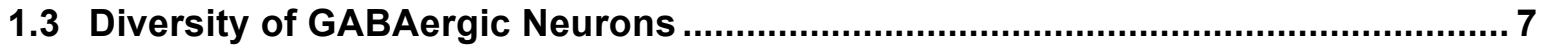

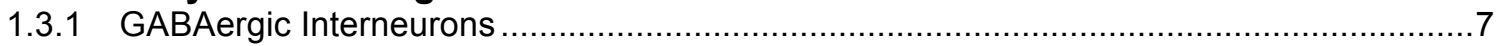

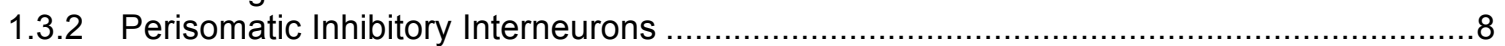

1.3.3 Projecting GABAergic Neurons - the Reticular Thalamic Nucleus ................................10

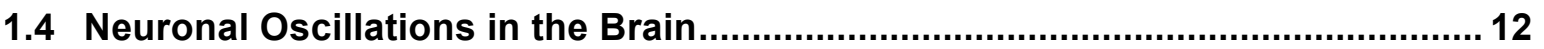

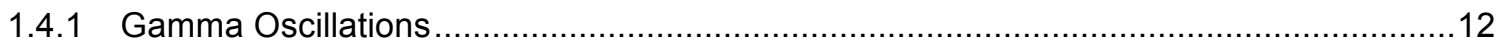

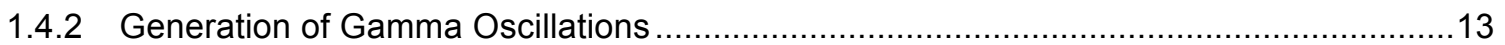

1.4.3 Firing Pattern of Distinct Cell Types During Gamma Oscillations .......................................15

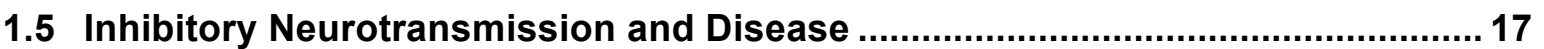

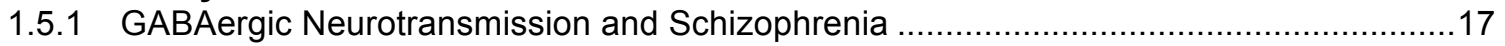

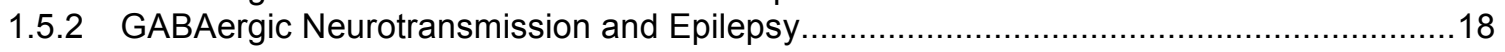

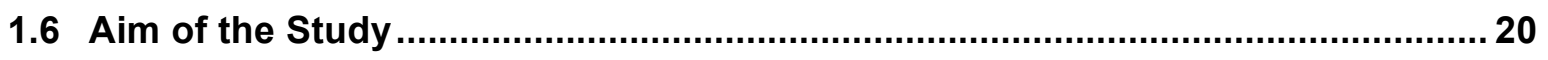

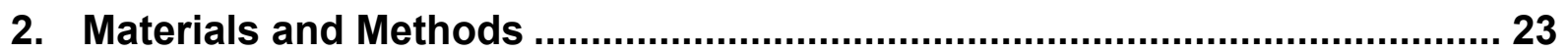

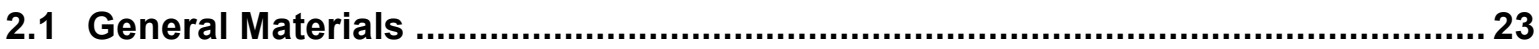

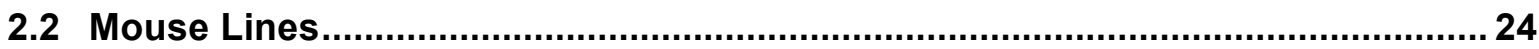

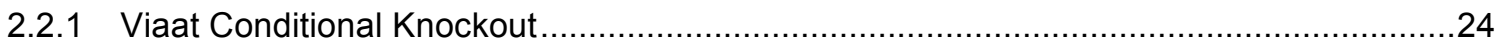

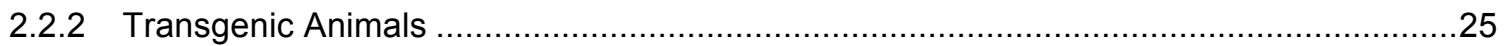

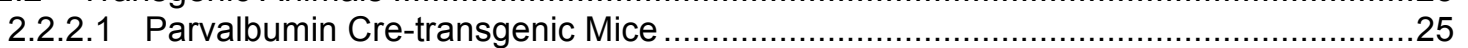

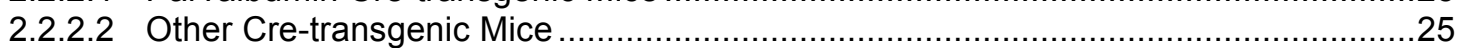

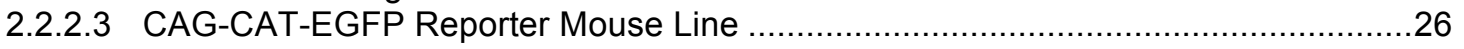

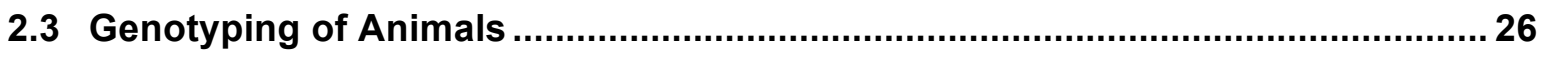

2.4 Tamoxifen Treatment of CreERT2-mouse Lines ............................................... 26

2.5 Immunofluorescence Analysis of Brain Sections ............................................ 27

2.6 Immunofluorescence Analysis of Cultured Primary Mouse Neurons ................. 28

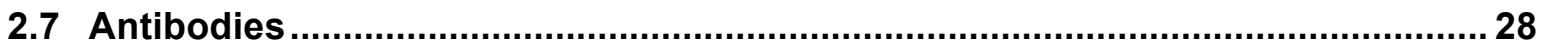

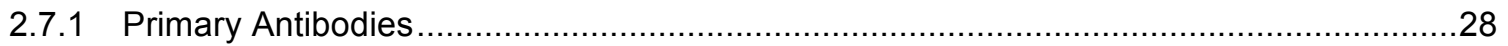

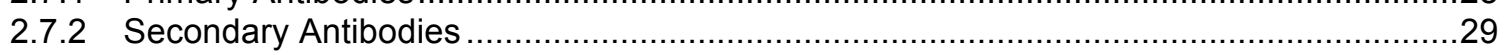

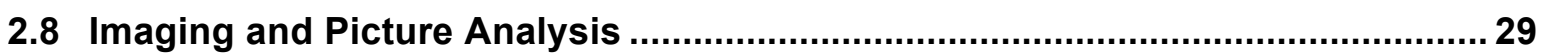

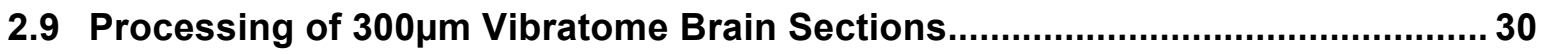

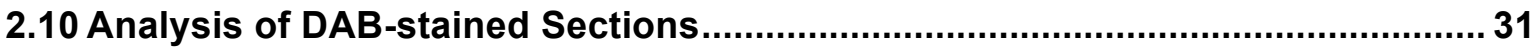

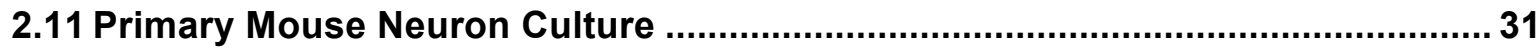

2.11.1 Continental Primary Mouse Neuron Cultures - Treatment of Coverslips …....................31 
2.11.2 Astrocyte Microisland Culture for Primary Mouse Neuronal Autaptic Cultures ................31

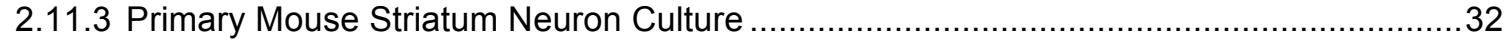

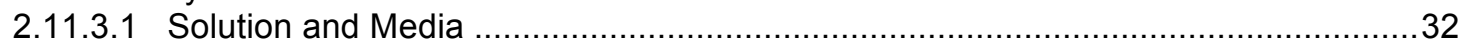

2.11.3.2 Preparation of Mouse Neuron Culture .................................................... 33

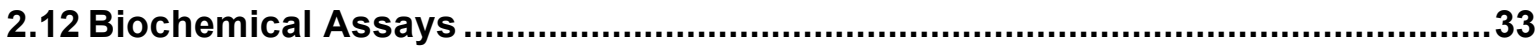

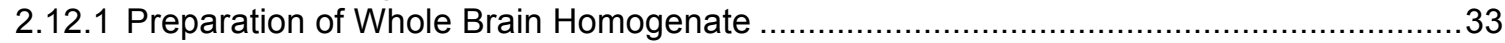

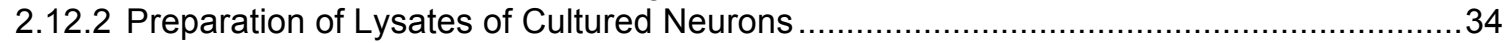

2.12.3 Sodium-Dodecyl-Sulfate-Polyacrylamid-Gel Electrophoresis (SDS-PAGE) ...................34

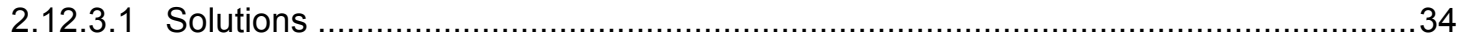

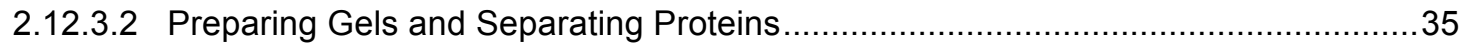

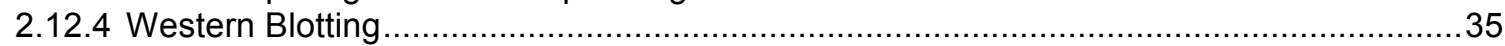

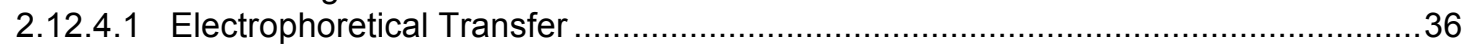

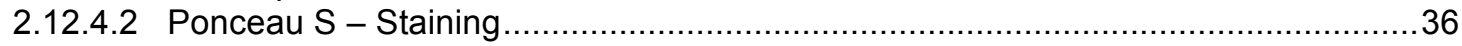

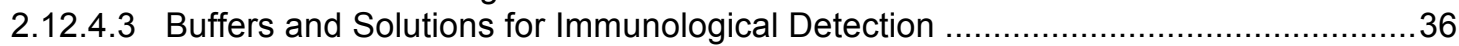

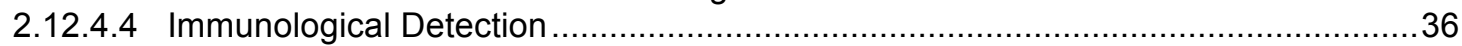

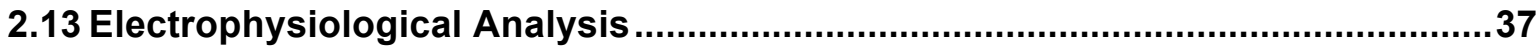

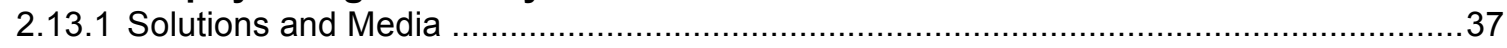

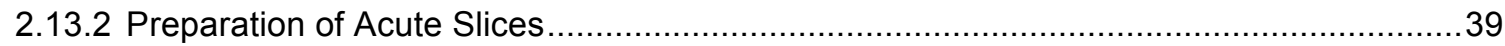

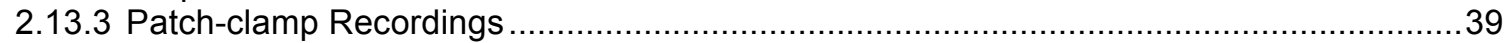

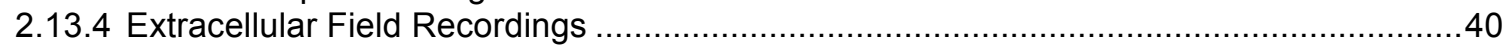

2.13.4.1 Gamma Oscillations in Submerged Conditions............................................40

2.13.4.2 Gamma Oscillations in Interface Conditions .............................................4 41

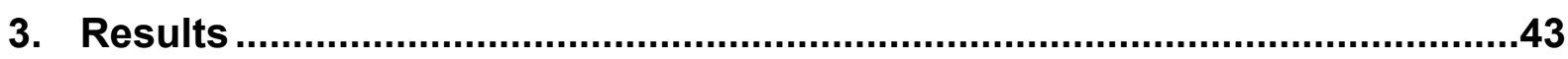

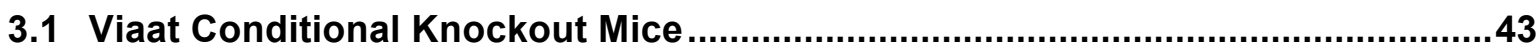

3.2 Shutdown of GABA Release in Cultured Viaat ${ }^{\mathrm{fl} / \mathrm{fl}}$ Neurons upon Expression of Cre-recombinase .............................................................................................4 44

3.3 Conditional Viaat Knockout in Parvalbumin Expressing Neurons .....................46

3.4 Expression Pattern of the PV-Cre Transgene .......................................................

3.5 Loss of Viaat in Viaat ${ }^{f l / f l}$, PV-Cre Neurons..................................................51

3.6 Morphological Consequences of Deleting Viaat in Parvalbumin Positive

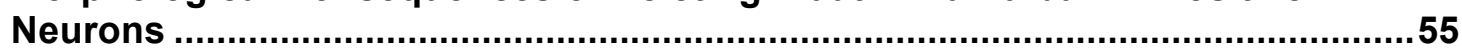

3.7 No Obvious Morphological Changes in Neurons of the Reticular Thalamic

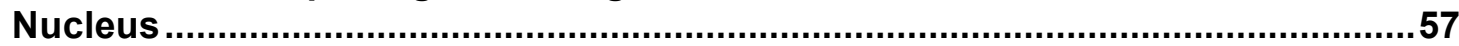

3.8 Unaltered Number of Parvalbumin Neurons ...................................................61

3.9 Spontaneous Miniature IPSCs were Unaltered in the nRT of Viaat ${ }^{\mathrm{f} / \mathrm{fl}}$, PV-Cre

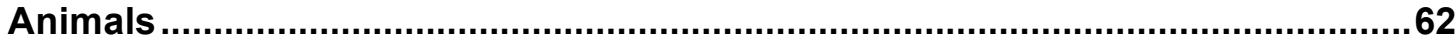

3.10 Altered Network Oscillations in Viaat ${ }^{\mathrm{fl} / \mathrm{fl}}, \mathrm{PV}$-Cre Animals...............................64

3.10.1 Gamma Oscillations in Submerged Conditions .................................................6.

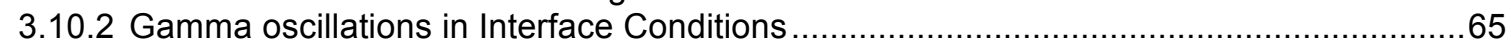

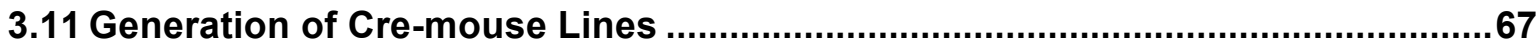

3.12 Analysis of the Conventional VGLUT3-Cre Mouse lines ....................................69

3.13 Analysis of Inducible Cre-mouse Lines.............................................................72

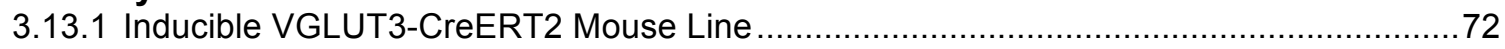

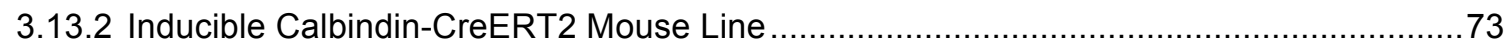

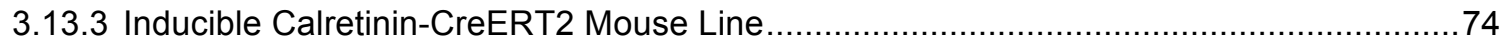

3.14 Contribution of VGLUT3 Expressing Interneurons to Gamma Oscillations .......75

4. Discussion ............................................................................................. 77

4.1 Analysis of Cre-recombinase Expression .......................................................78

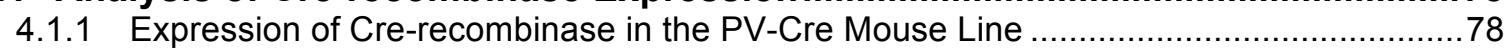


4.1.2 Expression Pattern of Cre-recombinase in Calbindin-, Calretinin- and VGLUT3-Cre Mouse Lines.

4.2 Motor - phenotype and Increased Seizure Susceptibility in Viaat ${ }^{\mathrm{fl} / \mathrm{fl}}$, PV-Cre Animals 82

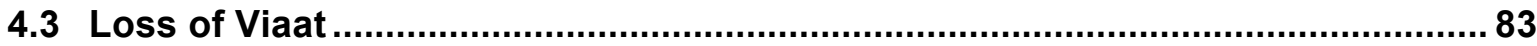

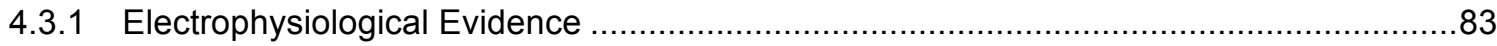

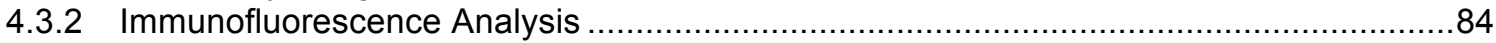

4.4 No Dramatic Morphological Consequences of Viaat Deletion in PV-expressing

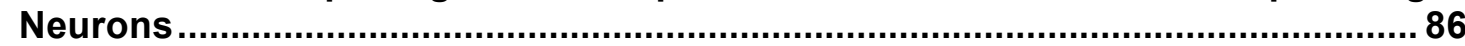

4.5 Decreased Gamma Oscillations in Viaat ${ }^{\text {fl/fl }}$, PV-Cre Animals ............................ 88

4.6 Reduced Gamma Oscillations in Viaat ${ }^{\text {fl/fl}}$, VGLUT3-Cre Animals .......................90

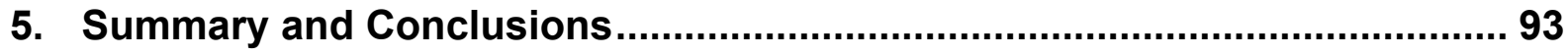

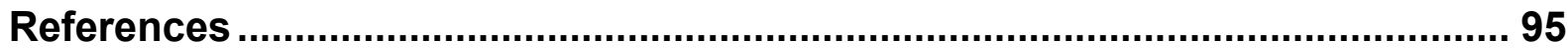

Curriculum Vitae ..................................................................................................... I 


\section{Acknowledgements}

This work would not have been possible and less fulfilling without the help of a lot of people and I am thankful for their support during the course of my PhD:

First of all, I want to thank my supervisor and first thesis committee member Dr. Sonja Wojcik. I am sincerely grateful for her scientific supervision, encouragement and all her help during every aspect of my PhD-work.

I want to thank Dr. JeongSeop Rhee for his help and supervision during all the electrophysiological studies within my PhD. I am indebted to Prof. Nils Brose, who welcomed me in his lab, for his guidance and critical input. I thank Prof. Erwin Neher and Prof. Ahmed Mansouri for their efforts and guidance as my thesis committee members.

I want to thank Anja Günther and Ines Beulshausen for their excellent technical support and in providing all the liters of solutions needed for the physiological experiments. Furthermore I thank Astrid Zeuch, Martin Dörre and Nina DankenbrinkWerder for their technical assistance in genotyping, sectioning and molecular biology work. I am grateful for the technical service provided by the AGCT-facility including Fritz Benseler, Dayana Schwerdtfeger and Ivonne Thanhäuser for their invaluable service of genotyping all the different mouse lines used in this study.

Thanks to all the friends and colleagues of the Molecular Neurobiology Department for their support, discussions and great moments shared in and outside of the lab. Without them, the PhD would not have been what it was.

I am grateful for external collaborators, especially the lab of Prof. Monyer for providing the PV-Cre mouse, one important part my PhD work. Furthermore Prof. Staiger and his lab for introducing me the neuron reconstructions and Neurolucida work, without them, this part of my study would have been much more difficult.

I am grateful to the IMPRS MSc/PhD program Molecular Biology and especially the coordination office team, Dr. Steffen Burkhardt, Ivana Bacakova and Kerstin Grüniger for their constant help and support during the years as a student in Göttingen.

I am deeply thankful for the friends outside of the lab for their support, the discussions about science and life as a PhD-student and the "non-scientific life". 
Finally and most importantly, I thank my family, who is always there and supported me throughout the whole time. 


\section{Abstract}

GABAergic interneurons are a highly diverse class of cell types that play essential roles in regulating the input/output behaviour of pyramidal neurons and of other interneurons. Thereby, these cells play a role in shaping neuronal network patterns and oscillations that are involved in higher brain functions like memory, attention and perception. Furthermore, GABAergic signalling is thought to play a crucial role in the development of single neurons and of neuronal networks.

A genetic approach was used to analyse the contribution of subsets of GABAergic interneurons to inhibitory neurotransmission. Mice carrying a loxP-flanked allele of the vesicular inhibitory amino acid transporter (Viaat) were crossed to mice expressing Cre recombinase under the control of the Parvalbumin (PV) and vesicular glutamate transporter 3 (VGLUT3) promoters. Since Viaat is essential for neurotransmitter release in GABAergic neurons, this approach allows the selective silencing of GABAergic inhibitory neurons expressing PV (Viaat $\left.{ }^{f / f t}, \mathrm{PV}-\mathrm{Cre}\right)$ and VGLUT3 (Viaat/flfl, VGLUT3-Cre), respectively. The contribution of these neurons to network function and morphological consequences of the cell type specific deletion of Viaat were analysed.

A loss of Viaat could be observed in several brain regions including the cerebellum and the reticular thalamic nucleus in Viaat/fl/fl, PV-Cre animals. However, the loss of Viaat from PV-expressing GABAergic neurons in these regions had no dramatic effect on the morphological development of the affected neurons.

PV- and VGLUT3-expressing GABAergic basket cells provide perisomatic inhibition to pyramidal neurons in the hippocampus, which plays a crucial role in the control of the output behaviour of these neurons. Therefore, the contribution of these two classes of GABAergic basket cells to the generation of hippocampal gamma oscillations was investigated in-vitro. The deletion of Viaat in PV-expressing neurons led to a reduction of the power of Kainate induced gamma oscillations in-vitro. Furthermore, the frequency of the maximum peak in the gamma field potential was significantly increased in mutant animals compared to control. Similar changes could be observed in Viaat ${ }^{f / / f l}$, VGLUT3-Cre animals. The power of gamma oscillations was reduced, although here the difference was statistically not significant, and the frequency of the maximum peak in the power spectrum was increased. 
These results confirm the importance of PV-expressing GABAergic interneurons in the generation of gamma oscillations in the hippocampus and provide evidence for the direct participation of VGLUT3-expressing hippocampal basket cells in the generation of these oscillations. 


\section{List of Abbreviations}

\begin{tabular}{|c|c|}
\hline$A B C$ & Avidin: Biotinylated enzyme Complex \\
\hline ACSF & Artificial cerebrospinal fluid \\
\hline AMPA & a-amino-3-hydroxy-5-methyl-4-isoxazolepropionic acid \\
\hline APS & Ammoniumpersulfate \\
\hline ATP & Adenosine-5'-triphosphate \\
\hline BAC & Bacterial artifical chromosome \\
\hline BCA & Bicinchoninic acid \\
\hline BSA & Bovine serum albumin \\
\hline CA1 & Cornu Ammonis area 1 \\
\hline CA3 & Cornu Ammonis area 3 \\
\hline CB & Calbindin \\
\hline CB1 & Cannabinoid receptor 1 \\
\hline $\mathrm{CCK}$ & Cholesystokinin \\
\hline CGCT & CAG-CAT-EGFP reporter allele \\
\hline $\mathrm{CR}$ & Calretintin \\
\hline CreERT2 & $\begin{array}{l}\text { Cre-estrogen receptor ligand binding domain mutant containing the } \\
\text { G400V/M543A/L540A mutation }\end{array}$ \\
\hline $\mathrm{DAB}$ & 3,3'-Diaminobenzidine \\
\hline DCN & Deep cerebellar nuclei \\
\hline DG & Dentate gyrus \\
\hline DHPG & (RS)-3,5-Dihydroxyphenylglycine \\
\hline DIV & Days in vitro \\
\hline DMEM & Dulbecco's Modified Eagle's Medium \\
\hline DNA & Deoxyribonucleic acid \\
\hline $\mathrm{E}$. & Embryonic day ... \\
\hline EDTA & Ethylenediaminetetraacetic acid \\
\hline EEG & Electroencephalography \\
\hline EGFP & Enhanced green fluorescent protein \\
\hline EGTA & 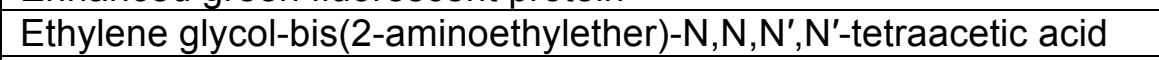 \\
\hline EPSC & Excitatory postsynaptic current \\
\hline ES-cells & Embryonic stem cells \\
\hline FCS & Fetal calf serum \\
\hline GA & Glutaraldehyd \\
\hline GABA & Gamma aminobutyric acid \\
\hline $\mathrm{GABA}_{\mathrm{A}} \mathrm{R}$ & GABA receptor class $A$ \\
\hline $\mathrm{GABA}_{\mathrm{B}} \mathrm{R}$ & GABA receptor class $B$ \\
\hline GAD65/67 & Glutamate decarboxylase of molecular weight $65 \mathrm{kDa} / 67 \mathrm{kDa}$ \\
\hline GDP & Giant depolarizing potential \\
\hline GTP & Guanosine-5'-triphosphate \\
\hline HBSS & Hank's Buffered Salt Solution \\
\hline HEPES & N-(2-Hydroxyethyl)piperazine-N'-(2-ethanesulfonic acid) \\
\hline $\mathrm{Hz}$ & Hertz \\
\hline IGEPAL & Octylphenoxypolyethoxyethanol \\
\hline$(\mathrm{m}) \mathrm{IPSC}$ & (miniature) Inhibitory postsynaptic current \\
\hline $\mathrm{Kb}$ & Kilobases \\
\hline KCC2 & $\mathrm{K}^{+}-2 \mathrm{Cl}^{-}$cotransporter \\
\hline $\mathrm{kg}$ & Kilogram \\
\hline $\mathrm{M}$ & Molar \\
\hline mAChR & Muscarinic acetylcholine receptor \\
\hline $\mathrm{mg}$ & Milligram \\
\hline
\end{tabular}




\begin{tabular}{|c|c|}
\hline mGluR & Metabotrophic glutamate receptor \\
\hline mIPSC & Miniature inhibitory postsynaptic current \\
\hline $\mathrm{ml}$ & Milliliter \\
\hline $\mathrm{mM}$ & Milli molar \\
\hline mOsm & Milliosmole \\
\hline mRNA & Messenger ribonucleic acid \\
\hline $\mathrm{mT}-\mathrm{mG}$ & Membrane targeted Tomato-membrane targeted EGFP double reporter allele \\
\hline $\mathrm{M} \Omega$ & Mega Ohm \\
\hline NA & Numerical aperture \\
\hline NBQX & 2,3-dihydroxy-6-nitro-7-sulfamoyl-benzo[f]quinoxaline-2,3-dione \\
\hline NKCC1 & $\mathrm{Na}^{+}-\mathrm{K}^{+}-2 \mathrm{Cl}^{-}$cotransporter \\
\hline NMDA & N-Methyl-D-Aspartat \\
\hline nRT & Reticular thalamic nucleus \\
\hline O-LM & Oriens-lacunosum moleculare \\
\hline $\mathrm{P} \ldots$ & Postnatal day ... \\
\hline PB-buffer & Phosphate buffer \\
\hline PBS & Phosphate buffered saline \\
\hline PCR & Polymerase chain reaction \\
\hline Pen/Strep & Penicillin/Streptomycin \\
\hline PFA & Paraformaldehyd \\
\hline PMSF & Phenylmethylsulfonylfluorid \\
\hline PoM & Posteromedial thalamic nucleus \\
\hline PV & Parvalbumin \\
\hline REM sleep & Rapid eye movement sleep \\
\hline RT & Room temperature \\
\hline SDS & Sodium dodecyl sulfate \\
\hline TB & Tris-buffer \\
\hline TBS & Tris buffered saline \\
\hline TC-neurons & Thalamocortical neurons \\
\hline TEMED & $\mathrm{N}, \mathrm{N}, \mathrm{N}$ ',N'-Tetramethyl-ethylenediamine \\
\hline Tris/HCl & Tris(hydroxymethyl)aminomethane hydrochloride \\
\hline TTX & Tetrodotoxin \\
\hline VB & Ventrobasal thalamus \\
\hline VGAT & Vesicular GABA transporter \\
\hline VGLUT1 & Vesicular glutamate transporter 1 \\
\hline VGLUT2 & Vesicular glutamate transporter 2 \\
\hline VGLUT3 & Vesicular glutatmate transporter 3 \\
\hline Viaat & Vesicular inhibitory amino acid transporter \\
\hline VIP & Vasoactive intestinal peptide \\
\hline VPL & Ventral posterolateral thalamic nucleus \\
\hline VPM & Posteromedial thalamic nucleus \\
\hline Z/EG & Beta Galactosidase/EGFP double reporter allele \\
\hline$\mu \mathrm{M}$ & Micro molar \\
\hline$\mu \mathrm{m}$ & Micrometer \\
\hline
\end{tabular}




\section{List of Figures}

Figure 3.1: Viaat Conditional Knockout Allele ........................................................

Figure 3.2: GABA Release is Eliminated in Cre-infected Viaat ${ }^{\mathrm{f} / \mathrm{fl}}$ Neurons ................45

Figure 3.3: Targeting of Parvalbumin Expressing GABAergic Neurons....................47

Figure 3.4: Expression of the PV-Cre Transgene in Different Brain Regions ............50

Figure 3.5: Loss of Viaat Immunoreactivity in Deep Cerebellar Nuclei and in the

Thalamus .53

Figure 3.6: No Obvious Loss of Viaat Immunoreactivity in the Hippocampus .54

Figure 3.7: No Obvious Morphological Consequences of the Loss of Viaat in the Deep Cerebellar Nuclei.

Figure 3.8: No Obvious Differences in the Axonic Morphologies of nRT Neurons

in Viaat ${ }^{\mathrm{flffl}}$, PV-Cre Mice Compared to Controls. .59

Figure 3.9: Neurolucida Reconstructions of nRT Neurons of Viaat ${ }^{\mathrm{fl} / \mathrm{fl}}$, PV-Cre Animals Compared to Control Mice. 61

Figure 3.10: Quantification of Parvalbumin Expressing Neurons.............................62

Figure 3.11: No change in mIPSC Amplitude or Frequency in the nRT ...................63

Figure 3.12: Unaltered Gamma Oscillations in Submerged Recording Conditions ...65

Figure 3.13: Reduced Hippocampal Gamma Oscillations in Viaat ${ }^{f / f / 1}$, PV-Cre Mice ...67

Figure 3.14: Constructs for the Generation of Cre-mouse Lines .68

Figure 3.15: Cre-expression in Line 1 of VGLUT3-Cre ${ }^{+}, \mathrm{CGCT}^{+}$Animals, which was Used for Further Experiments

Figure 3.16: Schematic Representation of Treatment of CreERT2-mice with Tamoxifen . .72

Figure 3.17: Expression of the Calbindin-CreERT2 Transgene _..............................73

Figure 3.18: Expression of the Calretinin-CreERT2 Transgene ...............................74

Figure 3.19: Reduced Gamma Oscillations in Viaat ${ }^{f / f 1}$, VGLUT3-Cre animals ............76 


\section{List of Tables}

Table 2.1: General Chemicals Used in this Study ................................................ 24

Table 2.2: General Material Used in this Study ................................................. 24

Table 2.3: Primary Antibodies Used in the Study................................................... 29

Table 2.4: Secondary Antibodies Used in this Study .......................................... 29

Table 2.5: Composition of Separating and Stacking Gel for SDS-PAGE ................. 35

Table 2.6: Overview of the Procedure for the Immunological Detection ................... 37

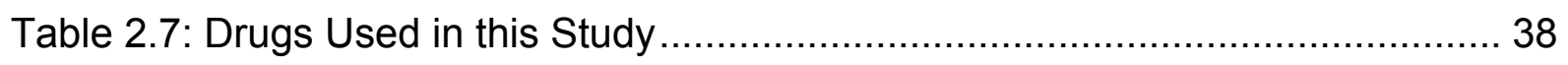

Table 3.1: Basic Parameters of the Somatodendritic Region of Reconstructed

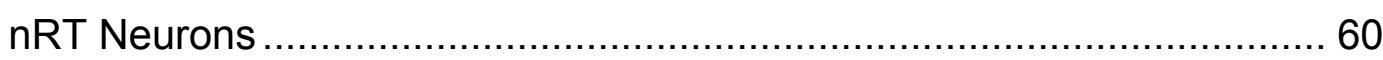




\section{Introduction}

\subsection{Synaptic Transmission in the Brain}

The human brain is the main computing and information processing unit in the human body. It is responsible for higher cognitive processes like memory, a wide array of human emotions, performance of precise and highly controlled movements and logical reasoning that allows humans to solve complex questions. These complex performances rely on the correct development and wiring of the neurons of the central nervous system. Damages to the brain, which leads to loss of neurons as well as small changes in the developmental programme of these cells, can disturb the function of the brain profoundly and can lead to the collapse of the highly regulated and defined system. The functionality of the brain depends on the contact points between its composing neurons, the synapses, from the Greek "syn-" ("together") and "haptein" ("to clasp") as defined by Sir Charles Scott Sherrington and Michael Foster in 1897. One hundred billion nerve cells are interconnected by 100 trillion synapses. A synapse is an asymmetrical cell-contact site consisting of a presynaptic side, containing the release machinery for the neurotransmitter and the postsynaptic side, with the recognition complex for the neurotransmitter. The preand postsynaptic side are separated by an around $20 \mathrm{~nm}$ wide synaptic cleft.

The release of neurotransmitter, which is stored in synaptic vesicles, is the main form of communication between nerve cells. At a chemical synapse the incoming electrical signal in form of an action potential is converted into a chemical signal and transmitted to the next neuron. On the presynaptic side, the action potential induces the opening of voltage-dependent calcium channels and thereby induces the calcium dependent exocytosis of fusion competent synaptic vesicles at the membrane of the presynaptic active zone (Sudhof, 2004). This allows the release of neurotransmitter into the synaptic cleft. The neurotransmitter diffuses through the cleft, binds to receptors on the postsynaptic side and activates them. This activation induces a post-synaptic response, that can be excitatory, by allowing the influx of cations, leading to a depolarisation of the membrane or inhibitory by opening $\mathrm{Cl}^{-}$-channels, which leads to a hyperpolarisation of the postsynaptic membrane (reviewed by Fritschy and Brunig, 2003; Mody and Pearce, 2004; Okabe, 2007). The fusion of

synaptic vesicles only occurs at the active zone, a specialised membrane region in the presynaptic terminal precisely aligned with the postsynaptic density, a specialised 
region in the postsynapse to allow the precise communication between nerve cells (Fernandez-Chacon and Sudhof, 1999; Garner et al., 2002).

\subsection{Inhibitory Neurotransmission}

Gamma-amino butyric acid (GABA) and glycine are the main inhibitory neurotransmitters in the central nervous system. GABAergic neurotransmission acts predominantly in the brain (Krnjevic and Schwartz, 1967) whereas both glycinergic (Werman et al., 1968) and GABAergic inhibition occur in the brain stem and spinal cord (Jonas et al., 1998; O'Brien and Berger, 1999).

\subsubsection{The Vesicular Inhibitory Amino Acid Transporter}

Vesicular release of neurotransmitter requires its prior accumulation in synaptic vesicles. Only one vesicular transporter for GABA and glycine is known - the vesicular inhibitory amino acid transporter (Viaat) (Sagne et al., 1997) or vesicular GABA transporter (VGAT) (McIntire et al., 1997). The gene for Viaat spans around $5 \mathrm{~kb}$ in mouse and human and is organized in two exons. These two exons encode for a large, hydrophilic cytosolic N-terminus (140 amino acids) and a 400 amino acid core, forming ten putative transmembrane domains connected by shorter loops (Gasnier, 2004; McIntire et al., 1997; Sagne et al., 1997). Viaat is the only member of the SLC32 family of transporters and belongs to the eukaryotic superfamily of $\mathrm{H}^{+}$coupled amino acid transporters together with SLC36 (proton coupled amino acid transporter) or SLC38 (sodium coupled neutral amino acid transporter) (Gasnier, 2004). The lumen of synaptic vesicles gets positively charged and acidified by the activity of the vacuolar $\mathrm{H}^{+}$-ATPase. Viaat acts as an $\mathrm{H}^{+}$/neurotransmitter antiporter and exchanges vesicular protons against cytosolic GABA and glycine, which allows their subsequent exocytotic release.

Viaat is the only known vesicular transporter for GABA and glycine. The transporter is co-expressed with markers for GABAergic and glycinergic synapses including GABA and glycine itself, the GABA-synthesizing enzymes glutamate decarboxylase 65 and 67 (GAD65 and 67) and the membrane glycine transporter GlyT2 (Chaudhry et al., 1998; Dumoulin et al., 1999; Sagne et al., 1997). Biochemical studies showed, that Viaat is able to transport both GABA and glycine into synaptic 
vesicles. Heterologously expressed Viaat protein in PC12 or COS7 cells shows a prominent GABA transport activity. The affinity for glycine was significantly lower (Mclntire et al., 1997; Sagne et al., 1997). However uptake studies with endogenous vesicle preparations show a similar uptake rate for GABA and glycine (Christensen and Fonnum, 1991). Electrophysiological studies show the co-release of GABA and glycine from spinal cord neurons (Jonas et al., 1998; O'Brien and Berger, 1999; Wojcik et al., 2006), indicating the same transport mechanism.

The final proof, that Viaat is the only transporter for GABA and glycine, is given by the genetic deletion of Viaat in mice. Viaat knockout neurons from striatum (GABAergic) and spinal cord (mixed GABAergic/glycinergic) are synaptically silenced and show no release of GABA or glycine (Wojcik et al., 2006), which demonstrates that GABAergic and glycinergic neurons depend on the same vesicular transporter. A partial loss of GABA synthesizing enzyme from spinal cord synapses is apparent in the Viaat knockout, which indicates a close association of the transporter and the enzyme providing the transported molecule. Therefore the co-expression of Viaat and the GAD enzymes determines the GABAergic phenotype for a cell (Wojcik et al., 2006), whereas, the co-expression of Viaat with the neuronal membrane glycine transporter GlyT2 is responsible for the glycinergic phenotype of a neuron (Gomeza et al., 2003).

The relevance of co-release of GABA and glycine in mixed synapses of the spinal cord, especially in developing spinal cord (Gao et al., 2001) and the brain stem, remains to be elucidated. In the auditory brainstem pathway, where a fast inhibitory regulation is necessary, it might enhance the postsynaptic glycinergic activity (Lu et al., 2008). However, a functional requirement for GABA signalling outside of the synaptic cleft is also possible. For example, it might provide a regulatory feedback mechanism through the activation of presynaptic $\mathrm{GABA}_{\mathrm{B}}$ receptors, thereby causing a reduction in presynaptic glycine release (Lim et al., 2000).

\subsubsection{GABAergic Neurotransmission During Development}

GABA and glycine both act as inhibitory neurotransmitter in the mature nervous system. The intracellular $\mathrm{Cl}^{-}$concentration in mature neurons tends to be low (around $7 \mathrm{mM}$ (Ben-Ari, 2002)). The opening of $\mathrm{GABA}_{A}$-receptors $\left(G A B A_{A} R\right)$ or glycine 
receptors (GlyR) upon binding of the respective neurotransmitter therefore leads to $\mathrm{Cl}^{-}$influx, membrane hyperpolaristion and thus inhibition of the postsynaptic neuron.

In immature neurons the intracellular $\mathrm{Cl}^{-}$concentration is high enough (around $20-30 \mathrm{mM}$ in young neurons (Owens et al., 1996) to lead to $\mathrm{Cl}^{-}$efflux upon $\mathrm{GABA}_{A} \mathrm{R}$ or GlyR opening. GABA and Glycine thus depolarize immature neurons. The membrane depolarization can be sufficient for the opening of voltage dependent $\mathrm{Ca}^{2+}$ channels, which can trigger an action potential (Ben-Ari et al., 1989; Ben-Ari et al., 2007; Leinekugel et al., 1997; Leinekugel et al., 1995; Owens et al., 1996; Staley and Smith, 2001). The high intracellular chloride concentration in immature neurons is due to the early expression of the inward directed $\mathrm{Na}^{+}-\mathrm{K}^{+}-2 \mathrm{Cl}^{-}$cotransporter NKCC1 (Clayton et al., 1998). During development, the expression of the $\mathrm{K}^{+}-2 \mathrm{Cl}^{-}$ cotransporter $\mathrm{KCC} 2$, which is responsible for lowering the intracellular chloride concentration, is upregulated (Rivera et al., 1999). This causes the switch from excitatory GABA/glycine neurotransmission to inhibition. The developmental timing of this excitatory-inhibitory switch is highly dependent on the species and brain structure. The upregulation of KCC2 and the switch to inhibitory GABA activity is highly correlated with the maturation of neurons (Stein et al., 2004; Yamada et al., 2004). It has been proposed, that the developmental upregulation of the outward directed $\mathrm{Cl}^{-}$-transporter is activity dependent, mediated by the early excitatory, GABAergic activity (Ganguly et al., 2001). However, further in-vitro studies (Ludwig et al., 2003; Titz et al., 2003) and the silencing of GABAergic neurons in-vivo by deleting the gene for Viaat (Wojcik et al., 2006) showed, that GABA mediated activity is not necessary for the correct developmental expression pattern of KCC2. The upregulation of expression of $\mathrm{KCC} 2$, hence the switch to inhibitory neurotransmission, occurs mainly postnatally in rodents (Gulyas et al., 2001; Ludwig et al., 2003). However, KCC2 function is essential early in postnatal development, since the KCC2 deletion mutant dies right after birth (Hubner et al., 2001).

\subsubsection{Trophic Activity of GABA and Early GABA Effects}

GABA is a neurotransmitter that mainly exerts its inhibitory or excitatory function after vesicular release from synapses. However, early in development, before functional synapses are formed, GABA acts as a trophic substance with a variety of effects on the development and maturation of the brain and on the formation of 
neuronal circuits (Represa and Ben-Ari, 2005). This early function of GABA might be generated by a non-vesicular, diffuse release of GABA in the developing neuronal structures (Demarque et al., 2002).

The activation of $\mathrm{GABA}_{\mathrm{A}}$-receptors leads to a decrease of DNA synthesis in cortical, proliferating cells (LoTurco et al., 1995). This activity of GABA occurs highly interconnected with the activity of different growth factors like basic fibroblast growth factor (bFGF) (Antonopoulos et al., 1997). An early influence of GABA on the proliferation of progenitor cells can be observed early in development and may therefore affect cortical cell composition (reviewed by Owens and Kriegstein, 2002b). Furthermore, GABA affects the migration of neuroblasts and can modulate their behaviour. In-vitro studies show, that the activation of $\mathrm{GABA}_{B}$ receptors alters the migration of future GABAergic interneurons (Lopez-Bendito et al., 2003). GABA can either act as a motility-promoting factor on the migration of neuroblasts or as a stop signal (Behar et al., 1998; Heck et al., 2007). Therefore it can influence the correct layering of brain structures. However, in knockout studies where genes essential for the GABAergic neurotransmitter system were deleted, i.e. GAD65/67 (Ji et al., 1999), Viaat (Wojcik et al., 2006) or KCC2 (Hubner et al., 2001), early histogenesis of the brain was not impaired. This might be explained by compensatory activities of other neurotransmitter system, like the glutamatergic system or by the early lethality of these mouse models. However, a detailed analysis of potential morphological deficits of neurons was not performed in these studies. Further studies show diverse effects of GABA signalling on the morphological development of neurons in the developing and mature central nervous system. A premature induced switch of the excitatoryinhibitory GABA signalling, leads to maturation defects of neurons with fewer, shorter and less branched dendrites (Cancedda et al., 2007) and shows a crucial effect of GABA signalling on the development of neurons. Furthermore, early perturbations of GABA synthesis leads to less pronounced axonal arborisations and a reduced synapse formation of cortical PV-expressing cells (Chattopadhyaya et al., 2007). The effects of GABA on the morphological development of neurons are mediated by $\mathrm{Ca}^{2+}$-signalling (Ageta-Ishihara et al., 2009). A GABA mediated increase of intracellular $\mathrm{Ca}^{2+}$ influences kinase cascades that are involved in the regulation of axon growth.

GABAergic input is crucial in early neuronal circuits, since the development of GABAergic synapses precedes the development of glutamatergic contact sites (Tyzio 
et al., 1999). Application of $\mathrm{GABA}_{\mathrm{A}}$ receptor antagonists blocks all the activity in immature hippocampal slices (Ben-Ari et al., 1989). This indicates that GABA is the predominant neurotransmitter in the immature hippocampus and that GABAergic synapses are active before glutamatergic ones. Furthermore, in immature P0 hippocampal slices, three types of neurons can be analysed electrophysiologically (i) silent neurons, (ii) neurons with GABAergic postsynaptic currents and (iii) neurons with mixed GABAergic and glutamatergic postsynaptic responses. These three types of neurons correspond to morphological maturation states, indicating that GABAergic synapses are formed first on less developed, younger neurons and, glutamatergic contacts are formed only later on more developed cells (Ben-Ari, 2002; Ben-Ari et al., 1989; Ben-Ari et al., 2007; Tyzio et al., 1999). The first circuit activity in the hippocampus, the giant depolarizing potentials (GDPs), is generated by GABAergic signalling. GDPs are long lasting $(300 \mathrm{~ms})$ depolarizing potentials with a frequency of around $0.1 \mathrm{~Hz}$ that can be blocked by the application of GABA receptor antagonists but are also influenced by NMDA receptor antagonists (Ben-Ari et al., 1989). This indicates that depolarizing GABA signalling is important for the generation of this early network activity in the hippocampus, which is modulated by NMDA receptor responses. GDPs occur in acute hippocampal slices, all subfields of the hippocampus and neuron types. Furthermore they are observed in-vivo and in intact hippocampus preparations. This activity can be recorded in different brain structures like the cortex, cerebellum, spinal cord and a similar activity is observed in the retina in rodents and other species (reviewed by Ben-Ari, 2002; Ben-Ari et al., 2007). They are highly correlated with the excitatory activity of GABA and disappear with the excitatory-inhibitory switch, in rodents around the second postnatal week (Ben-Ari et al., 1989), in primates already before birth (Khazipov et al., 2001). After this, more complex circuit activity, like theta and gamma oscillations start to occur. The early GABA driven activity is important for the generation of early neuronal networks and for the integration of neurons in developing circuitries in the brain (Ben-Ari, 2008a; Ben-Ari et al., 2007; Leinekugel et al., 1997). The integration of newly born neurons in existing neuronal networks also depends on the excitatory GABA signalling and a perturbation of the signalling leads to a disturbed development of the newborn neuron and a misintegration into the network (Ge et al., 2006). 


\subsection{Diversity of GABAergic Neurons}

Different types of neurons exist in the brain. Principal neurons or projection neurons possess long axons and send their information to other brain regions or outside of the brain. Nonprincipal neurons or interneurons, which constitute around $20 \%$ of neocortical neurons, form local axonic arborisations and usually do not project outside of their brain region. One prominent class of interneurons are GABAergic interneurons that provide the main source of inhibition in the brain and can form highly connected networks (Markram et al., 2004; McBain and Fisahn, 2001).

\subsubsection{GABAergic Interneurons}

GABAergic interneurons are by no means a homogenous class of cells. However, some common features distinguish them from pyramidal neurons: including the usually non-spiny dendrites, the presence of GABAergic and glutamatergic synapses on the soma and processes of the cells and the local axonal arborisation of most of the interneurons. However different classes of GABAergic interneuron innervate distinct domains of target neurons (summarized by Markram et al., 2004).

A wide variety of GABAergic interneurons can be classified based on their morphological features, electrophysiological properties and the expression profiles of certain marker proteins. The simple description of the axonic and dendritic processes (e.g. basket cells), the postsynaptic target region (axo-axonic, bistratisfied) or the location of the soma and the axonal target region (e.g. stratum oriens-lacunosum moleculare - O-LM cells) can be used to classify groups of neurons. Taking the physiological and synaptic properties into account, leads to further classification schemes based on the firing pattern. Originally all GABAergic interneurons were classified as fast-spiking neurons (Connors and Gutnick, 1990; McCormick et al., 1985), but further discharge patterns have been described, such as low-thresholdspiking or late-spiking cells (Kawaguchi and Kubota, 1997). Furthermore, the expression of marker proteins can be used to describe subsets of GABAergic interneurons. The expression of calcium-binding proteins like Calbindin (CB), Calretinin ( $C R$ ) and Parvalbumin (PV), neuropeptides like vasoactive intestinal peptide (VIP) or Cholesystokinin (CCK) or other vesicular proteins, like the vesicular glutamate transporter 3 (VGLUT3) can be used to classify interneurons. However, 
GABAergic interneurons show a great diversity in their morphological and electrophysiological properties and the expression of marker proteins is often overlapping (for review see Freund and Buzsaki, 1996; Fukuda and Kosaka, 2000; Klausberger and Somogyi, 2008; Maccaferri and Lacaille, 2003; Markram et al., 2004).

For example, PV is expressed in distinct morphological classes of cells. In the hippocampus, basket, axo-axonic, bistratisfied and O-LM cells can be distinguished, that represent different innervation patterns of the postsynaptic targets. The basket cells (Marin-Padilla, 1969) and axo-axonic cells (Somogyi, 1977) innervate predominantly the perisomatic region of pyramidal cells, whereas bistratisfied cells innervate the pyramidal cell dendrites in the stratum oriens and radiatum and O-LM cells the dendrites in the stratum lacunosum-moleculare (see Klausberger, 2009). Another class of cells, expressing CCK, provides perisomatic inhibition in parallel to the PV-expressing basket cells (Freund and Katona, 2007). These CCK positive neurons also express either VIP (Acsady et al., 1996) or VGLUT3 in the hippocampus and cortex (Somogyi et al., 2004). This example shows, the same morphological type of neuron can show different expression patterns of marker proteins or expression of the same marker protein can be observed in different morphological cell types. Thus, for the description and classification of GABAergic interneurons morphological, cell biological and physiological characteristics need to be taken into account (Ascoli et al., 2008).

\subsubsection{Perisomatic Inhibitory Interneurons}

Interneurons innervating the perisomatic regions of a pyramidal neuron are positioned to control the output behaviour of target neurons and therefore to synchronize the firing pattern of a larger group of neurons (Cobb et al., 1995). Two subclasses of perisomatic targeting neurons can be described in cortex and hippocampus - the PV- and CCK-expressing basket cells (Freund and Buzsaki, 1996). Even though both cell types innervate and provide inhibition to the same domain of a pyramidal cell, both types differ substantially in their expression pattern of receptors, transmitters, modulators, as well as input received and the firing pattern. Therefore, the different types of basket cells are considered to fulfill different functions in perisomatic inhibition. PV-expressing basket cells provide the overall 
rhythm of network activity, whereas the CCK cells act as a fine-tuning unit (reviewed by Freund, 2003; Freund and Katona, 2007). PV-expressing interneurons receive more glutamatergic synapses (Gulyas et al., 1999), have larger excitatory postsynaptic currents (EPSC) amplitude responses and have short membrane time constants (Glickfeld and Scanziani, 2006). They show a more precise neurotransmitter release than CCK-expressing basket cells (Hefft and Jonas, 2005) and a fast-spiking firing pattern (Pawelzik et al., 2002). All these features allow them to respond to input quickly, which is crucial for the generation of rhythmic activity in neuronal circuits (Freund and Katona, 2007). Compared to PV-positive basket cells, CCK cells receive less glutamatergic but more GABAergic input, for example from interneuron specific CR-expressing neurons (Gulyas et al., 1996; Matyas et al., 2004). CCK-expressing basket cells have a longer membrane time constants, which allows them to integrate input signals over a wider time window (Glickfeld and Scanziani, 2006). Their firing pattern is regular-spiking (Pawelzik et al., 2002) and a rather asynchronous transmitter release is a characteristic feature (Hefft and Jonas, 2005). CCK-expressing basket cells receive prominent serotonergic input from the medial raphe (Morales and Bloom, 1997; Varga et al., 2009). All of these features put them in a regulatory position to integrate inputs from several sources, generating long-lasting inhibitory input, which is important for the tuning of rhythmic activity (Freund and Katona, 2007). Furthermore, different receptors are expressed on the presynaptic terminals of the two cell types. CCK-positive cells express the cannabinoid receptor CB1 (Marsicano and Lutz, 1999), whereas PV-expressing basket cells express $\mu$-opioid receptors in their terminals (Drake and Milner, 2002; Neu et al., 2007). CB1-receptor activation can reduce the neurotransmitter release of the presynaptic cell by depolarization-induced suppression of inhibition (DSI), by release of endocannabinoids from the target cells that can silence the CCKexpressing cell (Wilson and Nicoll, 2001). The same is true for PV-expressing cells. The activation of opioid receptors leads to a reduction of GABA release from these neurons (Drake and Milner, 2002; Svoboda et al., 1999). All the above mentioned differences between these two cell types are in a agreement with the theory of their distinct functions: the PV-expressing cells provide the overall rhythm, whereas CCKexpressing basket cells mediate subcortical and local information, which is important for in-vivo rhythmic neuronal activity (Freund, 2003; Freund and Katona, 2007). 


\subsubsection{Projecting GABAergic Neurons - the Reticular Thalamic Nucleus}

Not all GABAergic neurons are local circuit neurons (see above), projecting inhibitory neurons can be found throughout the brain. For example, GABAergic neurons with long projecting axons occur in the hippocampus. These cells innervate neurons of the hippocampus (pyramidal and GABAergic cells) but also project outside of the hippocampus. Trilaminar cells for instance send their processes within the hippocampal formation but also to the subiculum (Sik et al., 1995), double projection cells send long axons to the subiculum and to the medial septum (Jinno et al., 2007). Other classes of projecting GABAergic neurons include the cerebellar Purkinje cells, which represent the main inhibitory output of the cerebellum and innervate cells of the deep cerebellar nuclei (Teune et al., 1998).

Neurons of the reticular thalamic nucleus (nRT) project outside of its region into the underlying thalamus. The reticular thalamic nucleus forms a shell-like structure surrounding the dorsal thalamus, which is positioned at the interface between the thalamus and the internal capsule. Therefore, it is in a perfect position to control the thalamo-cortical pathways and is involved in the regulation of gating and processing of information from the thalamus to the cortex (for review see Fuentealba and Steriade, 2005; Pinault, 2004). The nRT is composed of PV-expressing (Frassoni et al., 1991), GABAergic (Houser et al., 1980) projecting neurons. Different studies of nRT neurons disagree on whether the nRT consists of a homogenous or heterogeneous group of cells based on their somato-dentritic appearance (Lubke, 1993; Ohara and Havton, 1996; Spreafico et al., 1991). However three different types have been described: (i) cells with dendritic processes in the dorso-ventral axis, (ii) cells with ramifications in the rostro-caudal orientation and (iii) cells with dendritic processes in the all directions (Pinault and Deschenes, 1998). The cells also show a wide variety of axonal projection towards the thalamus, but never project to the cortex. The axon originates from the soma or from a dendrite and penetrates the thalamus perpendicular to the interface of the $\mathrm{nRT}$ and thalamus. Axons show a defined arbor with a clear synaptic field (Pinault and Deschenes, 1998). In the VPM region three different kind of synaptic fields have been described in young rats: clustered, intermediate and diffuse synaptic tuft (Cox et al., 1996). Cells send their axons into all nuclei of the thalamus in a more or less parallel manner i.e. a single cell projects towards one specific thalamic nucleus. However, an nRT cell is also able to innervate two different thalamic nuclei (Pinault and Deschenes, 1998). Neurons of 
the nRT form a highly interconnected network within the nucleus by forming dendrodentric synapses, axo-dendritic synapses and gap-junctions (Cox et al., 1996; Pinault, 2004; Pinault et al., 1997; Zhang and Jones, 2004).

Cells of the $\mathrm{nRT}$ receive direct excitatory input from thalamocortical (TC) relay neurons (Harris, 1987). This thalamocortical input and the corresponding response of nRT cells leads to a direct inhibition of TC-neurons but might also disinhibit these cells because of inhibited local inhibitory circuit neurons in the thalamus. Furthermore, a massive input into the $\mathrm{nRT}$ is formed by cortico-thalamic collaterals passing through the nRT (Liu and Jones, 1999). Activation of nRT neurons through cortical input in turn increases inhibitory events in TC cells and local interneurons (for review see (Steriade, 1999)). Furthermore, the nRT receives GABAergic input from the globus pallidus or the substantia nigra pars reticularis (Cooper and Stanford, 2002; Cornwall et al., 1990; Gandia et al., 1993; Lee and Tepper, 2007; Pare et al., 1990), cholinergic and monoaminergic input from the brain stem (Kolmac and Mitrofanis, 1998) and basal forebrain (Hallanger et al., 1987). Thus, the nRT is under constant control from cortical, thalamic and subcortical input.

The nRT plays an important role in the generation of spindle oscillations (7$14 \mathrm{~Hz})$. This rhythmic activity occurs during early sleep phases and also in the isolated nRT (Steriade et al., 1987). Sleep spindles are thought to be important for synaptic plasticity in the thalamus and cortex and may be involved in memory consolidation (Steriade and Timofeev, 2003). Spindles in the nRT are generated by low threshold currents resulting in bursting activity of $\mathrm{nRT}$ neurons during sleep phases (Steriade, 2005; Steriade et al., 1987; Steriade et al., 1993). This activity is spread in the nucleus via intrareticular synapses and electrical coupling. The activation of a subset of nRT cells by cortico-thalamic input, which spreads throughout the nRT and leads to synchronized rhythmic firing, can induce spindles, as well (Steriade, 2005; Steriade et al., 1993).

The $\mathrm{nRT}$ is also involved in pathophysiological processes like the generation of absence seizures. Spike-wave (SW) discharges with a frequency of around $3-4 \mathrm{~Hz}$ are generated in the cortex during such events (Steriade and Contreras, 1998). The nRT-cells follow every discharge in the cortex with the generation of inhibitory potentials in the TC neurons (Steriade and Contreras, 1995). Therefore, the TC cells are inhibited throughout this activity, which prevents the transfer of information to the 
cortex. The cortex is therefore disconnected from the "outside" world, which serves as an explanation for the consciousness impairment during absence seizures (see Steriade, 2005).

\subsection{Neuronal Oscillations in the Brain}

The generation of rhythmic neuronal activity is characteristic for networks of neurons. Mutual excitation can generate a rhythmic, organized firing of a lager group of cells, which can generate large discharges for example in epilepsy. Regulation and limitation of this rhythmic activity is performed by GABAergic inhibitory interneurons. Neuronal oscillations occur in several brain regions including the cortex, hippocampus, olfactory bulb and thalamus. Oscillations can occur in several frequency ranges from slow oscillation in the delta $(0.5-3 \mathrm{~Hz})$ and theta range $(3-$ $8 \mathrm{~Hz})$, to fast gamma oscillations $(30-80 \mathrm{~Hz})$ and ultra fast ripple oscillations (80 $200 \mathrm{~Hz}$ ) (Buzsaki and Draguhn, 2004). These distinct oscillatory activities correlate with different brain states and in-vivo, oscillations of different frequency ranges can occur in an overlapping fashion. For example, theta oscillations occur during active brain states like exploratory behaviour or navigation in the active animal (Vanderwolf, 1969) and during REM sleep (Jouvet, 1969). In the behaving animal gamma oscillations occur nested within theta oscillations during the same brain states (Bouyer et al., 1981; Fries et al., 2001). Fast ripple oscillations in the hippocampus take place during awake, consummatory behaviour or deep sleep phases (Buzsaki et al., 1992; Buzsaki et al., 1983). Neuronal oscillations are important for the synchronization of the firing pattern of principal neurons across larger regions in the brain and are involved in higher brain function like information retrieval or perception (for review see Bartos et al., 2007; Jensen et al., 2007; Wang, 2010).

\subsubsection{Gamma Oscillations}

Oscillations in the gamma frequency range $(30-80 \mathrm{~Hz})$ are of particular interest, since their occurrence and relation to several higher brain functions has been analysed in detail. Gamma oscillations occur in several brain regions in the behaving animal and are associated with active brains states like exploratory behaviour and REM-sleep phases. They are associated with higher cognitive 
functions like sensory processing and binding of different features, attention to a specific feature and the retrieval and encoding of memory (reviewed by Fries et al., 2007; Jensen et al., 2007; Wang, 2010).

The integration of neuronal activity of distinct brain areas involved in the processing of different features of one object into a coherent picture is mediated by synchronized firing in the gamma frequency band. An inter-area correlation peak in the gamma frequency band between two distinct cortical areas only occurs when features are perceived that activate these distinct brain areas at the same time (Ghazanfar et al., 2008). Therefore, oscillations in the gamma frequency range are important for feature binding and sensory processing in cortical areas (Bauer et al., 2006; Fries, 2009; Ghazanfar et al., 2008; Gray and Singer, 1989). Furthermore, gamma oscillations are correlated with attention states in the brain i.e. the gamma power increases in a certain brain region, when the subject pays attention to a stimulus that activates that particular cortical area (Brovelli et al., 2005; Fan et al., 2007; Tiitinen et al., 1993). Such effects influence the formation of short term and working memory. Enhanced neuronal activity in the gamma range can be recorded in regions activated by a particular stimulus, even after the activating stimulus is gone, which can recruit further cortical regions and might account for the formation of a working, short-term memory (Jokisch and Jensen, 2007; Mainy et al., 2007; TallonBaudry et al., 2005; van Vugt et al., 2010). A disturbance in the generation of gamma oscillations can influence the performance during working memory tasks (Fuchs et al., 2007). Furthermore the retrieval of memory seems to be dependent on gamma oscillations. An increase in gamma power in the hippocampus could be observed in rats during behavioral tasks, where retrieval of previously gained knowledge is necessary (Montgomery and Buzsaki, 2007). These examples show the importance of gamma oscillations on the performance of higher brain functions.

\subsubsection{Generation of Gamma Oscillations}

In order to analyse the mechanisms leading to the generation of gamma oscillations, this neuronal activity was intensively studied in-vitro in the CA3 region of the hippocampus. Gamma oscillations in-vitro can be evoked by different means: electrical stimulation (Whittington et al., 1995), chemical induction with agonists against metabotrophic glutamate receptors (mGluR) (agonist: - (s)-3,5- 
dihydroxyphenylglycine (DHPG)) (Gillies et al., 2002; Whittington et al., 1995), muscarinic acetylcholine receptors (mAChR) (agonist - carbachol) (Fisahn et al., 1998), Kainate receptors (agonist - Kainate) (Fisahn et al., 2004) or by increasing neuronal excitability through the application of potassium-rich solutions (LeBeau et al., 2002). Oscillations induced by Kainate receptor and mGluR agonists are dependent on GABAergic inhibition mediated by $\mathrm{GABA}_{A}$ receptors, because they are blocked by the application of bicuculline. Since they are not inhibited by AMPA receptor antagonists, GABAergic inhibition appears to be both necessary and sufficient to induce gamma oscillations in-vitro (Fisahn et al., 2004; Whittington et al., 1995). On the other hand, oscillations induced by carbachol are blocked by bicucculine and AMPA receptor blockers like NBQX, thus, GABAergic inhibition is necessary but not sufficient for the generation of carbachol-induced oscillations (Fisahn et al., 1998). These findings can be explained by the expression pattern of the respective receptors. Kainate receptors and mGluRs are expressed on interneurons (Fisahn et al., 2004; van Hooft et al., 2000), whereas mAChRs can be found preferentially on pyramidal cells (Fisahn et al., 2002). Furthermore, the existence of gap junctions in interneuron networks is important for the communication within the network and for the generation of oscillatory activity. Gap-junction blockers (Traub et al., 2000) and the knockout of Connexin 36 (Hormuzdi et al., 2001) lead to a reduction of gamma oscillation power.

Generation of gamma oscillations is dependent on a highly connected neuronal network between principal neurons and interneurons, such as the CA3 region of the hippocampus. A high connectivity based on axonal recurrent collaterals and synaptic contacts between principal neurons and interneurons that allows feedforward and feedback inhibition, is necessary for the induction of oscillations (Mann et al., 2005b; for review see Hajos and Paulsen, 2009). In regions, that do not show this feature, gamma oscillations cannot be induced in-vitro. During Kainate application, interneurons are depolarized and start to fire (Fisahn et al., 2004), in a pattern that synchronizes and is transmitted to pyramidal cells. During this process cells that fire out of synchrony are inhibited and are therefore driven to a more synchronized state towards the firing pattern of the other cells. The synchronization process is further supported by the presence of gap junctions. The emerging rhythm of the interneuron network is transmitted to connected pyramidal cells, because they can only fire, when the hyperpolarisation is low (Tiesinga and Sejnowski, 2009), which in turn, excites 
interneurons again (Glickfeld and Scanziani, 2006). This dependence on GABAergic interneurons was also shown by optogenetic experiments, where the enhanced activity of interneurons increases gamma power (Cardin et al., 2009) or induces gamma oscillations (Sohal et al., 2009). Other induction protocols are dependent on an involvement of pyramidal cells in the generation of synchronized firing. Gamma oscillations induced by cholinergic activation are dependent on AMPA mediated excitation of pyramidal cells and interneurons (Fisahn et al., 1998). During these gamma oscillations, pyramidal cells are excited and fire action potentials. Further CA3 pyramidal cells are recruited via recurrent collaterals, which in turn receive inhibitory IPSCs from excited interneurons. Therefore, these cells are inhibited and fire only after the hyperpolarisation fades away in the next gamma cycle (Csicsvari et al., 2003; Mann and Paulsen, 2005; Mann et al., 2005a). Multiple other mechanisms might play a role in generating gamma oscillations in-vivo. During gamma oscillations, interneurons fire synchronized, independent on how this synchronization was achieved, and inhibit principal neurons. Only when this hyperpolarisation fades away, principal cells can fire and the next gamma cycle is initiated (for review see Bartos et al., 2007; Fisahn, 2005; Hajos and Paulsen, 2009; Mann et al., 2005a; Traub et al., 2004).

\subsubsection{Firing Pattern of Distinct Cell Types During Gamma Oscillations}

GABAergic inhibition is crucial for the generation of gamma oscillations (Fisahn et al., 2004). Based on several lines of evidence, the PV-expressing, fast spiking, GABAergic basket cells are especially important for the generation of gamma oscillations. First, this class of cell is abundant in the hippocampus, around $20 \%$ of hippocampal interneurons belong to this group of basket cells (Freund and Buzsaki, 1996). They form highly branched axonal arbors, with intensive contact to other basket cells via chemical and electrical synapses (Fukuda and Kosaka, 2000; Gulyas et al., 1999; Sik et al., 1995). The PV-expressing basket cells also form a highly connected network with pyramidal neurons by innervating the perisomatic region (Freund and Katona, 2007). Therefore, these cells are perfectly positioned to synchronize larger neuronal networks (Cobb et al., 1995). The expression of the outward directed chloride transporter CIC-2 in the postsynaptic cells targeted by PVexpressing basket cells, allows the fast and repetitive stimulation of the postsynaptic 
cell, without a dramatic increase of the chloride concentration in the target cell (Foldy et al., 2010; Smart, 2010). Furthermore, the fast spiking phenotype of PV-expressing basket cells (Jonas et al., 2004) and other intrinsic physiological properties (see 1.3.2) allows them to follow excitatory input faithfully. This feature seems to be crucial for the generation of gamma oscillations. Most importantly, these neurons generate one to two action potentials during each gamma cycle in-vitro (Gloveli et al., 2005; Gulyas et al., 2010; Hajos et al., 2004) and in-vivo (Klausberger et al., 2003; Klausberger et al., 2005; Penttonen et al., 1998; Tukker et al., 2007) and show a high firing activity during these events. Principal neurons do not show this high activity and fire much less frequently during gamma cycles seen in-vitro (Gloveli et al., 2005; Gulyas et al., 2010; Hajos et al., 2004). However, other PV expressing neurons with different morphological features exist. Axo-axonic cells or bistratisfied cells fire also highly phase locked with gamma oscillations (Gloveli et al., 2005; Gulyas et al., 2010; Hajos et al., 2004), whereas O-LM cells fire phase locked to slower frequency ranges (Gloveli et al., 2005; Hajos et al., 2004). Another class of GABAergic interneuron, the trilaminar cell, which sends long projections in the hippocampus, fires highly phase locked to gamma cycles. This might allow them to spread gamma oscillations to other subregions in the hippocampus (Gloveli et al., 2005). Furthermore, based on their perisomatic innervation pattern (see 1.3.2), basket cells expressing CCK could be involved in the generation of gamma oscillations. CCK-positive basket cells are also strongly immunoreactive for CB1-receptors and the fact that application of CB1receptor agonists reduces the power of Kainate induced gamma oscillations in-vitro, suggests that they may be involved in generating gamma oscillations (Hajos et al., 2000). The firing of CCK positive cells themselves is modulated by gamma oscillations (Tukker et al., 2007) and they are involved in the regulation of oscillatory activity in-vivo (Klausberger et al., 2005). However, a direct participation in the generation of gamma oscillations has not been described (Gulyas et al., 2010). The properties of CCK expressing basket cells might position them in a regulatory, finetuning position (Freund and Katona, 2007). In summary, the perisomatic inhibitory PV-expressing basket cells have a crucial role in the generation of gamma oscillations in-vitro and in-vivo. However, other GABAergic interneurons are involved in the generation and regulation of synchronized activity in the brain. 


\subsection{Inhibitory Neurotransmission and Disease}

Inhibitory GABAergic interneurons and neurotransmission play a crucial role in the development of neurons and neuronal circuits. They control the functionality of cortical networks and disturbances in this system may have profound effects on human life resulting in diseases like depression and anxiety (Freund, 2003), neurodevelopmental and psychiatric diseases like schizophrenia (Lewis et al., 2005), autism (Belmonte et al., 2004), Tourette's syndrome (Kalanithi et al., 2005), Rett Syndrome (Chao et al., 2010) or epilepsy (Cossart et al., 2005).

\subsubsection{GABAergic Neurotransmission and Schizophrenia}

Schizophrenia is characterized by psychotic symptoms. Positive symptoms of schizophrenia include the presence of hallucinations (a disturbed perception of external stimuli), delusions (false beliefs) and disorganized thinking; negative symptoms include a reduced emotional expression and poverty of speech. Furthermore, a disturbance in social and occupational life is present. These symptoms have to be present for at least six months and other diseases need to be excluded. Schizophrenia is accompanied by a perturbation in cognitive functions like language, reasoning and planning (for review see: Lewis et al., 2005; Uhlhaas and Singer, 2010). Prominent disturbances in the GABAergic neurotransmitter systems have been described in schizophrenic patients. Reductions of mRNA levels of GAD67 (Akbarian et al., 1995) and for the membrane transporter for GABA, GAT1 (Volk et al., 2001), have been reported. This affects GABA synthesis and re-uptake, respectively, and therefore might cause a decrease in GABAergic neurotransmission. $A$ reduction in expression levels for $P V$, Somatostatin and the $G_{A B A}$-receptor subunits $\alpha 1$ and $\delta$ has also been observed in schizophrenic patients (Akbarian et al., 1995; Hashimoto et al., 2008a; Hashimoto et al., 2008b). The defects in expression of molecules involved in GABAergic neurotransmission are especially observed in PV-expressing neurons (Hashimoto et al., 2003). Furthermore an association of schizophrenia with the activity of NMDA receptors (Belforte et al., 2010; Coyle et al., 2003; Korotkova et al., 2010) has been reported. This influences the GABAergic neurotransmission of GABAergic basket cells, since they receive fast excitatory input via synapses containing NMDR receptors (Belforte et al., 2010; Kinney et al., 2006; 
Korotkova et al., 2010). In total, these alterations in the GABAergic system affect the generation of neuronal oscillations directly. As described above, coordinated rhythmic activity is crucial for higher brain functions like perception, attention and memory processes, which are disturbed in schizophrenic patients. Reduced oscillations in the gamma frequency range have been recorded in schizophrenic patients (Ferrarelli et al., 2008). Furthermore, in schizophrenic patients, the amplitude and phase locking of gamma oscillations to other oscillatory activity is reduced during visual tasks, which points to a disturbed correlation of the respective oscillatory activity in the brain and the processing of the visual stimuli (Uhlhaas et al., 2006). The oscillations occurring during rest in patients are reduced as well, and a disturbed coupling of gamma and theta oscillations could be observed (Koenig et al., 2001). Such deficits in the power and coupling might be the basis for the cognitive deficits seen in schizophrenia patients. Disturbed neuronal oscillations can also be correlated with key symptoms of schizophrenia. For example, a positive correlation between an increase of higher frequency oscillations and a less precise synchronization of different cortical areas has been observed in schizophrenic patients with auditory hallucinations compared to patients without (Ford et al., 2002; Spencer et al., 2009).

Furthermore, the activity of thalamo-cortical networks is involved in the pathophysiology of schizophrenia, as well. The nRT plays an important role in this network, in particular in the gating of sensory information from the thalamus to the cortex (Pinault, 2011). Patients with schizophrenia show a decreased generation of sleep spindles that are generated in the $\mathrm{nRT}$ and these defects are correlated with positive symptoms (Ferrarelli et al., 2007; Ferrarelli et al., 2010). Therefore, perturbations in the $\mathrm{nRT}$ function might be involved in generating symptoms like hallucinations in schizophrenia (Ferrarelli and Tononi, 2011).

\subsubsection{GABAergic Neurotransmission and Epilepsy}

Epilepsy is a term for a diverse group of syndromes with distinct symptoms leading to seizures. An epileptic seizure is caused by hypersynchronized activity in the brain and is based on an imbalance of excitatory and inhibitory neurotransmission (McCormick and Contreras, 2001). Blocking of GABAergic neurotransmission (Dichter and Ayala, 1987) or mutations affecting the GABAergic system lead to epileptic discharges (Noebels, 2003). Epileptic discharges are 
characterized by either low frequency spike-wave discharges (absence seizures) or large-amplitude oscillations measured by EEG. Increased excitation among principal neurons in the brain and/or a disturbed GABAergic inhibition can cause these large discharges. These epileptic discharges can propagate through neuronal networks and influence further brain areas (McCormick and Contreras, 2001).

Epileptic seizures have profound consequences for neuronal networks. Seizures generated in the hippocampus cause extensive reorganization in the CA3 region with sprouting of axons of dentate gyrus granule cells (mossy fibre sprouting) (Represa et al., 1987) and formation of new functional synapses (neosynapses) (Frotscher and Zimmer, 1983; for review see: Ben-Ari, 2001; Morimoto et al., 2004). The newly formed synapses are functional but aberrantly positioned (Represa et al., 1993a; Represa et al., 1993b). This newly acquired plasticity is also observed in the CA1 region of the hippocampus (Esclapez et al., 1999). The consequences are an enhanced excitability and an increase in the susceptibility of the newly formed network to further seizures - "seizure begets seizure" (Ben-Ari et al., 2007). This has been shown in the in the mature hippocampus (kindling model) (for review see: Morimoto et al., 2004) but also in the developing hippocampus. Seizures in one hippocampus propagate to the other hemisphere and repeated epileptic discharges in one hippocampus can induce this activity in the other, naive side. This newly acquired activity is persistent and the transformation is dependent on GABAergic neurotransmission and the presence on high frequency oscillations in the hippocampal network (Khalilov et al., 2003; Khalilov et al., 1997; Khalilov et al., 2005).

Increased excitation and disturbed GABAergic neurotransmission is important for the formation of epileptic discharges. However, GABA signalling is more complex. It does not function in a purely inhibitory fashion. GABA mediated chloride currents can also lead to an increase in membrane conductance (shunting) and depolarization. Thus, GABA can lead to increased excitability even in adult neurons (Chavas and Marty, 2003; Vida et al., 2006). GABAergic interneurons are involved in the synchronization of neuronal networks. Furthermore, a high diversity of GABAergic neurons that are incorporated in different networks to perform different tasks is present in the brain. Disturbances at all these levels can be involved in the generation of an epileptic phenotype (Cossart et al., 2005). The composition of GABA receptors is changed after status epilepticus in human patients (Loup et al., 
2000) and animal models (Bouilleret et al., 2000; Schwarzer et al., 1997). Such postsynaptic alterations influence the sensitivity to GABA and other modulators (Gibbs et al., 1997). Seizures lead to an inhibitory-to-excitatory switch of GABAergic neurotransmission in cells (Cohen et al., 2002; Khalilov et al., 2003; Khalilov et al., 2005), which may be caused by a loss of KCC2 leading to an increased intracellular chloride concentration (Ben-Ari, 2008b). Therefore, epilepsy leads to a return to immature properties in the epileptic tissue. Loss of somatostatin expressing GABAergic neurons is obvious in epileptic tissue (Buckmaster and Jongen-Relo, 1999; Mathern et al., 1995), whereas other classes of interneurons are less vulnerable, including PV-expressing neurons (van Vliet et al., 2004). Dendrite targeting, somatostatin positive O-LM cells are lost, which leads to a decreased inhibition of distal dendrites of pyramidal neurons (Cossart et al., 2001). This loss might be directly implicated in the enhanced seizure susceptibility of the reorganized network (Wendling et al., 2002). On the other hand, the perisomatic innervation pattern of PV expressing cells is preserved in the epileptic hippocampus (Wittner et al., 2005; Wittner et al., 2001). Epilepsy shows a prominent disturbance in GABAergic signalling that is more diverse than just a simple lack of inhibition.

\subsection{Aim of the Study}

A conventional knockout of Viaat shuts down GABA and glycine release from inhibitory neurons. These mice die before birth, which prevents the analysis of the postnatal GABAergic and glycinergic neurotransmitter systems and of their contribution to network function and the development of neuronal circuits. Therefore, a combination of a conditional Viaat allele flanked by two loxP-sites with the transgenic expression of Cre-recombinase under the control of the PV and VGLUT3 promoter was used to silence distinct subsets of neurons. This allows the analysis of the contribution of GABAergic neurotransmission to the morphological development of neurons and neuronal circuits in the postnatal brain. Furthermore, the contribution of two distinct subsets of GABAergic neurons to circuit function could be analysed in more detail.

Morphological analysis of biocytin-filled neurons was used to analyse the contribution of GABAergic neurotransmission to the development of single neurons in $V_{\text {Viaat }}{ }^{\mathrm{f} / \mathrm{fl}}$, PV-Cre animals. The functional consequences of deleting Viaat in PV- 
positive and in VGLUT3-positive interneurons, which both provide perisomatic inhibition to pyramidal neurons in the hippocampus were compared by analysing Kainate induced gamma oscillations in-vitro.

In this study, the contribution of GABAergic neurotransmission to the morphological development of neurons and of neuronal circuits was analysed. At the functional level, this study directly compares the involvement of two distinct neuron types with different physiological properties, but similar morphological features in the generation of gamma oscillations, a network activity critical to higher brain functions. 


\section{Materials and Methods}

\subsection{General Materials}

The following table contains the general chemicals and kits used in this study:

\begin{tabular}{|c|c|}
\hline ABC-reagent & Vector Laboratory Inc \\
\hline Acrylamid/bisacrylamid & Bio-Rad Laboratories GmbH \\
\hline APS (Ammoniumpersulfate) & Sigma-Aldrich \\
\hline AquaPolyMount & Polyscience Inc \\
\hline ATP- $\mathrm{Mg}^{2+}$ & Sigma-Aldrich \\
\hline B27 & Gibco - Invitrogen \\
\hline BCA-kit & Thermo Scientific \\
\hline Biocytin hydrochloride & Sigma-Aldrich \\
\hline BSA & $\begin{array}{l}\text { Sigma-Aldrich or Biomol or Thermo } \\
\text { Scientific }\end{array}$ \\
\hline Corn Oil & Sigma-Aldrich \\
\hline DAB-kit & Dako \\
\hline Developer & Agfa \\
\hline DMEM & Dibco - Invitrogen \\
\hline ECL detection reagent & $\begin{array}{l}\text { Amersham }{ }^{\mathrm{TM}} \text { ECL Western blotting } \\
\text { detection reagents - GE healthcare }\end{array}$ \\
\hline Fetal Calf Serum & PAA Laboratories $\mathrm{GmbH}$ \\
\hline FluormoutG & Southern Biotech \\
\hline Gelatin & Riedel-de Haën \\
\hline GlutaMAX TM 1 & Gibco - Invitrogen \\
\hline Glutaraldehyd & $\begin{array}{l}\text { Merck KGaA, Electron Microscopy } \\
\text { Science }\end{array}$ \\
\hline Glycerol & Sigma-Aldrich \\
\hline Goat serum & PAA Laboratories $\mathrm{GmbH}$ \\
\hline Goldchlorid & Sigma-Aldrich \\
\hline GTP-Na ${ }^{+}$ & Sigma-Aldrich \\
\hline $\mathrm{H}_{2} \mathrm{O}_{2}$ & Merck KGaA \\
\hline HBSS & Gibco - Invitrogen \\
\hline Isoflurane & DeltaSelect \\
\hline Isopentane/Isomethylbutane & Roth \\
\hline Kainic Acid & Sigma-Aldrich \\
\hline Ketamine & Cp pharma \\
\hline L-Cystein & Sigma-Aldrich \\
\hline Milk Powder & Frema \\
\hline Natriumazid & Merck KGaA \\
\hline NBQX disodium salt & Tocris Bioscience \\
\hline Neurobasal-A & Gibco - Invitrogen \\
\hline Papain & Worthington Biomedical Corporation \\
\hline Paraformaldhyde & SERVA Electrophoresis GmbH \\
\hline Penicillin/Streptomycin & Gibco - Invitrogen \\
\hline Poly-L-lysine & Sigma-Aldrich \\
\hline Ponceau-S & Sigma-Aldrich \\
\hline Protease inhibitor & Roche Diagnostics $\mathrm{GmbH}$ \\
\hline
\end{tabular}




\begin{tabular}{|l|l|}
\hline Rapid fixer & Agfa \\
\hline Salts for electrophysiology & Merck KGaA \\
\hline Silvernitrate & Merck KGaA \\
\hline Sodiumthiosulfate & Merck KGaA \\
\hline Sucrose & $\begin{array}{l}\text { Sigma (pure for Electrophysiology), } \\
\text { Merck KGaA }\end{array}$ \\
\hline Tamoxifen & Sigma-Aldrich \\
\hline TEMED & Serva Electrophoresis GmbH \\
\hline Thioglycolic acid & Sigma-Aldrich \\
\hline Tribromethanol & Sigma-Aldrich \\
\hline Triton-x-100 & Roche Diagnostics GmbH \\
\hline Trypsin inhibitor & Sigma-Aldrich \\
\hline Trypsin/EDTA & Gibco- Invitrogen \\
\hline TTX & Tocris Bioscience \\
\hline Tween-20 & Sigma-Aldrich \\
\hline Xylazine & Cp pharma \\
\hline
\end{tabular}

Table 2.1: General Chemicals Used in this Study

This table contains general material used in this study:

\begin{tabular}{|l|l|}
\hline Blotting paper (Whatman-3MM Chr) & Whatman International LId \\
\hline ECL films & $\begin{array}{l}\text { Amersham Hyperfilm ECL }- \text { GE } \\
\text { healthcare }\end{array}$ \\
\hline Glass capillaries & Science Products \\
\hline Glass coverslips & Menzel Gläser \\
\hline $\begin{array}{l}\text { Plastic ware (reaction tubes, tubes, well- } \\
\text { plates) }\end{array}$ & General Laboratory Supplier \\
\hline Superfrost glass slides & Thermo Scientific, Menzel Gläser \\
\hline
\end{tabular}

Table 2.2: General Material Used in this Study

\subsection{Mouse Lines}

Animals of an age between postnatal day 18 and 22 were used for experiments analysing mutant animals. For the analysis of CreERT2 mouse lines also older animals of around $6-10$ weeks of age were used. This was in agreement with the guidelines for the welfare of experimental animals from the Federal Government of Germany and the Max Planck Society.

\subsubsection{Viaat Conditional Knockout}

The mouse line containing a conditional floxed Viaat allele was designed and generated by Dr. S. Wojcik. An 11.2kb BamHI/Sbfl fragment containing the Viaat 
gene was subcloned from Cosmid MPGc121F07737Q2 into pSKII. Subsequently, a thymidine kinase cassette for negative selection was cloned into the Spel site of pSKII. LoxP sites flanking the second exon and a neomycin selection cassette flanked by Frt sites were introduced in two recombineering steps in E.coli as described in (Liu et al., 2003) using the $\lambda$ Red system of EL350 and SW105 (http://web.ncifcrf.gov/research/brb/productDataSheets/recombineering/bacterialStrai ns.aspx). The correct integration of the loxP sites was confirmed by restriction digests and sequencing.

The linearised targeting vector was electroporated into 129/Ola ES-cells and clones screened by Southern blot. Clones with the correct homologous recombination were injected into C57BL/6 blastocysts by experienced staff of the animal facility of the institute. Male chimeras were crossed to C57BL/6 females to check for germline transmission. The neomycin cassette was removed by breeding males carrying the targeted Viaat allele to C57BL/6 females expressing FlpRecombinase (TgN(ACTFLPe)9205Dym, from Jackson Laboratory).

\subsubsection{Transgenic Animals}

\subsubsection{Parvalbumin Cre-transgenic Mice}

The mouse line expressing Cre-recombinase under the control of the Parvalbumin (PV) promoter was generously provided by Prof. H. Monyer. The generation of the transgenic line was described elsewhere (Fuchs et al., 2007; Monyer and Markram, 2004).

\subsubsection{Other Cre-transgenic Mice}

Cre-transgenic mouse lines expressing the recombinase under the control of the promoters for Calbindin (CB), Calretinin (CR) and VGLUT3 were designed and generated by Dr. S. Wojcik. A tamoxifen inducible form of Cre, CreERT2 (Erdmann et al., 2007; Feil et al., 1997), was used to generate transgenic animals for all three promoters. Additionally, transgenic animals expressing the conventional form of Cre under the control of the VGLUT3 promoter were generated. All mouse lines were designed as bacterial artificial chromosome (BAC) transgenic animals. CB-CreERT2, 
CR-CreERT2, VGLUT3-CreERT2 and VGLUT3-Cre BACs were developed by homologous recombination in E.coli (Liu et al., 2003; Yang et al., 1997). After successful generation and purification of the BAC-constructs, the linearised DNA was injected into the pronuclei of fertilized eggs by experienced staff of the animal facility of the institute. Integration of the BAC transgenes in founder animals was confirmed by PCR, and transgene expression was assessed by breeding founder animals or their offspring to animals carrying the CAG-CAT-EGFP Cre-reporter transgene. Plasmids containing Cre and CreERT2, which were used for the generation of the BAC-transgenes were generously provided by Dr. Stefan Berger and Prof. Günther Schütz (Erdmann et al., 2007).

\subsubsection{CAG-CAT-EGFP Reporter Mouse Line}

This transgenic mouse line was used to visualize Cre-expression in the conditional knockout animals and the Cre-transgenic mouse lines. The generation of the mouse line is described elsewhere (Nakamura et al., 2006).

\subsection{Genotyping of Animals}

The genotyping of DNA from tail biopsies was performed by the DNA core facility of the MPI-em.

\subsection{Tamoxifen Treatment of CreERT2-mouse Lines}

Tamoxifen was dissolved in corn oil at a concentration of $10 \mathrm{mg} / \mathrm{ml}$ under vigorous agitation. The solution was sterile filtered and transferred into the animal facility of the institute. 100mg Tamoxifen/kg bodyweight/ day were injected intraperitoneally once per day for 10 consecutive days or divided into two doses twice per day for 5 consecutive days. After 10 days the animals were analysed for expression of the CreERT2 transgene. 


\subsection{Immunofluorescence Analysis of Brain Sections}

Mice were deeply anaesthetised by intraperitoneal injection of $2 \%$ tribromethanol $(4.5 \mathrm{ml}$ saline $+0.4 \mathrm{ml}$ ethanol pure $+0.1 \mathrm{ml}$ TBE stock, use around $300-400 \mu \mathrm{l}$ of dilution for adult mice, around $500-600 \mathrm{mg} / \mathrm{kg}$ body weight; stock $=1.6 \mathrm{~g}$ $\mathrm{TBE} / \mathrm{ml}$ tert-amyl alcohol) and subsequently transcardially perfused with a flush of HBSS, followed by $4 \%$ paraformaldehyd (PFA) in $0.1 \mathrm{M}$ PB-buffer for $10 \mathrm{~min}$. The brain was dissected, postfixed for one hour in 4\% PFA in $0.1 \%$ PB on ice and cryoprotected in a series of $10 \%, 20 \%$ and $30 \%$ sucrose solutions in PB-buffer at $4^{\circ} \mathrm{C}$. Alternatively, for co-labelling of Viaat and Gephyrin in the cerebellum, the animals were perfused with 4\% PFA $+0.1 \%$ Glutaraldehyd for $5 \mathrm{~min}$. After dissection, the brain was postfixed for $10 \mathrm{~min}$ in $4 \%$ PFA on ice, briefly washed two times in $0.1 \mathrm{M}$ PB followed by cryoprotection in $30 \%$ sucrose overnight at $4{ }^{\circ} \mathrm{C}$.

Brains were frozen at $-35^{\circ} \mathrm{C}$ in 2-methylbutane and $40 \mu \mathrm{m}$ coronal brain sections were cut in a cryostat (Leica) in $0.1 \mathrm{M}$ PB-buffer $+0.02 \%$ sodiumazide and further processed for immunofluorescence staining. The sections were briefly washed in PBbuffer, blocked and permeabilized for 1 hour in 5\% goat-serum and $0.3 \%$ Triton-X100 in $0.1 \mathrm{M}$ PB. Thereafter, sections were incubated in primary antibody for 48 hours at $4^{\circ} \mathrm{C}$ in $2 \%$ goat-serum and $0.3 \%$ Triton-X-100 in $0.1 \mathrm{M} \mathrm{PB}$. After several wash steps, the sections were treated with fluorophore-coupled secondary antibody for $1 \mathrm{~h}$ at RT in 5\% goat-serum and $0.3 \%$ Trition-X-100 in $0.1 \mathrm{M}$ PB. After washing, the sections were embedded in FluoromountG or AquaPolyMount on Superfrost glass slides and stored at $4^{\circ} \mathrm{C}$ for analysis.

For the analysis of Cre-mouse lines and for counting of PV-positive cells the mice were treated as above (perfusion with 4\% PFA, 1 h postfixation, 10\%, $20 \%, 30 \%$ sucrose). After cryoprotection the brain was embedded in a gelatin block ( $7 \%$ gelatin, $15 \%$ sucrose, $0.02 \% \mathrm{NaAzide}$ in $1 \mathrm{x} \mathrm{PBS}$ ) and frozen at $-35^{\circ} \mathrm{C}$ in 2-methylbutane. The block was stored at $-80^{\circ} \mathrm{C}$ until further processing. Sections of $14 \mu \mathrm{m}$ thickness were cut in a cryostat and thaw-mounted on Superfrost glass slides. During the processing and collecting, the glass slides were kept in the cryostat and further stored at $-80^{\circ} \mathrm{C}$ without any thawing of the sections in between. For immunofluorescence labelling, the sections were thawed at RT and washed in 0.1M PB. The sections were blocked for $30 \mathrm{~min}$ in $5 \%$ goat serum, $5 \%$ sucrose, $0.3 \%$ Triton X-100 in PBS at RT. Primary antibody incubation was performed overnight at $4^{\circ} \mathrm{C}$ in $3 \%$ goat serum, $5 \%$ sucrose, 
$0.3 \%$ Triton-X-100 in PBS. After washing the sections were treated with a fluorophore-coupled secondary antibody diluted in blocking solution for $1 \mathrm{~h}$ at RT in the dark. After washing, the glass slides were embedded with FluormountG, dried and stored at $4^{\circ} \mathrm{C}$ until analysis.

\subsection{Immunofluorescence Analysis of Cultured Primary Mouse Neurons}

Culture medium was removed, cells quickly washed with $1 \mathrm{XPBS}\left(-\mathrm{K}^{+}\right)$and immediately fixed for 20 min with $4 \%$ PFA + glucose $(120 \mathrm{mM})$ in 1XPBS. Cells were blocked for $30 \mathrm{~min}$ at RT with blocking buffer (10\% Goat-serum, 2\% BSA, 5\% sucrose, $0.3 \%$ Triton-X-100 in 1 XPBS). The primary antibody was diluted in blocking buffer and incubated at $4^{\circ} \mathrm{C}$ overnight. Coverslips were washed $4 \times 5 \mathrm{~min}$ with wash buffer (1xPBS, 0.3\% Triton-X-100) and fluorophore coupled secondary antibody diluted in the same buffer. Cells were incubated for $1 \mathrm{~h}$ at RT in the dark. Coverslips were washed with wash buffer and during the last step with PBS. Coverslips were embedded with FluoromountG on Superfrost Glass slides, dried and stored at $4^{\circ} \mathrm{C}$ until imaging.

\subsection{Antibodies}

\subsubsection{Primary Antibodies}

The following table lists the primary antibodies used in this study:

\begin{tabular}{|l|l|l|l|l|l|}
\hline Antigen & Clone & Company & Host & IHC & Western blot \\
\hline \multirow{3}{*}{ Calbindin } & & Swant & Mouse & $1: 2000$ & \\
\cline { 2 - 6 } & & $\begin{array}{l}\text { Chemicon/ } \\
\text { Millipore }\end{array}$ & Rabbit & $1: 3000$ & \\
\hline \multirow{2}{*}{ Calretinin } & & Swant & Mouse & $1: 4000$ & \\
\cline { 2 - 6 } & & $\begin{array}{l}\text { Chemicon/ } \\
\text { Millipore }\end{array}$ & Rabbit & $1: 2000$ & \\
\hline \multirow{2}{*}{ EGFP } & & Millipore & Mouse & $1: 700$ & \\
\cline { 2 - 6 } & & Invitrogen & Rabbit & $1: 5000$ & \\
\hline \multirow{2}{*}{ GAD65 } & GAD-6 & $\begin{array}{l}\text { Chemicon/ } \\
\text { Millipore }\end{array}$ & Mouse & $1: 1500$ & \\
\hline
\end{tabular}




\begin{tabular}{|l|l|l|l|l|l|}
\hline GAD67 & $1 \mathrm{G} 10.2$ & $\begin{array}{l}\text { Chemicon/ } \\
\text { Millipore }\end{array}$ & Mouse & $1: 2000$ & \\
\hline Gephyrin & $3 \mathrm{~B} 11$ & Synaptic Systems & Mouse & $1: 1000$ & $1: 20,000$ \\
\hline \multirow{2}{*}{ Parvalbumin } & & Swant & Mouse & $1: 4000$ & \\
\cline { 2 - 6 } & & Swant & Rabbit & $1: 5000$ & \\
\hline Rim1 $\alpha$ & & $\begin{array}{l}\text { Transduction } \\
\text { Laboratories }\end{array}$ & Mouse & & $1: 500$ \\
\hline Synapsin 1+2 & & Synaptic Systems & Rabbit & $1: 2000$ & \\
\hline Synaptophysin & 7.2 & Synaptic Systems & Mouse & & $1: 20,000$ \\
\hline \multirow{2}{*}{ VGAT/Viaat } & & Synaptic Systems & Rabbit & $1: 1000$ & $1: 1000$ \\
\cline { 2 - 6 } & & Chemicon & Rabbit & & $1: 1000$ \\
\hline VGLUT3 & & Synaptic Systems & Rabbit & $1: 1000$ & \\
\hline
\end{tabular}

Table 2.3: Primary Antibodies Used in the Study

The antigen refers to the protein, against which the antibody is directed. Clone refers to the designated number of monoclonally produced antibodies. Company describes the commercial supplier of the antibody. Host describes the source of the antibody, in which the antibody was produced. Mouse refers to monoclonal antibodies. The last two columns describe the used working dilutions of the antibody in immunohistochemistry (IHC) or in western blot analysis.

\subsubsection{Secondary Antibodies}

The following table summarizes the secondary antibodies used in this study:

\begin{tabular}{|l|l|l|l|}
\hline Antibody & Coupled to: & Dilution & Company \\
\hline Goat-anti mouse & Alexa 488, 555 or 633 & $1: 1200$ & Molecular Probes \\
\hline Goat-anti rabbit & Alexa 488, 555 or 633 & $1: 1200$ & Molecular Probes \\
\hline $\begin{array}{l}\text { Goat-anti Mouse } \\
\text { (affinity purified) }\end{array}$ & horseradish peroxidase & $1: 10,000$ & $\begin{array}{l}\text { Jackson } \\
\text { ImmunoResearch } \\
\text { Laboratories }\end{array}$ \\
\hline $\begin{array}{l}\text { Goat-anti Rabbit } \\
\text { (affinity purified) }\end{array}$ & Horseradish peroxidase & $1: 10,000$ & $\begin{array}{l}\text { Jackson } \\
\text { ImmunoResearch } \\
\text { Laboratories }\end{array}$ \\
\hline
\end{tabular}

Table 2.4: Secondary Antibodies Used in this Study

\subsection{Imaging and Picture Analysis}

Brain sections of $40 \mu \mathrm{m}$ thickness were analysed on a confocal microscope. Single-plane confocal images were taken on an inverted TCS-SP2 confocal laserscanning microscope (Leica Microsystems) with a 63x (NA 1.4) oil-immersion 
objective lens with a digital zoom factor of 2 or 4 for quantification. During the acquisition, gain and offset were kept constant in order to allow comparison between mutant and control animals. Confocal images were further analysed with AnalySIS software (Olympus). Original images were binarised by standard thresholding. A particle separator was applied to the binarised picture and puncta above a certain threshold were counted in the Region Of Interest (ROI, whole image or single cells for Gephyrin quantification). Images were additionally analysed manually.

To screen the Cre-transgenic mice for expression of Cre and CreERT2, $14 \mu \mathrm{m}$ brain sections were imaged with an upright, epifluorescent microscope (Olympus BX61) equipped with a 10x or 20x objective and the F-View Soft Imaging System (Olympus) camera coupled to the AnalySIS software. Additional images were taken with a Zeiss Apotome AX10 Imager equipped with a 25x oil immersion objective.

\subsection{Processing of $300 \mu m$ Vibratome Brain Sections}

To visualize neurons that were filled with Biocytin during electrophysiological recordings, the slice was left in the recording chamber for recovery for $15 \mathrm{~min}$. After the recovery, slices were transferred to $4 \% \mathrm{PFA}$ in $0.1 \mathrm{M} \mathrm{PB}$ and fixed overnight at $4^{\circ} \mathrm{C}$. The sections were then thoroughly washed with $0.1 \mathrm{M} \mathrm{PB}$ and cryoprotected overnight at $4{ }^{\circ} \mathrm{C}$ in cryoprotection media (25\% Sucrose, $10 \%$ Glycerol in $0.1 \mathrm{M} \mathrm{PB} \mathrm{pH}$ 7.4). Slices were permeabilized by three freezing-thawing steps over liquid nitrogen. After washing, endogenous peroxidase activity was quenched with $1 \% \mathrm{H}_{2} \mathrm{O}_{2}$ in $0.1 \mathrm{M}$ PB for 10min. After extensive washing in 0.1M PB, 0.05M TB pH7.6 and 0.05M TBS $(0.05 \mathrm{M} \mathrm{TB}+0.9 \% \mathrm{NaCl})$, the sections were incubated overnight in a 1:400 dilution of $A B C$ reagent in $0.05 \mathrm{M}$ TBS at $4^{\circ} \mathrm{C}$. After extensive washing, the sections were developed with DAB solutions according to the manufacturer's protocol. Sections were incubated under visual control for around $15 \mathrm{~min}$. The reaction was stopped by extensive wash steps in TB and PB. Sections were stored in PB-buffer until further processing.

Subsequently, the DAB staining was intensified with silver impregnation. The sections were washed briefly with distilled water and incubated with $5 \%$ Thioglycolic acid for $1 \mathrm{~h}$ at RT. The sections were treated with $1.42 \%$ Silver nitrate for $30 \mathrm{~min}$ at $56^{\circ} \mathrm{C}$, dark, followed by an incubation with $0.2 \%$ Gold chloride for 10 min at RT, dark. After this, the sections were treated with $5 \%$ Sodium thiosulphate solution. After 
every reaction step the sections were washed with distilled water for $10 \mathrm{~min}$. The last washing step was with $0.1 \mathrm{M}$ PB for $2 \times 5 \mathrm{~min}$ and one overnight wash at $4^{\circ} \mathrm{C}$ in $\mathrm{PB}$. The sections were mounted on Superfrost glass slides, embedded with AquaPolyMount and stored at $4^{\circ} \mathrm{C}$ until further processing.

\subsection{Analysis of DAB-stained Sections}

The cells were scanned with a Zeiss Imager.M2 AX10 (Apotome) with an 25x water immersion objective (Zeiss Plano Apochromat, $\mathrm{n}$ 0.8) connected to a mbf Biosciences $Q$ imaging camera and controlled with the Neuroleucida software (mbf Bioscience, Version 10). The area to be scanned was defined under low power magnification (10x dry objective, EC Plan Neoflura $n=0.3$ ). One $\mu \mathrm{m}$ thick optical sections were taken to cover the complete cell soma, dendritic and axonal tree. Reconstruction of the somatodentritic region of filled $\mathrm{nRT}$ cells was performed with Neurolucida software and key parameters for analysis were obtained with the same software programme.

\subsection{Primary Mouse Neuron Culture}

\subsubsection{Continental Primary Mouse Neuron Cultures - Treatment of Coverslips}

To obtain a direct adhesion of the neurons to the glass coverslip, the surface was coated with poly-L-lysine. A solution of $1 \mathrm{ml}$ poly-L-Lysine $(0.01 \%)$ in $11 \mathrm{ml}$ sterile PBS was incubated for one hour or overnight at $37^{\circ} \mathrm{C}$ on the coverslips. Wells were washed three times with sterile HBSS under sterile conditions and then incubated with complete Neurobasal-A-medium until use.

\subsubsection{Astrocyte Microisland Culture for Primary Mouse Neuronal Autaptic Cultures}

Autaptic neurons are cultured on astrocyte feeder cells in order to provide optimal growth conditions for the nerve cells. Astrocytes are seeded on microdots of 
permissive substrate on glass coverslips. The astrocyte microisland plates were provided by technical service in the laboratory.

Briefly, $0.15 \%$ agarose was dissolved, pipetted onto the glass coverslips and the coverslips sterilized by a 20-30min UV-light treatment. A mixture of Poly-DLysine/ Collagen / acetic acid (1:1:3) was stamped on the agarose surface with a custom-made stamp in order to create dots of permissive substrate (collagen)

Mouse cortices of newborn wild type animals were dissected and digested with Trypsin/EDTA $(0.05 \% / 1 \mathrm{x}, \mathrm{Gibco})$ at $37^{\circ} \mathrm{C}$ with gentle agitation. The supernatant was removed and the reaction stopped by applying FCS-medium (10\% FCS, MITO, Pen/Strep in DMEM). Digested tissue was triturated in $0.2 \mathrm{ml}$ of pre-warmed FBSmedium and cells transferred in fresh medium. Cells were counted and seeded (800.000 cells) into a $75 \mathrm{~cm}^{2}$ culture flask, grown to confluence and re-seeded in prepared 6-well plates after trypsin treatment (density 12.000 cells/6-well)

\subsubsection{Primary Mouse Striatum Neuron Culture}

\subsubsection{Solution and Media}

Solution 1:

2mg Cystein, 10ml DMEM, $1 \mathrm{mM} \mathrm{CaCl}_{2}$ (stock 100mM), 0.5mM EDTA (stock $50 \mathrm{mM}$ ), stored at $-20^{\circ} \mathrm{C}$ in $5 \mathrm{ml}$ aliquots, pre-warm to $37^{\circ} \mathrm{C}$

Papain solution:

25 units Papain/ml in solution 1 mixed and incubated with carbogen gas (95\% $\mathrm{O}_{2}, 5 \% \mathrm{CO}_{2}$ ), for $25 \mathrm{~min}$, stored on ice and sterilized by filtration through a $0.2 \mathrm{~mm}$ filter before use.

Stop Solution:

25mg Albumin, 25mg Trypsin Inhibitor, 9ml DMEM, 1ml FCS. The solution was prewarmed and kept at $37^{\circ} \mathrm{C}$ until use.

Neurobasal-A-Medium:

100ml Neurobasal-A, 2ml 1xB27 supplement, 1ml Glutamax, 0.5ml Pen/Strep. Medium was sterilized by filtration and stored at $4^{\circ} \mathrm{C}$ 


\subsubsection{Preparation of Mouse Neuron Culture}

Brains from newborn homozygously floxed (Viaat ${ }^{f / f l}$ ) animals were collected in HBSS at RT. The striatum was dissected and transferred immediately to $0.8 \mathrm{ml}$ Papain solution and kept on ice until all tissue was dissected. Tissue was digested for $55 \mathrm{~min}$ at $37^{\circ} \mathrm{C}$ under gentle agitation. The supernatant was carefully removed without disturbing the tissue. To stop the Papain digest, $0.8 \mathrm{ml}$ pre-warmed stopsolution was added and incubated for $15 \mathrm{~min}$ at $37^{\circ} \mathrm{C}$ under slight agitation. The solution was discarded and around $120 \mu$ l pre-warmed Neurobasal-A-medium was added. The tissue was gently triturated around 10 times with a yellow tip and $100 \mu l$ cell-suspension-supernatant was transferred in $700 \mu \mathrm{l}$ pre-warmed neurobasal-Amedium. The trituration process was repeated for a total of 3 times with fresh medium. Cells were counted with a Neubauer Counting chamber and cells were seeded into Neurobasal-A-Medium as following: around 6,500 cells on Astrocytemicroisland plates, between 30,000 and 45,000 cells on glass coverslips in 24-wellplates and around 700,000 - 750,000 cells in treated $6 \mathrm{~cm}$ dishes. The cells were incubated at $37^{\circ} \mathrm{C}$ for 14 days to 3 weeks before analysis.

Neurons were infected with Cre-EGFP-Lentivirus or EGFP-Lentivirus (provided by Dr. H. Kawabe) at day in-vitro (DIV) 1 under S2 working conditions and the cultures were used for biochemical, cell biological or electrophysiological experiments between DIV12 and DIV20.

\subsection{Biochemical Assays}

\subsubsection{Preparation of Whole Brain Homogenate}

Homogenisation buffer (320mM sucrose, 5mM HEPES pH 7.4, 0.1mM EDTA, 1:1000 dilution of protease inhibitor PMSF, Leupeptin and Apoleptin), $15 \mathrm{ml}$ glass potter and all plastic ware were cooled down on ice. The dissected brain was placed in ice-cold homogenization buffer and homogenized at 900rpm with a glass-Teflonhomogenizer (B Braun Biotech). The homogenized sample was aliquoted and quick frozen in liquid nitrogen. The protein concentration was determined using the BCAmethod (Pierce) according to manufacturer's instructions. The density values were linearly correlated with concentrations of a standard curve and the concentrations of the samples were calculated. The protein concentration was adjusted to $2 \mu \mathrm{g} / \mu \mathrm{l}$ with 
$3 x$ Laemli buffer. Samples were heated for $3 \mathrm{~min}$ at $95^{\circ} \mathrm{C}$ and stored at $-80^{\circ} \mathrm{C}$ until use.

\subsubsection{Preparation of Lysates of Cultured Neurons}

Densely grown cultures of mouse striatal neurons were washed 3 times with ice-cold PBS and lysed by application of $150 \mu$ lysis buffer $(320 \mathrm{mM}$ Sucrose, $10 \mathrm{mM}$ Tris/HCl pH7.5, 5mM HEPES pH 7.4, 150mM NaCl, 1mM EDTA, 1mM EGTA, 0.1\% IGEPAL) on ice. With the help of a cell scraper, the cells were efficiently removed from the glass coverslips or plastic bottom. The lysate was centrifuged at 900xg for 10 minutes and the protein concentration of the supernatant determined using the BCA-method. The protein concentration was adjusted to around $0.5 \mu \mathrm{g} / \mu \mathrm{l}$ with $3 x$ Laemli buffer and samples were stored at $-80^{\circ} \mathrm{C}$ for Western Blot analysis.

\subsubsection{Sodium-Dodecyl-Sulfate-Polyacrylamid-Gel Electrophoresis (SDS-PAGE)}

Proteins are separated based on their molecular weight during migration through a porous gel matrix, the pore size is determined by the concentration of Polyacrylamid in the gel.

SDS-Gels consists of a separating gel, in which the proteins are separated and a stacking gel, where the proteins are concentrated in a thin layer before migrating into the separating gel.

\subsubsection{Solutions}

Separating gel buffer:

1.5M Tris/Cl, $0.4 \%$ SDS, $\mathrm{pH} 8.8$

Stacking gel buffer:

$0.5 \mathrm{M}$ Tris/Cl, $0.4 \%$ SDS, $\mathrm{pH} 6.8$

Acrylamid-solution:

$30 \%$ Acrylamid/N,N'-Methylene-bis-Acrylamid Solution 
10\% APS:

$10 \%$ Ammoniumpersulfat in $\mathrm{H} 20$

TEMED-solution:

N,N,N',N'-tetramethylethylenediamine solution

Running Buffer:

25mM Tris Base, 190mM Glycine, 1\% SDS, pH 8.8

\subsubsection{Preparing Gels and Separating Proteins}

The following table summarizes the composition of SDS-gels:

\begin{tabular}{|l|l|l|}
\hline & Separating Gel (3 gels) & Stacking gel (3 gels) \\
\hline Acrylamid solution & $10 \mathrm{ml}(15 \%$ gel $)$ & $2.5 \mathrm{ml}(7.5 \%$ gel $)$ \\
\hline Buffer & $5 \mathrm{ml}$ & $2.5 \mathrm{ml}$ \\
\hline $\mathrm{H} 2 \mathrm{O}$ & $4.8 \mathrm{ml}$ & $4.9 \mathrm{ml}$ \\
\hline $10 \%$ APS & $125 \mu \mathrm{l}$ & $62.5 \mu \mathrm{l}$ \\
\hline TEMED & $25 \mu \mathrm{l}$ & $12.5 \mu \mathrm{l}$ \\
\hline
\end{tabular}

\section{Table 2.5: Composition of Separating and Stacking Gel for SDS-PAGE}

The separating gel solution was poured into the assembled gel chamber and the surface was overlaid with water-saturated butanol in order to obtain a smooth surface. After the polymerization the butanol was discarded, the stacking gel poured on top of the separating gel, and the comb of the desired size was inserted to obtain wells for loading. After complete polymerization of the gel, around $20 \mu \mathrm{g}$ of heated brain homogenate sample in $1 \mathrm{x}$ Laemmli buffer was loaded onto the gel and the electrophoresis took place with $10 \mathrm{~mA}$ per gel for around $2 \mathrm{~h}$.

\subsubsection{Western Blotting}

To analyse the brain homogenate, the separated protein sample were transferred onto a nitrocellulose-membrane, where they are accessible for immunological detection of single protein species. 


\subsubsection{Electrophoretical Transfer}

The gel was placed in a "sandwich" of blotting paper (Whatman) and a nitrocellulose membrane (Whatman $\mathrm{GmbH}$ ) in a way that negatively charged protein samples are transferred in an electrical field towards the anode. The transfer was carried out in a submerged chamber (Hoefer Scientific Instruments) in transfer buffer (192mM glycine, $25 \mathrm{mM}$ Tris, $20 \%$ Methanol) at $4^{\circ} \mathrm{C}, 40 \mathrm{~mA}$, overnight.

\subsubsection{Ponceau S - Staining}

The success of the transfer was monitored by a reversible Ponceau-S-stain. The membrane was incubated in Ponceau-S-staining solution $(0.1 \%$ (w/v) Ponceau$\mathrm{S}, 5 \%$ Acetic Acid in $\mathrm{H}_{2} \mathrm{O}$ ), until protein bands could be observed and washed with water to remove excessive dye.

\subsubsection{Buffers and Solutions for Immunological Detection}

10x TBS:

1.37M NaCl, 200mM Tris-Base, $\mathrm{pH} 7.6$ with $\mathrm{HCl}$

Blocking buffer:

$5 \%$ milk powder, $5 \%$ goat serum (add only in blocking step), $0.1 \%$ Tween-20, 1x TBS

Washing buffer 1 :

1x TBS, 0.1\% Tween-20

Washing buffer 2:

1x TBS

\subsubsection{Immunological Detection}

\begin{tabular}{|l|l|l|}
\hline Step & Buffer & Time \\
\hline 1. Blocking & Blocking buffer + goat serum & 30min \\
\hline 2. Primary antibody & $\begin{array}{l}\text { Primary antibody dilution in } \\
\text { blocking buffer }\end{array}$ & $\begin{array}{l}\text { 1h, membrane sealed in } \\
\text { plastic bag }\end{array}$ \\
\hline 3. Washing & Blocking buffer & Two brief rinses \\
\hline
\end{tabular}




\begin{tabular}{|l|l|l|}
\hline & Blocking buffer & $15 \mathrm{~min}$ \\
\hline & Blocking buffer & $2 \times 5 \mathrm{~min}$ \\
\hline 4. Secondary antibody & $\begin{array}{l}\text { Secondary antibody dilution in } \\
\text { blocking buffer }\end{array}$ & $1 \mathrm{~h}$ \\
\hline & Blocking buffer & Two brief rinses \\
\hline & Blocking buffer & $1 \times 15 \mathrm{~min}$ \\
\hline & Blocking buffer & $2 \times 5 \mathrm{~min}$ \\
\hline & Wash buffer 1 & $1 \times 5 \mathrm{~min}$ \\
\hline & Wash buffer 2 & $1 \times 5 \mathrm{~min}$ \\
\hline
\end{tabular}

Table 2.6: Overview of the Procedure for the Immunological Detection

All steps were carried out at RT under mild agitation.

The membrane was placed on a glass plate and the ECL detection was performed according to the manufacturer's instructions. After reaction of the substrate, a plastic foil was laid on the membrane and Amersham Hyperfilms were exposed at desired times and developed with a developer (Agfa Curix 60).

\subsection{Electrophysiological Analysis}

\subsubsection{Solutions and Media}

$5 x$ Stock Slicing Solution:

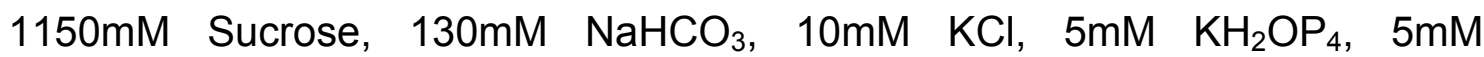
$\mathrm{MgCl}_{2} \times 6 \mathrm{H}_{2} \mathrm{O}, 50 \mathrm{mM}$ Glucose $\times \mathrm{H}_{2} \mathrm{O}$. Solution was sterilized by filtration through a $0.22 \mu \mathrm{m}$ filter and stored at $4{ }^{\circ} \mathrm{C} . \mathrm{CaCl}_{2}$ as added to $0.5 \mathrm{mM}$ final concentration to $1 \mathrm{x}$ slice solution from a $1 \mathrm{M}$ stock.

10x Stock Recording and Slice Storing Solution (ACSF):

$1200 \mathrm{mM} \mathrm{NaCl}, 260 \mathrm{mM} \mathrm{NaHCO} 3,10 \mathrm{mM} \mathrm{KH}_{2} \mathrm{PO}_{4}, 20 \mathrm{mM} \mathrm{KCl}, 20 \mathrm{mM}$ $\mathrm{MgCl}_{2} \times 6 \mathrm{H}_{2} \mathrm{O}, 100 \mathrm{mM}$ Glucose $\times \mathrm{H}_{2} \mathrm{O}$. Solution was sterilized by filtration through a $0,22 \mu \mathrm{m}$ filter and stored at $4{ }^{\circ} \mathrm{C}$. $\mathrm{CaCl}_{2}$ was added to $2 \mathrm{mM}$ final concentration to $1 \mathrm{x}$ solution from a $1 \mathrm{M}$ stock. 
1x Slicing Solution1.1:

$75 \mathrm{mM}$ Sucrose, $64 \mathrm{mM} \mathrm{NaCl}, 25 \mathrm{mM} \mathrm{NaHCO} 3,2.5 \mathrm{mM} \mathrm{KCl}, 1.25 \mathrm{mM}$ $\mathrm{NaH}_{2} \mathrm{OP}_{4} \times \mathrm{H}_{2} \mathrm{O} 7 \mathrm{mM} \mathrm{MgCl} 2 \times 6 \mathrm{H}_{2} \mathrm{O}, 25 \mathrm{mM}$ Glucose $\times \mathrm{H}_{2} \mathrm{O}, 0.5 \mathrm{mM} \mathrm{CaCl}$. Solution was sterilized by filtration and stored at $4^{\circ} \mathrm{C}$. $\mathrm{pH} 7.4,300 \mathrm{mOsm}$

1x External Solution 2.1:

$125 \mathrm{mM} \mathrm{NaCl}, 25 \mathrm{mM} \mathrm{NaHCO}_{3}, 2.5 \mathrm{mM} \mathrm{KCl}, 1.25 \mathrm{mM} \mathrm{NaH}_{2} \mathrm{OP}_{4} \mathrm{xH}_{2} \mathrm{O} 1 \mathrm{mM}$ $\mathrm{MgCl}_{2} \times 6 \mathrm{H}_{2} \mathrm{O}, 25 \mathrm{mM}$ Glucose $\times \mathrm{H}_{2} \mathrm{O}, 2 \mathrm{mM} \mathrm{CaCl}$. Solution was sterilized by filtration and stored at $4^{\circ} \mathrm{C}$. $\mathrm{pH} 7.4,300 \mathrm{mOsm}$

All extracellular solutions were equilibrated with carbogen gas $\left(95 \% \mathrm{O}_{2}, 5 \% \mathrm{CO}_{2}\right)$ before use and during use.

Biocytin Intracellular Solution:

138mM K-Gluconate, $16.8 \mathrm{mM} \mathrm{HEPES}$ free acid, $10 \mathrm{mM} \mathrm{KCl}, 1 \mathrm{mM} \mathrm{MgCl} 2 \times 6 \mathrm{H}_{2} \mathrm{O}$, $0.25 \mathrm{mM}$ EGTA pH $7.4,4 \mathrm{mM}$ ATP-Mg salt, $0.3 \mathrm{mM}$ Na-GTP, $0.2 \%$ Biocytin hydrochloride, $\mathrm{pH} 7.4$ with $\mathrm{KOH}, 300 \mathrm{mOsm}$

Standard Intracellular Solution:

136mM KCl, $17.8 \mathrm{mM}$ HEPES, 1mM EGTA, 1x ATP/GTP regeneration system $\left(10 \mathrm{x}=6 \mathrm{mM} \mathrm{MgCl}_{2}, 40 \mathrm{mM}\right.$ ATP-Mg ${ }^{2+}, 3 \mathrm{mM}$ GTP-Na $2,150 \mathrm{mM}$ Creatinephosphate, 50 $\mathrm{U} / \mathrm{ml}$ Creatinephosphokinase)

Intracellular solutions were sterile filtered through a $0.22 \mu \mathrm{m}$ filter and stored in syringes. Solutions were filled into the recording pipette through a $0.22 \mu \mathrm{m}$ filter again.

Drugs were used at the following concentrations and diluted in $1 \mathrm{x}$ extracellular recording solution:

\begin{tabular}{|c|c|}
\hline Drug & Final concentration \\
\hline Kainate $\left(10 \mathrm{mM}\right.$ in $\left.\mathrm{H}_{2} \mathrm{O}\right)$ & $\begin{array}{l}50,100 \text { or } 200 \mathrm{nM} \text { (interface) } \\
10 \mu \mathrm{M} \text { (submerged) }\end{array}$ \\
\hline $\mathrm{NBQX}\left(10 \mathrm{mM}\right.$ in $\left.\mathrm{H}_{2} \mathrm{O}\right)$ & $10 \mu \mathrm{M}$ \\
\hline TTX $\left(3 \mathrm{mM}\right.$ in $\left.\mathrm{H}_{2} \mathrm{O}\right)$ & $3 \mu \mathrm{M}$ \\
\hline
\end{tabular}

Table 2.7: Drugs Used in this Study 


\subsubsection{Preparation of Acute Slices}

Mice (P18 - P23) were anesthetized by Isoflurane (DeltaSelect) and decapitated. The brain was quickly dissected and put into ice-cold, sucrose based slicing solution that was bubbled with carbogen gas. Brains were trimmed and serial horizontal $(400 \mu \mathrm{m}$, gamma oscillations recording) or thalamocortical sections $(300 \mu \mathrm{m}$ for mIPSC recording and filling of $n R T$ cells with biocytin) (modified after Agmon and Connors, 1991) were cut using a vibratome (Leica VT1200S). During the sectioning the tissue was immersed in ice-cold sucrose based solution and oxygenated with carbogen. The sections were transferred to a homemade holding chamber and kept in submerged or interface (for gamma oscillations) conditions for $1 \mathrm{~h}$ at $30^{\circ} \mathrm{C}$ before recording. The solutions were continuously bubbled with carbogen gas.

For recording of gamma oscillations in submerged conditions, the mouse was briefly anesthetized with Isoflurane, followed by an ip injection of ketamine/xylazine $\left(120 \mathrm{mg}^{*} \mathrm{~kg}^{-1} / 8 \mathrm{mg}^{*} \mathrm{~kg}^{-1}\right)$. Animals were transcardially perfused with around $50 \mathrm{ml}$ icecold, sucrose-based slicing solution. The brain was removed and processed as above.

\subsubsection{Patch-clamp Recordings}

Slices were transferred into submerged recording chamber and weighed down with a U-shaped platinum wire across which nylon threads had been strung. Recordings were performed at RT or at $30^{\circ} \mathrm{C}$ for filling of neurons with biocytin. A low-magnification objective $(4 \mathrm{x})$ was used to identify the $\mathrm{nRT}$ region and single $\mathrm{nRT}$ cells were identified by infrared-differential interference contrast with the help of a high-magnification water immersion objective (60x) using an upright Olympus BX51WI microscope equipped with a Zeiss Axio cam MRm camera and the corresponding AxioVision Software. Recording pipettes were pulled from $1.5 \mathrm{~mm}$ outer diameter borosilicate glass capillaries using a Sutter 2000 filament based horizontal puller. When filled with internal solution (see above) they had a resistance between $2.5-5 \mathrm{M} \Omega$. Recordings were carried out using an HEKA double patch clamp EPC 10 amplifier with the corresponding HEKA Patchmaster software. Series resistance during recording was between 6 and $25 \mathrm{M} \Omega$. Signals were filtered with a $10 \mathrm{kHz}$ Bessel filter. 
Cells were identified by their location within the boundaries of the nucleus, the shape of the soma and the firing pattern, after applying depolarizing current steps via the recording pipette. GABAergic miniature IPSCs were recorded in $\mathrm{nRT}$ cells at a holding potential of $-70 \mathrm{mV}$ in the presence of TTX (to prevent the generation of action potentials) and NBQX (to block AMPA and kainate receptor induced postsynaptic currents). Analysis of mIPSCs was performed with MiniAnalysis software (Synaptosoft) and Kalaidograph.

Whole-cell patch-clamp recordings from $\mathrm{nRT}$ cells from thalamocortical sections were done at $30^{\circ} \mathrm{C}$, with Biocytin included in the internal solution. Again, cells were identified within the boundaries of the nucleus and the shape of the soma. The firing pattern of the cells was recorded by applying depolarizing current steps from a holding potential of $-70 \mathrm{mV}$ (starting at $-200 \mathrm{pA}$ with $100 \mathrm{pA}$ increments for $1 \mathrm{sec}$ ). Cells were kept in whole cell mode for $30 \mathrm{~min}$ in order to allow a thorough diffusion of Biocytin in the cell. The recording pipette was carefully withdrawn and the slice was kept in the recording chamber for additional $15 \mathrm{~min}$ in order to allow a complete filling of the neuron. Afterwards, the slice was fixed in 4\% PFA overnight (see 2.9)

\subsubsection{Extracellular Field Recordings}

\subsubsection{Gamma Oscillations in Submerged Conditions}

For recording of gamma oscillations, slices were kept in an interface of the storage solution and a carbogen-saturated environment in the holding chamber. In order to induce gamma oscillations, slices were elevated in the recording chamber, by placing the slice on a nylon mesh in the chamber. Additionally, the perfusion rate with extracellular solution was increased (to around $3-5 \mathrm{ml}^{*} \mathrm{~min}^{-1}$ ) and the bubbling rate with carbogen was enhanced. The recordings were performed at $30^{\circ} \mathrm{C}$. The recording electrode (see above) was filled with $1 \mathrm{M} \mathrm{NaCl}$ and placed in the pyramidal cell layer of the CA3b region of the hippocampus under visual control at a depth of about $100 \mu \mathrm{m}$. Field recordings were recorded for $5 \mathrm{~min}$ with a HEKA double patch clamp EPC 10 amplifier and filtered at $10 \mathrm{kHz}$. Oscillations in the gamma frequency range were generated by application of $10 \mu \mathrm{M}$ Kainate in extracellular solution via a homemade T-valve that was placed in close proximity to the recording electrode. 
Recorded data were analysed with Axograph X (Axon Instruments). The traces were filtered offline at $100 \mathrm{kHz}$ and power spectra were calculated by Fourier Transformation for the period of oscillatory activity occurring in the field potential. The power spectrum resulting from baseline (without Kainate) recording was subtracted from the trace with Kainate application. The subtracted trace was used to determine the frequency of the maximum peak, the maximum peak power and the integral area power in the frequency band from 25 to $45 \mathrm{~Hz}$.

\subsubsection{Gamma Oscillations in Interface Conditions}

After slicing, the sections were kept floating on the surface of the extracellular recording solution in a homemade holding chamber, saturated with carbogen gas. Network activity was recorded in $400 \mu \mathrm{m}$ thick hippocampal slices cut in horizontal orientation in an interface recording chamber (BSC-BU Base Unit with the BSC-HT Haas Top, Harvard apparatus) at $33^{\circ} \mathrm{C}$. Slices were kept on a nylon mesh at the interface of a stream of extracellular recording solution and a stream of warm, moist carbogen gas. Extracellular recording electrodes with a resistance of around $2 \mathrm{M} \Omega$ were filled with extracellular solution and placed in the pyramidal cell layer of the $\mathrm{CA} 3 \mathrm{~b}$ region in the hippocampus. Extracellular field potentials were recorded with a MultiClamp 700B amplifier (Axon, Molecular Devices) and the Digidata $1440 \mathrm{~A}$ (Axon, Molecular Devices), filtered at $3 \mathrm{kHz}$ with a Bessel filter and a gain of 100 . Gamma oscillations were induced by bath application of 50, 100 and 200nM Kainate sequentially on the same slice. Stable and long lasting oscillatory activity could be recorded 5-7 min after the start of Kainate application. Baseline field-potentials and gamma-oscillation field potentials for each concentration of Kainate were recorded for 16min. Data were analysed with Axograph X (Axon Instruments). Traces were filtered at $100 \mathrm{kHz}$ and power spectra were calculated for eight-minute epochs (last 8 minutes of each recording) of recorded field activity. Baseline power spectra were subtracted from Kainate traces and the frequency and the power of the maximum peak was determined. The power of the frequency band $25-45 \mathrm{~Hz}$ was calculated as the area under the respective peak in the power spectrum. 


\section{Results}

\subsection{Viaat Conditional Knockout Mice}

The Viaat conditional knockout allele $\left(\right.$ Viaat $\left.^{\mathrm{fl} / \mathrm{fl}}\right)$ was generated by Dr. S. Wojcik. The targeting vector for the genomic recombination was generated by a recombineering approach in E.coli (Liu et al., 2003). The Viaat ${ }^{\mathrm{fl} / \mathrm{fl}}$ animals are viable, develop normally and are indistinguishable from wild type (wt) littermates. To determine whether the presence of the loxP sites influences the expression of the Viaat gene, total brain Viaat protein levels of heterozygous $(\mathrm{f} / /+)$ and homozygous (fl/fl) animals carrying the loxP-flanked (floxed) allele were compared to the protein levels of wt littermates by Western blotting. Whole brain homogenate, transferred to a nitrocellulose membrane was probed with an antibody against Viaat. An antibody to the synaptic vescicle protein synaptophysin was used as an internal loading control. The presence of the loxP sites, which flank the second exon of the Viaat gene, did not have any apparent effect the expression of the gene, as Viaat protein levels of Viaat $^{\mathrm{fl} / \mathrm{+}}$ and Viaat ${ }^{\mathrm{f} / \mathrm{fl}}$ animals were indistinguishable from those of wt animals (Figure $3.1 \mathrm{~B})$.

A

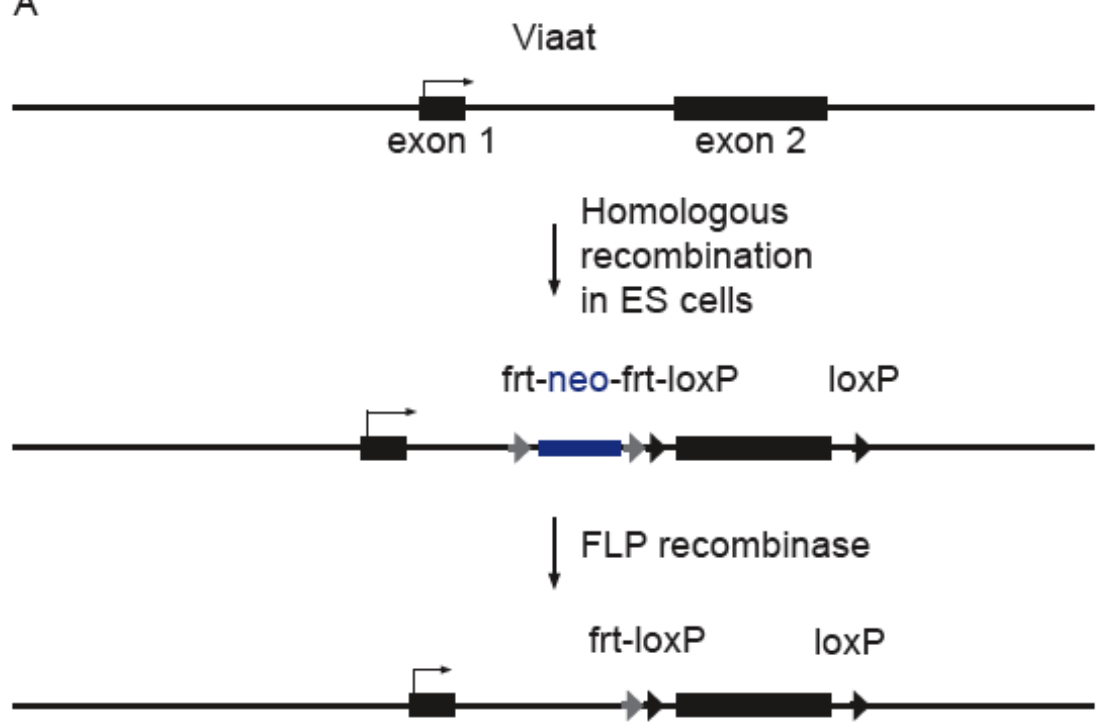

B

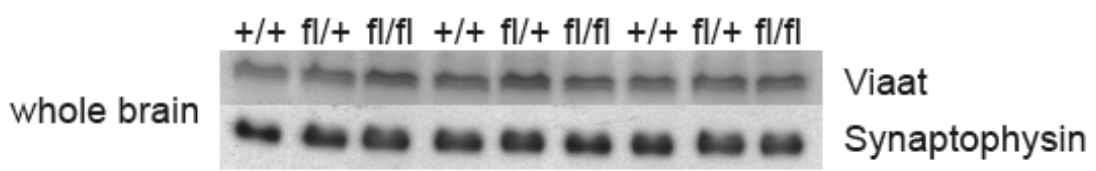

Figure 3.1: Viaat Conditional Knockout Allele 
(A) Summarized strategy for the generation of the conditional floxed Viaat allele by homologous recombination in ES-cells. The neomycin cassette used for selection of recombinant clones in ES cells was flanked by recognition sites for the FLPrecombinase (frt sites) and removed by breeding the first generation of animals to animals expressing FLP-recombinase (B) The presence of the loxP sites did not influence the expression of the Viaat gene as analysed by Western Blotting of whole brain homogenate from wt $(+/+)$, heterozygous $(\mathrm{fl} /+)$ and homozygously $(\mathrm{fl} / \mathrm{fl})$ floxed animals.

The conditional Viaat knockout mouse line was designed with the aim of crossing it to transgenic mouse lines expressing Cre in distinct subset of GABAergic neurons. This strategy makes it possible to delete the Viaat gene in a spatially and temporally controlled manner, depending which Cre-expression pattern is combined with the conditional knockout allele (Nagy, 2000).

\subsection{Shutdown of GABA Release in Cultured Viaat ${ }^{\mathrm{fl} / \mathrm{fl}}$ Neurons upon Expression of Cre-recombinase}

Genetic deletion of vesicular neurotransmitter transporters leads to a shutdown of neurotransmitter release, i.e. to a silencing of the neurons since only empty vesicles are released. This has been demonstrated for the vesicular transporters for glutamate, VGLUT1 (Fremeau et al., 2004; Wojcik et al., 2004), VGLUT2 (Moechars et al., 2006; Wallen-Mackenzie et al., 2006) and GABA/glycine (Mclntire et al., 1993; Wojcik et al., 2006). To confirm that expression of Cre-recombinase in neurons with the Viaat ${ }^{f l / f l}$ allele would result in a shutdown of GABA release, striatal neurons, which are mainly GABAergic, from Viaat ${ }^{\mathrm{fl} / \mathrm{fl}} \mathrm{PO}$ animals were cultured as single-neuron autaptic cultures (Pyott and Rosenmund, 2002) and as dense, confluent cultures. These cultures were infected with a lentivirus expressing either Cre-recombinase and EGFP or EGFP alone as control (Figure $3.2 \mathrm{~A}$ ). After 12 days in-vitro, immunofluorescence analyis revealed that the typical punctate Viaat signal (red) seen in control cultures is absent in the Viaat ${ }^{f / / f l}$ cells expressing Cre-recombinase (Figure $3.2 \mathrm{~B}$ ). Confluent cultures were lysed and used for biochemical analysis of Viaat expression. No Viaat signal could be detected by Western Blot analysis in Creinfected neurons, while it was readily detected in EGFP-infected and in non-infected neurons. Antibodies to Gephyrin and Rim1 $\alpha$ were used as controls, and showed no 
difference between cultures expressing Cre and EGFP, EGFP alone or uninfected neurons (Figure $3.2 \mathrm{C}$ ).

Dr. Rhee performed the electrophysiological analysis of the infected neurons. 12 days after infection, GABA release could be still detected, although it was reduced in the Cre-infected neurons (data not shown). After 20 days most of the neurons showed no evoked or very small IPSC responses (Figure 3.2 D), essentially producing a phenocopy of the conventional Viaat knockout. Thus, the Viaat ${ }^{\mathrm{fl} / \mathrm{fl}}$ allele functions as expected and GABA release is eliminated in neurons that express Crerecombinase.

A
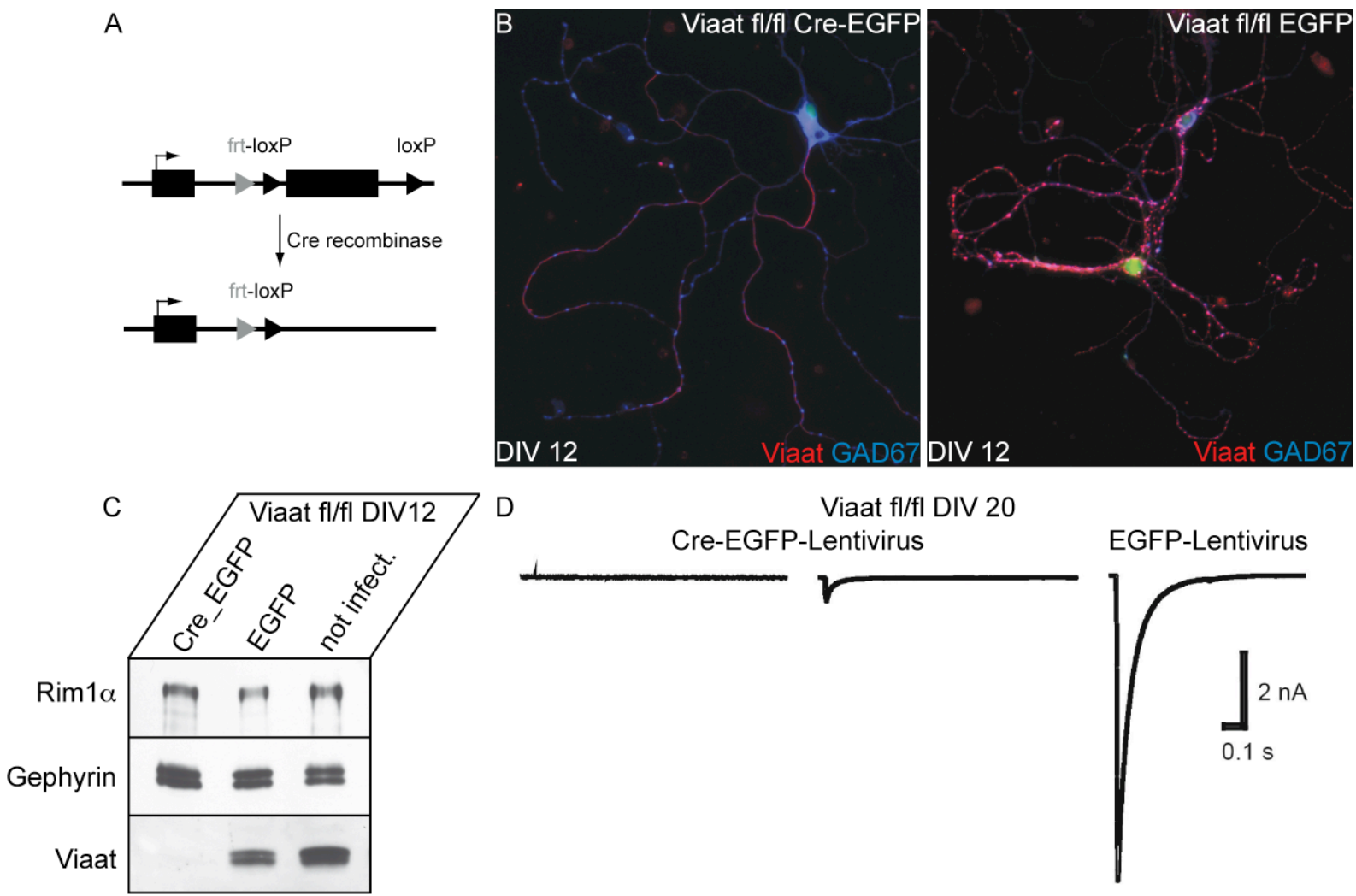

Figure 3.2: GABA Release is Eliminated in Cre-infected Viaat $\mathrm{f}^{\mathrm{fl} / \mathrm{fl}}$ Neurons

(A) Strategy used to delete Viaat in cultured neurons. Cells were infected with lentivirus expressing Cre-recombinase in order to delete Viaat. (B) Immunostaining of neurons with antibodies directed against Viaat (red) revealed the loss of the protein in Cre-infected neurons, whereas punctate Viaat immunoreactivity was apparent in lentivirus-EGFP infected control cultures. GAD67 (blue) was used as a control and marker for inhibitory synapses. (C) Western Blot analysis showed the loss of Viaat in Cre-infected neuron cultures. Rim $1 \alpha$ and Gephyrin were used as controls and did not show any changes in protein levels. (D) Raw traces of evoked IPSCs from Creinfected and EGFP infected neurons show that GABA release is shut down or drastically reduced in Viaat ${ }^{\mathrm{fl} / \mathrm{fl}}$ neurons expressing Cre. 


\subsection{Conditional Viaat Knockout in Parvalbumin Expressing}

\section{Neurons}

To study the effects of deleting Viaat from a major class of fast-spiking GABAergic interneurons, the Viaat ${ }^{f / f 1}$ mouse line was crossed with a mouse line expressing a conventional Cre-recombinase under the control of the Parvalbumin (PV) promoter. With that approach it is possible to delete Viaat only in PV-expressing neurons, thereby affecting their ability to release GABA. Only those cell types that express both the PV-Cre transgene and Viaat are affected by the gene deletion, whereas all other cells are unaffected (Figure 3.3 A). The PV-Cre transgenic mouse line was generously provided by Prof. Monyer (Fuchs et al., 2007; Monyer and Markram, 2004).

The Viaat ${ }^{f / / 1}, P V-C r e$ animals are born and develop normally in the first two weeks of life. By three weeks of age, the mice develop an obvious motor phenotype and hind limb clasping (Figure 3.3 B). They move less and appear uncoordinated in the home cage compared to control littermates. At the end of the three weeks Viaat ${ }^{f / f l}, \mathrm{PV}$-Cre animals are prone to epileptic seizures with loss of motor control and complete body stiffness in response to handling. The animals frequently do not survive these events and the majority of animals die around the age of three weeks. The observed lifespan ranged from 18 days to four weeks of age. Animals that survive past three weeks appear to move around less in the cage and also tend to have a lower body weight. Because of the dramatic phenotype, animals were selected for analysis around P19. 
A

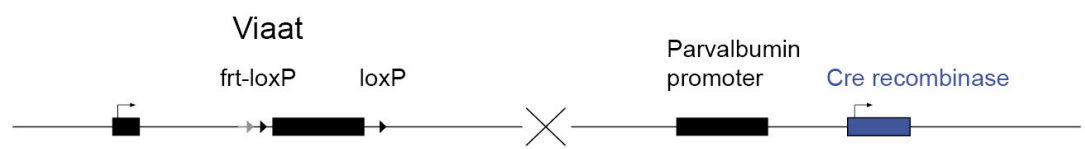

In parvalbumin expressing cells

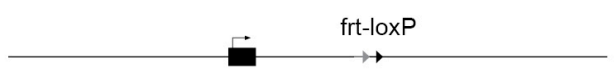

In parvalbumin non-expressing cells

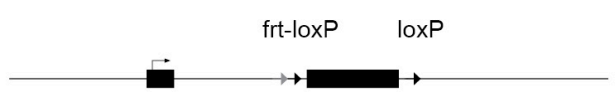

B

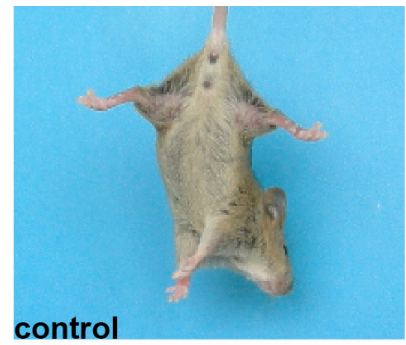

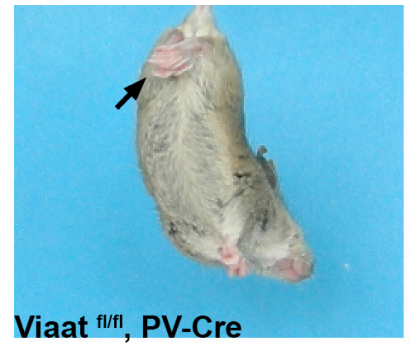

Figure 3.3: Targeting of Parvalbumin Expressing GABAergic Neurons

(A) The combination of the conditional Viaat allele with a mouse line expressing Crerecombinase under the control of the PV-promoter allows the selective deletion of Viaat in PV-expressing GABAergic neurons. (B) Hind limb clasping was reliably observed in Viaat ${ }^{\mathrm{fl} / \mathrm{fl}}$, PV-Cre animals.

\subsection{Expression Pattern of the PV-Cre Transgene}

To characterize the expression pattern of the PV-Cre transgene in the Viaat ${ }^{\mathrm{fl} / \mathrm{fl}}$, PV-Cre animals, they were crossed with mice carrying a Cre-reporter transgene consisting of a cytomegalovirus-beta-actin-promoter (CAG) followed by a loxP flanked chloramphenicol transferase stop cassette (CAT) and EGFP. Throughout this text, the acronym CGCT is used for the mouse line carrying this CAG-CAT-EGFP Cre-reporter transgene. In this mouse line, Cre-expressing cells express EGFP after Cre-mediated excision of the floxed CAT stop cassette (see Figure 3.4 A) (Nakamura et al., 2006). Since excision of the CAT stop cassette and the loxP flanked target gene, in this case the Viaat gene, is assumed to occur in the same cells and roughly at the same time, expression of EGFP can thus be used as a marker for cells in which the target gene has been deleted. 
To assess the expression pattern of the PV-Cre transgene, double-labelling immunofluorescence experiments were carried out to analyse the co-expression of PV and EGFP (indicating Cre-mediated recombination due to the expression of the PV-Cre) in different brain regions and neurons as depicted in Figure 3.4 B - G. 
A

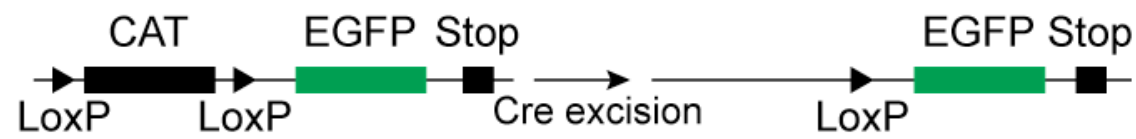

B

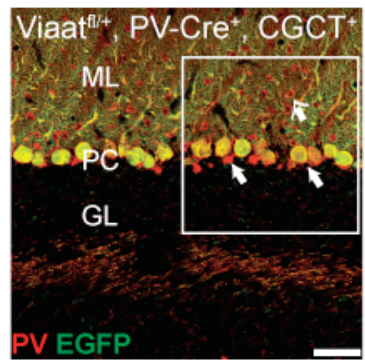

C

Viaat ${ }^{1+4}$, PV-Cre', CGCT

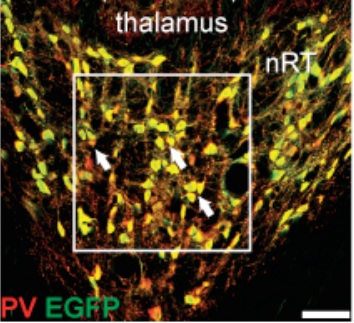

D Viaat ${ }^{1 /+}, \mathrm{PV}^{-\mathrm{Cre}^{+}}, \mathrm{CGCT}^{+}$

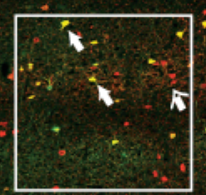

DVEGFP

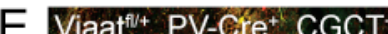

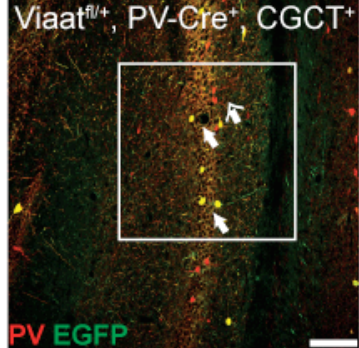

F

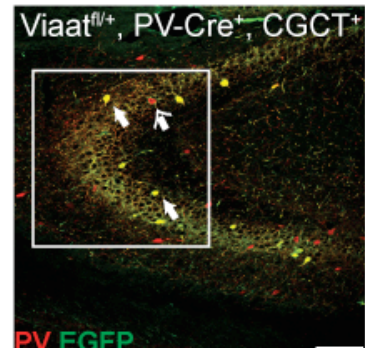

G Viaat ${ }^{\mathrm{fl}+}, \mathrm{PV}^{-} \mathrm{Cre}^{+}, \mathrm{CGCT}^{+}$

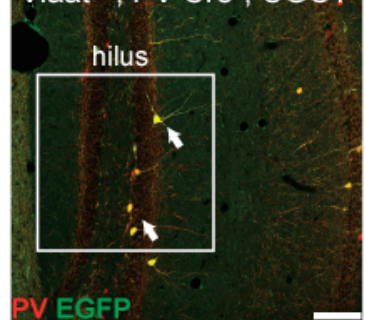

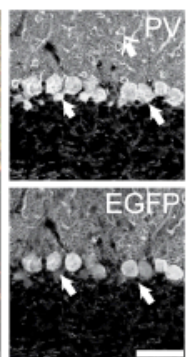
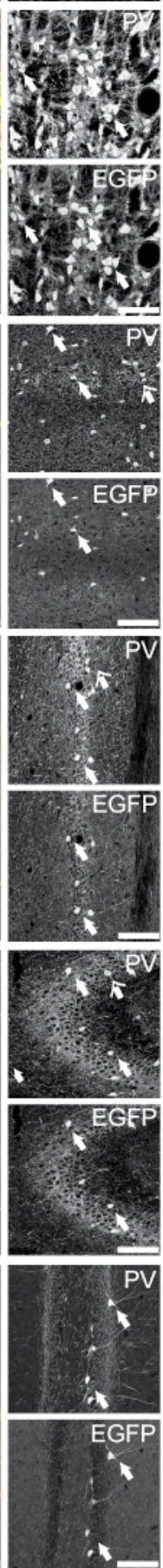

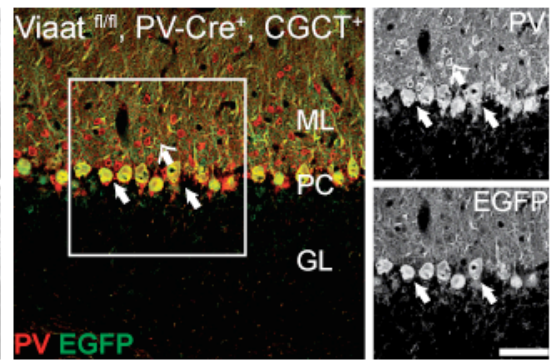

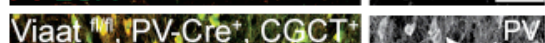
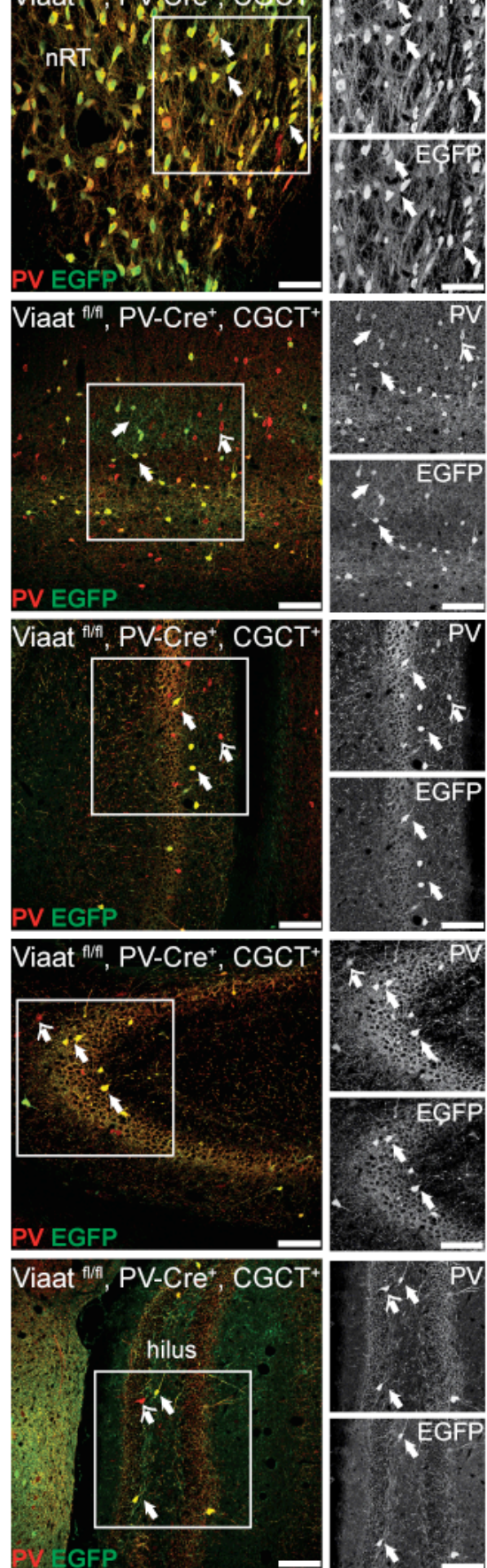
Figure 3.4: Expression of the PV-Cre Transgene in Different Brain Regions (A) Mice carrying the CGCT-transgene express EGFP after Cre-mediate excision of the CAT-stop-cassette. (B-G) Coronal brain sections of control (Viaat ${ }^{\mathrm{fl} /+}$, PV-Cre,

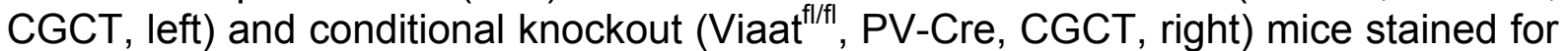
PV (red) and EGFP (green). Grey-scale images: details of PV- (upper panel) and EGFP-labelling (lower panel) alone. (B) Cerebellum. (C) Reticular Thalamic Nucleus. (D) Cortex. (E) Hippocampus CA1. (F) Hippocampus CA3. (G) Hippocampus DG. $\mathrm{ML}$, molecular layer; PC, Purkinje cell layer; GL, granule cell layer; $n R T$, reticular thalamic nucleus; closed arrowheads: examples of co-localisation of PV and EGFP. Open arrowheads: only PV immunoreactivity. Scale bar: B, C: $50 \mu \mathrm{m}$; D-G: $100 \mu \mathrm{m}$.

The degree of co-localization of endogenous PV and EGFP as a marker of PVCre transgene expression differed between brain regions and cell types. All cerebellar Purkinje cells, which are GABAergic projecting neurons, show a prominent co-labelling of PV and EGFP, indicating that Cre-mediated recombination has taken place in the entire population of Purkinje cells. However, no EGFP positive GABAergic interneurons of the molecular layer (Basket cells and Stellate cells) were observed (Figure $3.4 \mathrm{~B}$ ). The second region with a high rate of co-localisation of PV and EGFP is the reticular thalamic nucleus (nRT). Almost $100 \%$ of the cells in the $\mathrm{nRT}$, which are also GABAergic projecting neurons, show EGFP immunoreactivity indicative of Cre-activity (Figure $3.4 \mathrm{C}$ ).

By comparison, the expression of the PV-Cre transgene in PV-positive GABAergic interneurons of the cortex matches the expression pattern of endogenous PV less well. In the cortex, only a minority of PV-positive cells shows EGFP immunoreactivity (Figure $3.4 \mathrm{D}$ ). In the hippocampal region however, a higher proportion of interneurons show co-expression of PV-Cre and endogenous PV. EGFP positive neurons could be found in all subregions of the hippocampus including the CA1 and CA3 subfield as well as the dentate gyrus (Figure 3.4 E, F, G). PV expressing cells in the hippocampus can be found in the pyramidal cell layer, stratum oriens but also the stratum radiatum and stratum lancosum-moleculare in the CA1 and CA3 fields. EGFP positive cells were observed throughout these regions. The majority of the EGFP expressing cells were found in the stratum pyramidale and the stratum oriens, to a lower extend in the stratum radiatum. EGFP - PV double positive cells of the dentate gyrus could be observed in the stratum granulosum and at the border between the hilus region and the granular cell layer.

Throughout the brain, expression of the PV-Cre transgene was restricted to PVexpressing neurons, but, with the exception of Purkinje cells and the cells of $n R T$, 
expression of the transgene did not extend to all neurons expressing endogenous PV in a given region. Some brain regions, such as the olfactory bulb, expressed endogenous PV, but did not show any EGFP immunostaining (data not shown).

Overall, this analysis showed that expression of the PV-Cre transgene matches the distribution of endogenous PV (Celio, 1990) and confirmed the high degree of coexpression of PV-Cre and endogenous $\mathrm{PV}$ in the hippocampus reported by the lab that generated the PV-Cre transgenic mice (Fuchs et al., 2007).

No differences in the distribution of EGFP positive cells were observed between heterozygous control animals (Viaat ${ }^{\mathrm{fl} /+}, \mathrm{PV}-\mathrm{Cre}+, \mathrm{CGCT}+$ ) and conditional knockoutmice (Viaat ${ }^{\mathrm{fl} / \mathrm{fl}}, \mathrm{PV}-\mathrm{Cre}+, \mathrm{CGCT+}$ ). The expression level of EGFP is high enough to allow visualization of EGFP-positive cells without staining in the acute slices used for electrophysiological experiments.

\subsection{Loss of Viaat in Viaat ${ }^{\mathrm{fl} / \mathrm{fl}}$, PV-Cre Neurons}

To selectively delete Viaat in PV-positive neurons, mice carrying the floxed Viaat allele $\left(\right.$ Viaat $\left.{ }^{f / f l}\right)$ were crossed with transgenic animals expressing Crerecombinase under the control of the PV-promoter (PV-Cre). The analysis of the selective ablation of Viaat from the target neurons was done by double immunofluorescence analysis. The co-localisation of Viaat with synaptic and postsynaptic markers (GAD67, GAD65, Gephyrin) was analysed in the cerebellum, thalamus and the hippocampus. Almost $100 \%$ of the cerebellar Purkinje cells were affected by the cell-type specific deletion of Viaat (see Figure $3.4 \mathrm{~B}$ ). The primary synaptic target regions of these cells are neurons of the deep cerebellar nuclei. These cells receive massive GABAergic input from the cerebellar Purkinje cells (Teune et al., 1998). The immunohistological analysis revealed that Viaat immunoreactivity surrounding the cells of the deep cerebellar nuclei in control animal was absent in Viaat ${ }^{\mathrm{flfl}}$, PV-Cre animals (see Figure 3.5 A). In order to analyse if the number of synapses was affected by the loss of Viaat, Gephyrin positive puncta surrounding the cell soma as well as Gephyrin positive puncta in the whole image were counted. Quantification of whole images did not reveal any differences (11.5 \pm 0.3 puncta per $100 \mu \mathrm{m}^{2}$ in Viaat ${ }^{\mathrm{f} / \mathrm{fl}}$, PV-Cre compared to $12.0 \pm 0.3$ puncta per $100 \mu \mathrm{m}^{2}$ in controls.) However, a small but significant difference of Gephyrin immunoreactivity was detected when only the perisomatic puncta were counted. The average puncta 
number in brain sections from Viaat ${ }^{\mathrm{fl} / \mathrm{fl}}, \mathrm{PV}$-Cre animals was $2.6 \pm 0.1$ per $10 \mu \mathrm{m}^{2}$ ( $n=79$ cells, 3 animals) compared to $2.9 \pm 0.1$ puncta per $10 \mu m^{2}$ in controls $(n=72$ cells, 3 animals) $p=0.02$ (Figure $3.5 \mathrm{~A}$ and $\mathrm{A} 1$ )

A second region analysed for the loss of Viaat immunoreactivity was the target area of the neurons of the $\mathrm{nRT}$. Cells from the $\mathrm{nRT}$ project to almost all thalamic nuclei. The target region depends on the localization of the $\mathrm{nRT}$ cell within the nucleus (Pinault and Deschenes, 1998). A complete loss of Viaat protein in the thalamus could be observed in the ventral basal complex, consisting of the ventral posteromedial thalamic nucleus (VPM) and ventral posterolateral thalamic nucleus $(\mathrm{VPL})$ in the Viaat ${ }^{\mathrm{fl} / \mathrm{fl}}, \mathrm{PV}-\mathrm{Cre}$ animals (see Figure $3.5 \mathrm{~B}$ ). In order to ensure the analysis of the correct synapses, a co-labelling of Viaat and EGFP (indicates Creactivity due to the presence of the reporter allele) was carried out in control animals and Viaat ${ }^{f / f l}$, PV-Cre animals. As depicted in Figure $3.5 \mathrm{C}$, in control animals, colabelling of both proteins (Viaat in red, EGFP in green) was observed, which proves the presence of synapses from neurons of the nRT. The loss of Viaat in Viaat ${ }^{f / f l}, P V-$ Cre mice was confirmed in this analysis, as well.

The immunofluorescence analysis shows that the conditional knockout allele functions as expected and that Viaat expression was eliminated in the brain regions with the most prominent expression of PV-Cre. 



Figure 3.5: Loss of Viaat Immunoreactivity in Deep Cerebellar Nuclei and in the Thalamus

(A) Selective loss of perisomatic Viaat labeling in the deep cerebellar nuclei, the target region of cerebellar Purkinje cells as shown by co-labelling of Viaat (red) and Gephyrin (green) in Viaat ${ }^{\mathrm{f} / \mathrm{fl}}, \mathrm{PV}-\mathrm{Cre}$ vs. control animals. Grey scale images show Viaat (left) and Gephyrin (right) alone. Scale $=10 \mu \mathrm{m}$ (A1) Quantification of Gephyrin puncta in the whole image (upper) and perisomatic Gephyrin puncta around the cell soma (lower panel), shows a small reduction of perisomatic Gephyrin puncta. $\mathrm{n}=$ cell number from 3 animals each. (B) Loss of Viaat in the ventral basal complex of the thalamus, one target region of neurons of the $\mathrm{nRT}$ as analysed by co-labelling of Viaat (red) and GAD67 (green). Grey Scale images show Viaat (left) and GAD67 (right) alone, respectively. Scale $=20 \mu \mathrm{m}$, higher magnification $=10 \mu \mathrm{m}$ (C) Colabelling of Viaat and EGFP (indicates Cre-activity) in the VB-complex. Scale $=20 \mu \mathrm{m}$ 
The same analysis was carried out in the hippocampus to assess the expression of Viaat in PV-positive interneurons. However, no obvious loss of Viaatimmunoreactivity was observed in the CA1 region of the hippocampus (Figure 3.6). This may be due to the fact that in the hippocampus it is not possible to isolate a target region that receives input solely from PV-expressing GABAergic neurons with no input from other classes of inhibitory neurons that are not affected by the conditional gene deletion. Furthermore, the expression of PV-Cre in interneurons may start later in development than the expression in the cells of $\mathrm{nRT}$ and Purkinje cells (Celio, 1990) thus leaving less time for a dramatic reduction in Viaat protein levels that can easily be detected.
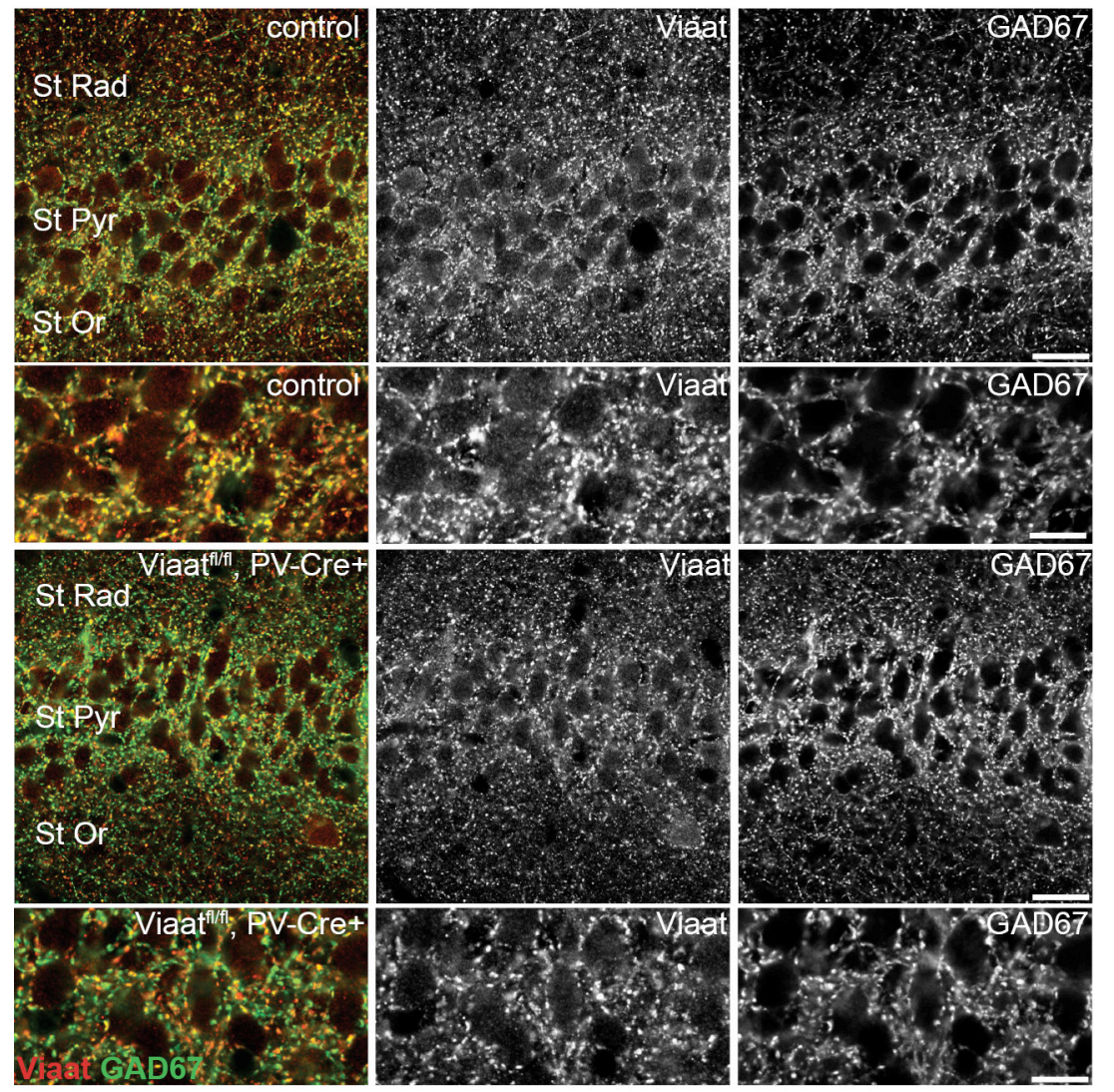

Hippocampus
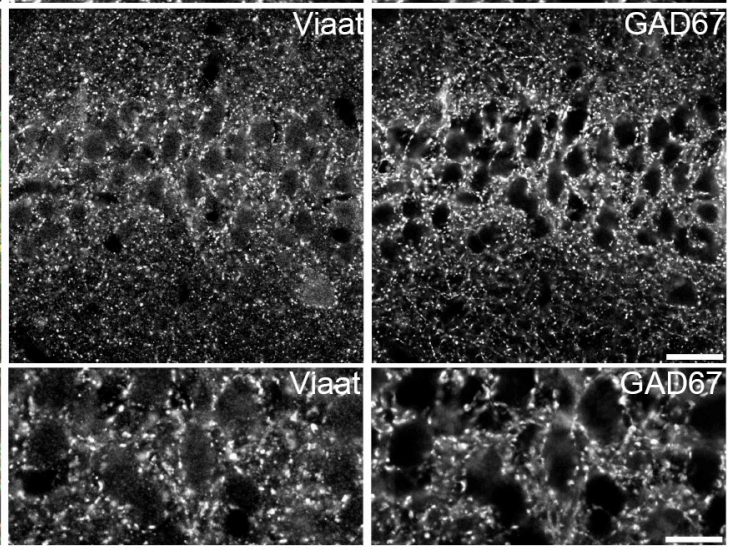

CA1

Figure 3.6: No Obvious Loss of Viaat Immunoreactivity in the Hippocampus

No obvious loss of Viaat immunoreactivity was observed in the hippocampus CA1 region by co-labelling of Viaat (red) and GAD67 (green). Grey scale images show Viaat (left) and Gad67 (right) alone. The higher magnification image shows the stratum pyramidale. St Rad, stratum radiatum; St Pyr, stratum pyramidale; St Or, stratum oriens. Scale $=20 \mu \mathrm{m}$, magnification $=10 \mu \mathrm{m}$. 


\subsection{Morphological Consequences of Deleting Viaat in Parvalbumin Positive Neurons}

GABAergic neurotransmission is thought to play a critical role in the development of neuronal networks. Furthermore, GABA is thought have a function as a trophic factor that acts before inhibitory neurotransmission, via chemical synapses, is established (reviewed in Represa and Ben-Ari, 2005; Sernagor et al., 2010). However, other studies where genes that are essential for GABAergic neurotransmissions were deleted globally, for example Viaat (Wojcik et al., 2006) or the GABA synthesizing enzymes GAD65 and GAD67 (Ji et al., 1999), contradict these findings. In these studies no change in the overall brain morphology was found.

To determine whether the loss of Viaat would result in a loss of other synaptic proteins, additional immunofluorescence analyses of synaptic marker proteins were performed. In the deep cerebellar nuclei, the region specifically targeted by Purkinje cells, Viaat ${ }^{\mathrm{fl} / \mathrm{fl}}$, PV-Cre animals showed no dramatic alterations in the staining intensity or staining pattern of other synaptic proteins in the absence of Viaat. Doubleimmunofluorescent staining of Synapsin (red, as a general synaptic marker) and the two GABA synthesizing enzymes GAD65 and GAD67 (in green) showed no obvious difference between the Viaat ${ }^{f / / f l}$, PV-Cre and control animals (Figure 3.7 A and B). Additional immunostaining of Viaat (red) and either PV (Figure $3.7 \mathrm{C}$ ) or EGFP (Figure $3.7 \mathrm{D}$ ), confirmed that the correct target region of cerebellar Purkinje cells and the correct synapses were analysed. A quantitative analysis of the GAD67 puncta in the deep cerebellar nuclei showed a small, but significant reduction of GAD67 immunoreactivity in the deep cerebellar nuclei $\left(8.1 \pm 0.3\right.$ puncta per $100 \mu \mathrm{m}^{2}$ in Viaat ${ }^{f / f f l}, P V-C r e$ animals, compared to $9.3 \pm 0.3$ puncta per $100 \mu m^{2}$ in control animals, $p=0.009, n=65$ control images, 53 Viaat $^{f l / f l}$, PV-Cre images from 3 animals each genotype).

Although the analysis of synaptic marker proteins in the absence of Viaat showed a small reduction in the number of GAD67-positive puncta, no dramatic effects of the absence of Viaat and consequently of the shutdown of GABA release on neuronal development and synapse formation of cerbellar Purkinje cells on their target cells in the deep cerebellar nuclei was observed. 


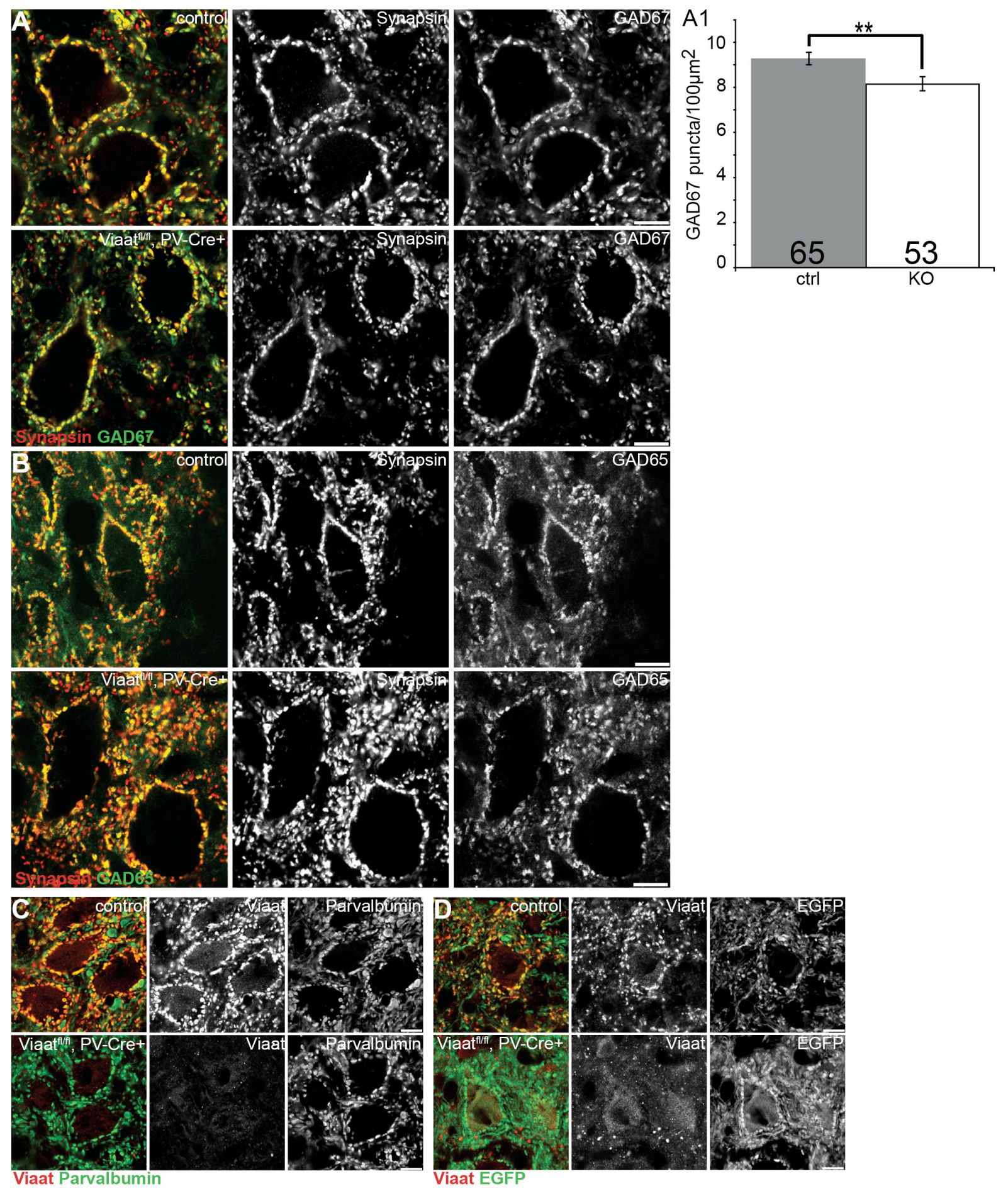

Figure 3.7: No Obvious Morphological Consequences of the Loss of Viaat in the Deep Cerebellar Nuclei

(A) No obvious change in the expression of synaptic marker proteins in the deep cerebellar nuclei as revealed by co-labelling of Synapsin (red) and GAD67 (green). Grey Scale images: Synapsin (right) and GAD67 (left) alone. (A1) Small, but significant reduction of GAD67 immunoreactivity in the deep cerebellar nuclei. (B) No obvious change in the expression of synaptic markers in the deep cerebellar nuclei as revealed by co-labelling of Synapsin (red) and GAD65 (green). Grey Scale images: Synapsin (right) and GAD65 (left) alone. (C) Co-localisation of Viaat (red) and PV (green) confirmed that the synapses analyzed originate from the Purkinje cells. Grey scale images show again the respective stainings alone. (D) Co-labelling 
of Viaat (red) and EGFP (green, indicated Cre-activity), confirms that the synapses analyzed are Viaat ${ }^{\mathrm{fl} / \mathrm{fl}}, \mathrm{PV}-\mathrm{Cre}$ synapses. Scale bars $=10 \mu \mathrm{m}$

\subsection{No Obvious Morphological Changes in Neurons of the Reticular Thalamic Nucleus}

To determine the morphological consequences of the genetic deletion of Viaat in more detail, cells from the reticular thalamic nucleus were filled with biocytin via whole cell patch clamp recordings in thalamocortical slices. Biocytin is distributed throughout the whole cell including the processes, which allows a more detailed visualization of the whole neuron. Two types of neurons could be classified in this preparation, based on the projection pattern of the axon into the thalamus. For one type, the axon projected into the VB region of the thalamus including the VPM and the VPL (Figure 3.8 A, B). The other type sent its axons deep into the thalamus into the POM region (Figure 3.8 C, D). Unfortunately, the staining of the axonal processes including the final synaptic field was not optimal, which precluded a detailed analysis. For both types of nRT neurons, two distinct forms of axonal arborisations could be observed. The axons formed either a relatively defined, compact synaptic field (Figure $3.8 \mathrm{~A}, \mathrm{~B}$ ) or showed a widespread and more diffuse axonic arborisation (Figure 3.8 C, D) (compare with Cox et al., 1996). However, no obvious differences in the axonal morphology could be observed between the Viaat ${ }^{\mathrm{fl} / \mathrm{fl}}, \mathrm{PV}-\mathrm{Cre}$ animals and control littermates. 


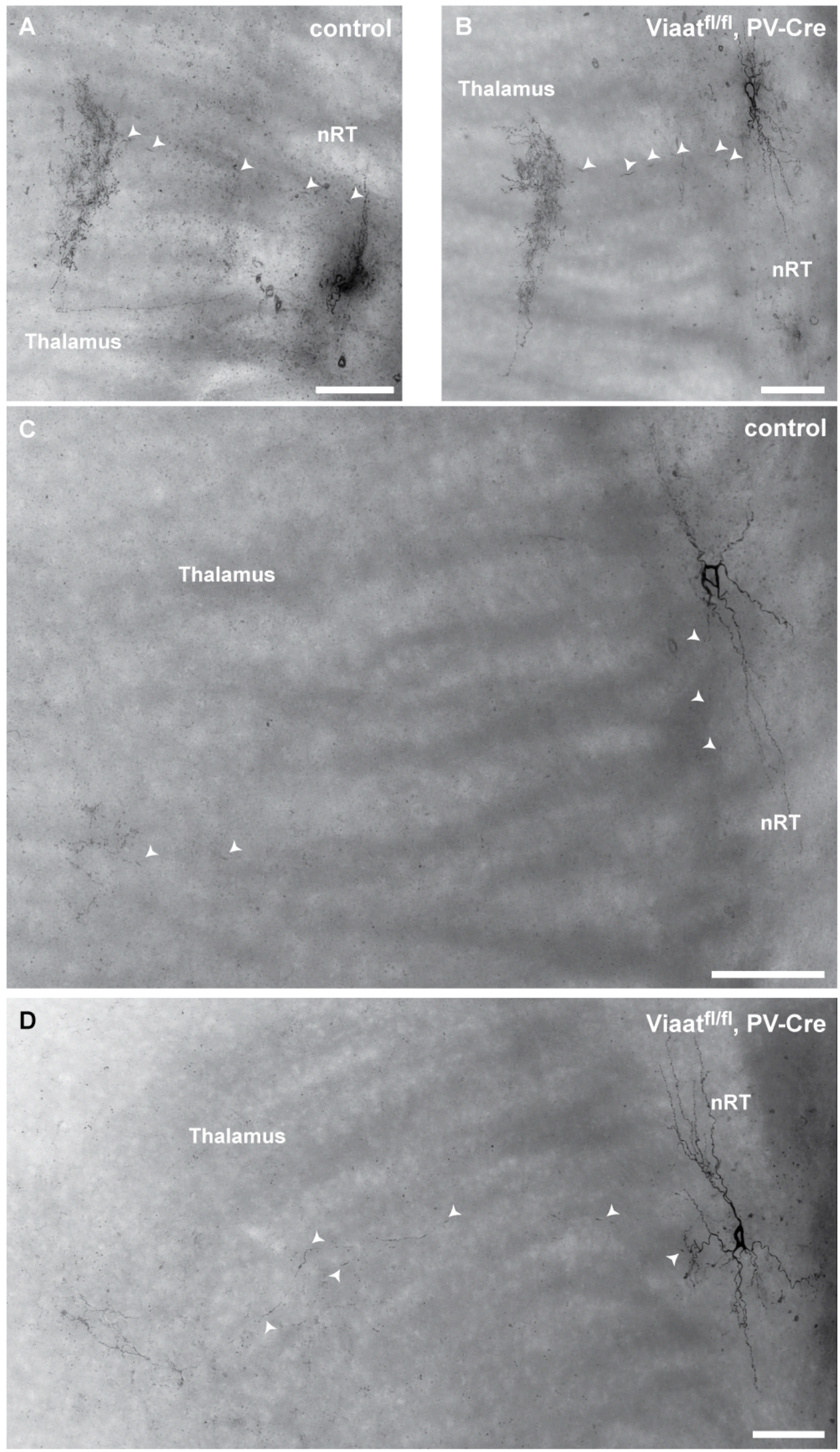


Figure 3.8: No Obvious Differences in the Axonic Morphologies of $n R T$ Neurons in Viaat ${ }^{\mathrm{fl} / \mathrm{fl}}$, PV-Cre Mice Compared to Controls

$(A, B)$ Axonal arborisation in the VPM region of the thalamus in $(A)$ control animals compared to (B) mutant animals. A compact synaptic field could be observed. (C, D) Axonal arborisations in the POM region of the thalamus of $\mathrm{nRT}$ neurons of (C) control and (D) Viaat ${ }^{f / f l}$, PV-Cre animals with a rather diffuse synaptic tuft. No obvious difference could be observed for both types of axons. White arrowheads indicate fragments of the filled and stained axon. nRT, reticular thalamic nucleus. Scale bar = $100 \mu \mathrm{m}$.

The somatodendritic region of the $\mathrm{nRT}$ neurons was analysed in more detail by reconstruction of this cellular domain with the help of the Neurolucida software (mbf Biosciences). Several contradictory studies have been published regarding a possible classification of nRT neurons, based on somatodendritic features, however, there does not appear to be any relationship between somatodendritic features and axonal morphology (Lubke, 1993; Ohara and Havton, 1996; Pinault and Deschenes, 1998; Spreafico et al., 1991). Therefore, the data of the somatodendritic characterization were pooled from cells with an axonic arborisation in the VB and POM region. No difference could be observed in the somatic features of these cells including the feret min (minimal caliper length, corresponds to minimal diameter of cell soma), feret max (longest caliper length, corresponds to the maximum diameter of the cell soma), the surface and volume of the cell body. A tendency to a higher complexity of the dendritic processes in the mutant animals could be observed. Dendritic processes seemed to cover a bigger area and also formed a bigger total volume. Furthermore the total length of the dendrites was greater in Viaat ${ }^{\mathrm{fl} / \mathrm{fl}}$, PV-Cre animals. The total number of primary dendrites was comparable between both genotypes, however the number of higher order dendrites was increased marginally, which was reflected in the increased number of branching points and total ends of dendrites. However, all these features did not show a significant difference between neurons from mutant animals and control littermates (for comparison see Table 3.1 and Figure 3.9) A more detailed analysis including the axonal processes, might reveal additional subtle changes in the cellular morphology as a result of the deletion of Viaat in these neurons. 


\begin{tabular}{|c|c|c|c|c|c|c|c|c|}
\hline & \multicolumn{3}{|c|}{ ctrl } & \multicolumn{3}{|c|}{ Viaat $^{\mathrm{f} / \mathrm{fl}}, \mathrm{PV}-\mathrm{Cre}^{+}$} & \multirow[b]{2}{*}{$p$} \\
\hline & & mean & & SEM & mean & & SEM & \\
\hline \multirow[t]{4}{*}{ Cell body } & feret Max $(\mu \mathrm{m})$ & 25,88 & \pm & 1,03 & 24,25 & \pm & 1,31 & 0,33 \\
\hline & feret Min $(\mu \mathrm{m})$ & 11,85 & \pm & 0,62 & 12,50 & \pm & 0,54 & 0,45 \\
\hline & surface $\left(\mu \mathrm{m}^{2}\right)$ & 1000,07 & \pm & 63,57 & 931,61 & \pm & 69,72 & 0,48 \\
\hline & volume $\left(\mu \mathrm{m}^{3}\right)$ & 2038,93 & \pm & 265,20 & 1911,92 & \pm & 189,26 & 0,72 \\
\hline \multirow[t]{3}{*}{ Dendrites } & surface $\left(\mu \mathrm{m}^{2}\right)$ & 5468,24 & \pm & 755,47 & 6956,95 & \pm & 1001,39 & 0,24 \\
\hline & volume $\left(\mu \mathrm{m}^{3}\right)$ & 1382,86 & \pm & 161,57 & 1818,10 & \pm & 255,66 & 0,14 \\
\hline & length $(\mu \mathrm{m})$ & 1945,44 & \pm & 313,35 & 2426,11 & \pm & 377,92 & 0,33 \\
\hline \multirow{11}{*}{$\begin{array}{l}\text { Number } \\
\text { of branches of order }\end{array}$} & 1 & 5,82 & \pm & 0,42 & 5,77 & \pm & 0,51 & 0,93 \\
\hline & 2 & 8,12 & \pm & 0,50 & 8,62 & \pm & 0,93 & 0,62 \\
\hline & 3 & 8,35 & \pm & 0,74 & 10,23 & \pm & 1,12 & 0,16 \\
\hline & 4 & 7,59 & \pm & 1,25 & 9,77 & \pm & 1,51 & 0,27 \\
\hline & 5 & 4,71 & \pm & 1,12 & 5,77 & \pm & 1,78 & 0,6 \\
\hline & 6 & 1,88 & \pm & 0,74 & 2,85 & \pm & 1,49 & 0,54 \\
\hline & 7 & 1,18 & \pm & 0,64 & 1,38 & \pm & 0,94 & 0,58 \\
\hline & 8 & 0,35 & \pm & 0,19 & 0,15 & \pm & 0,15 & 0,44 \\
\hline & nodes & 16,00 & \pm & 2,13 & 19,00 & \pm & 3,41 & 0,44 \\
\hline & ends & 22,00 & \pm & 2,25 & 25,54 & \pm & 3,63 & 0,39 \\
\hline & $\mathrm{n}$ & \multicolumn{3}{|c|}{17} & \multicolumn{3}{|c|}{13} & \\
\hline
\end{tabular}

Table 3.1: Basic Parameters of the Somatodendritic Region of Reconstructed nRT Neurons

Feret min and feret max describe the maximum and minimum diameter of the cell soma as measured with a caliper. The length describes the total length of all dendritic processes and the nodes represent branching points in the dendritic tree. The ends mark the endpoints of the highest order branch in the dendritic arbor. Values are given as mean \pm SEM, p-value calculated by Student's t-test. 

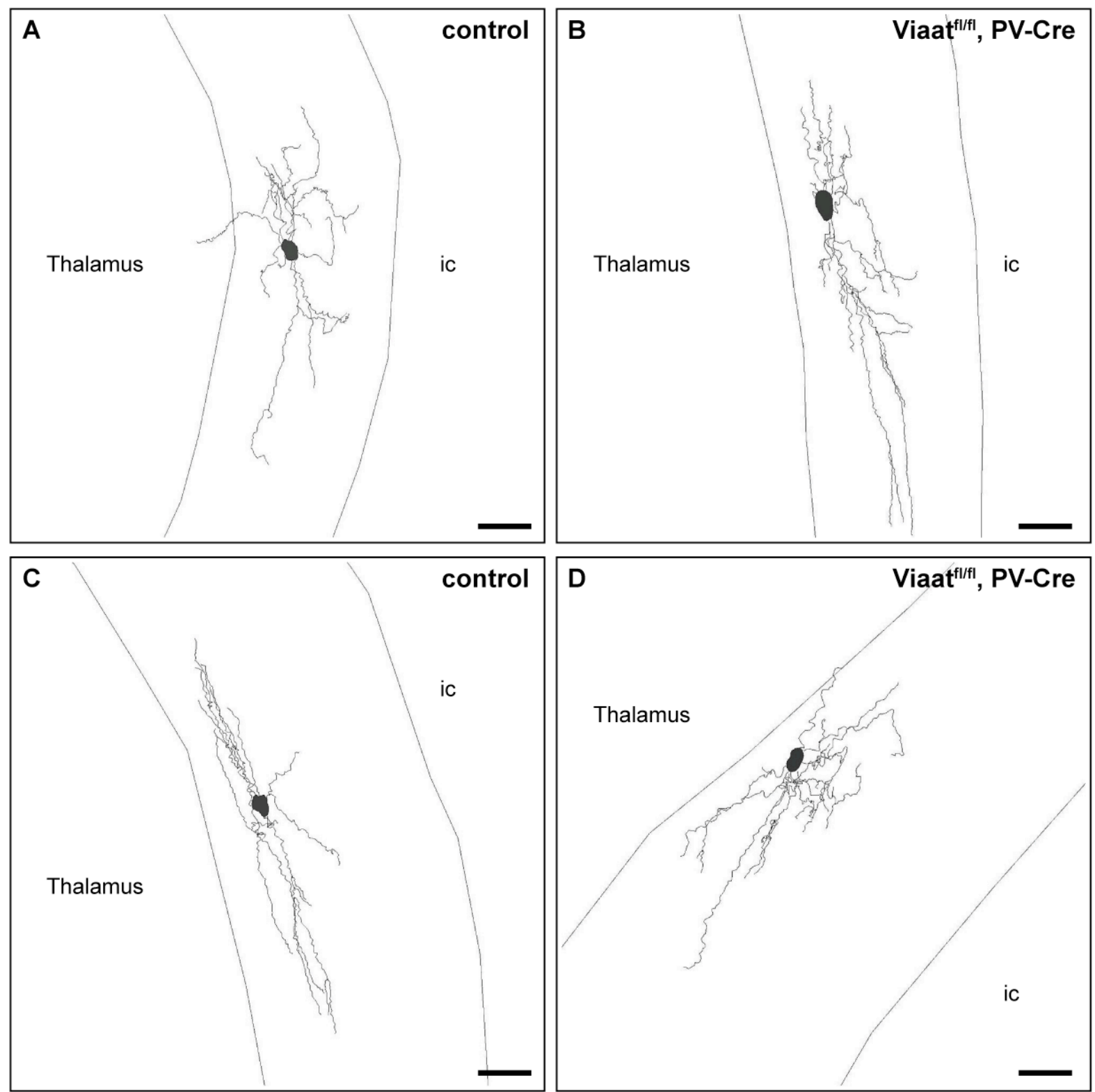

Figure 3.9: Neurolucida Reconstructions of $n R T$ Neurons of Viaat ${ }^{\mathrm{fl} / \mathrm{fl}}, \mathrm{PV}-\mathrm{Cre}$ Animals Compared to Control Mice.

Neurolucida reconstructions of the somatodentritic region of $n R T$ neurons of $(A, C)$ control animals and $(B, D)$ Viaat ${ }^{\text {fl/fl }}$, PV-Cre mice. No dramatic changes could be observed, even though a tendency to a higher complexity of neurons of mutant animals was seen. ic, internal capsule. Scale bar $=50 \mu \mathrm{m}$

\subsection{Unaltered Number of Parvalbumin Neurons}

To further analyse the consequences of the deletion of Viaat in PV-expressing cells, the number of PV positive cells was analysed. Immunostaining of coronal brain sections was performed and PV-positive cells were counted in different brain regions, including the cerebellum (Purkinje cells), nRT, cortex, hippocampus and striatum. No 
changes in the total number of PV-immunoreactive cells were detected in Viaat ${ }^{\mathrm{fl} / \mathrm{fl}}$, PV-Cre mice compared to control animals.

Thus, deletion of Viaat did not appear to elicit either loss of the affected cells, or a compensatory increase in the number of PV-positive cells.

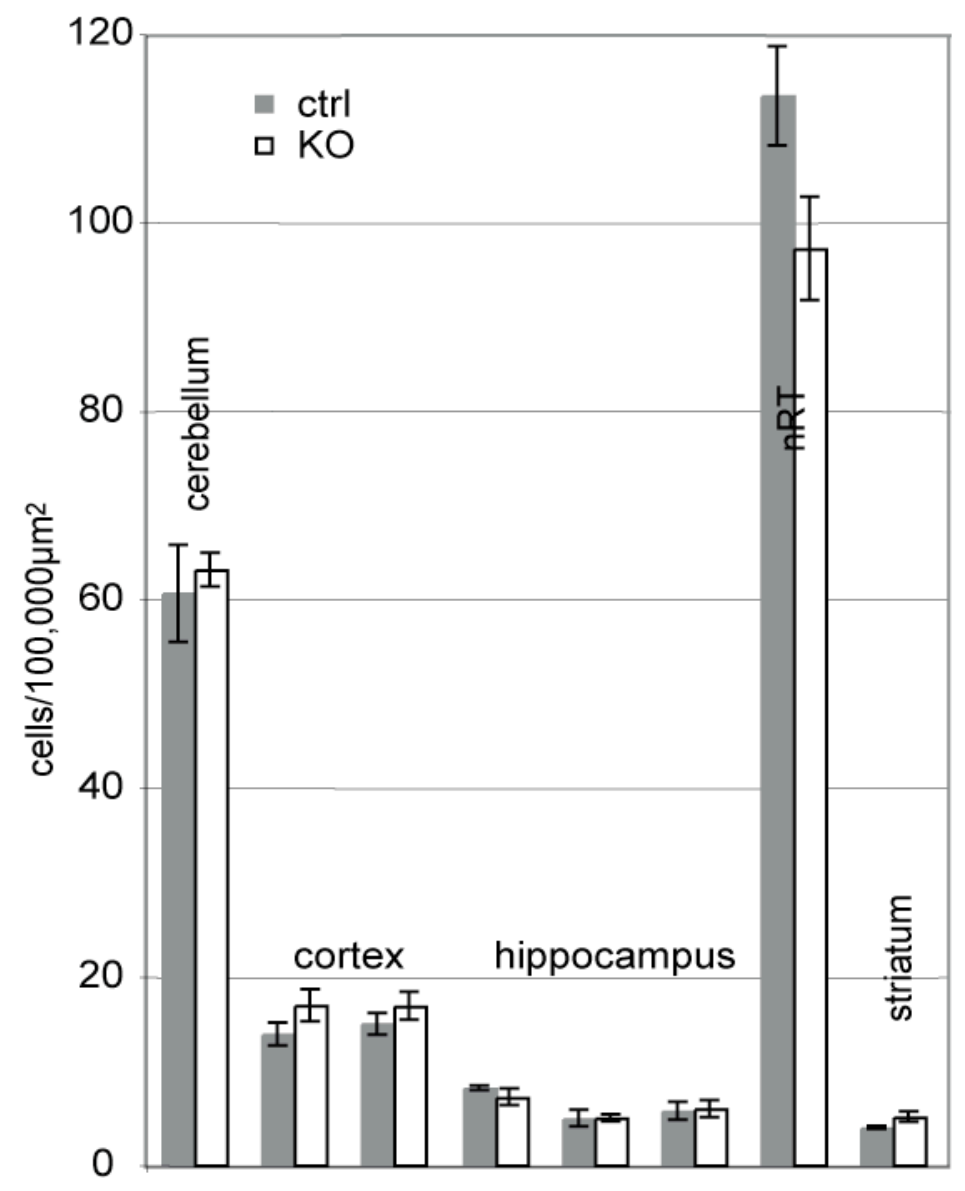

Figure 3.10: Quantification of Parvalbumin Expressing Neurons

No loss of neurons in different brain regions could be detected by quantifying PVimmunoreactive cells.

\subsection{Spontaneous Miniature IPSCs were Unaltered in the nRT of}

\section{Viaat $^{\mathrm{fl} / \mathrm{fl}}$, PV-Cre Animals}

According to the immunfluorescence analysis, the neurons of the $\mathrm{nRT}$ showed almost $100 \%$ co-labelling of PV and EGFP, and therefore Viaat should be deleted in all of the neurons of this structure in Viaat ${ }^{f / f l}, P V-C r e$ animals. The axons of the $n R T$ neurons project to the thalamus but the cells also form a connected network within the nucleus via axo-dentritic and dendrodentric synapses (Zhang and Jones, 2004 
and reviewed by Pinault, 2004). Additional GABAergic input to the $n R T$ originates in various other brain regions. In order to determine whether an alteration in GABA release within the $\mathrm{nRT}$ could be detected in Viaat ${ }^{\mathrm{fl} / \mathrm{fl}}$, PV-Cre animals, miniature IPSCs (mIPSCs) were recorded in the presence of TTX and NBQX. In the thalamocortical slice preparation used in this study, no significant change in the amplitude $\left(22.8 \pm 2.2 \mathrm{pA}\right.$ in Viaat ${ }^{\mathrm{fl} / \mathrm{fl}}$, PV-Cre compared to $20.7 \pm 2.5 \mathrm{pA}$ in control, $p=0.6)$ or frequency $(0.9 \pm 0.2 \mathrm{~Hz}$ compared to $1.2 \pm 0.2 \mathrm{~Hz}$ in control, $\mathrm{p}=0.2)$ of mIPSCs in neurons of the nRT was detected in Viaat ${ }^{f / f l}, \mathrm{PV}-\mathrm{Cre}$ animals (Figure 3.11 $B$ and $C$ ). A trend towards a lower frequency and therefore a higher inter-event interval (data not shown) was observed but did not reach statistical significance. Furthermore, the decay kinetics of the mIPSCs were not altered in Viaat ${ }^{\mathrm{fl} / \mathrm{fl}}$, PV-Cre animals compared to control animals as judged by the comparable decay time constant (8.2 $\pm 0.5 \mathrm{~ms}$ in Viaat ${ }^{\mathrm{fl} / \mathrm{l} l}$, PV-Cre cells, compared to $8.1 \pm 0.4 \mathrm{~ms}$ in control cells $p=0.9$, Figure 3.11 D). $n=13$ cells from control animals and 11 cells from Viaat $^{\mathrm{fl} / \mathrm{fl}}, \mathrm{PV}-\mathrm{Cre}$ mice.

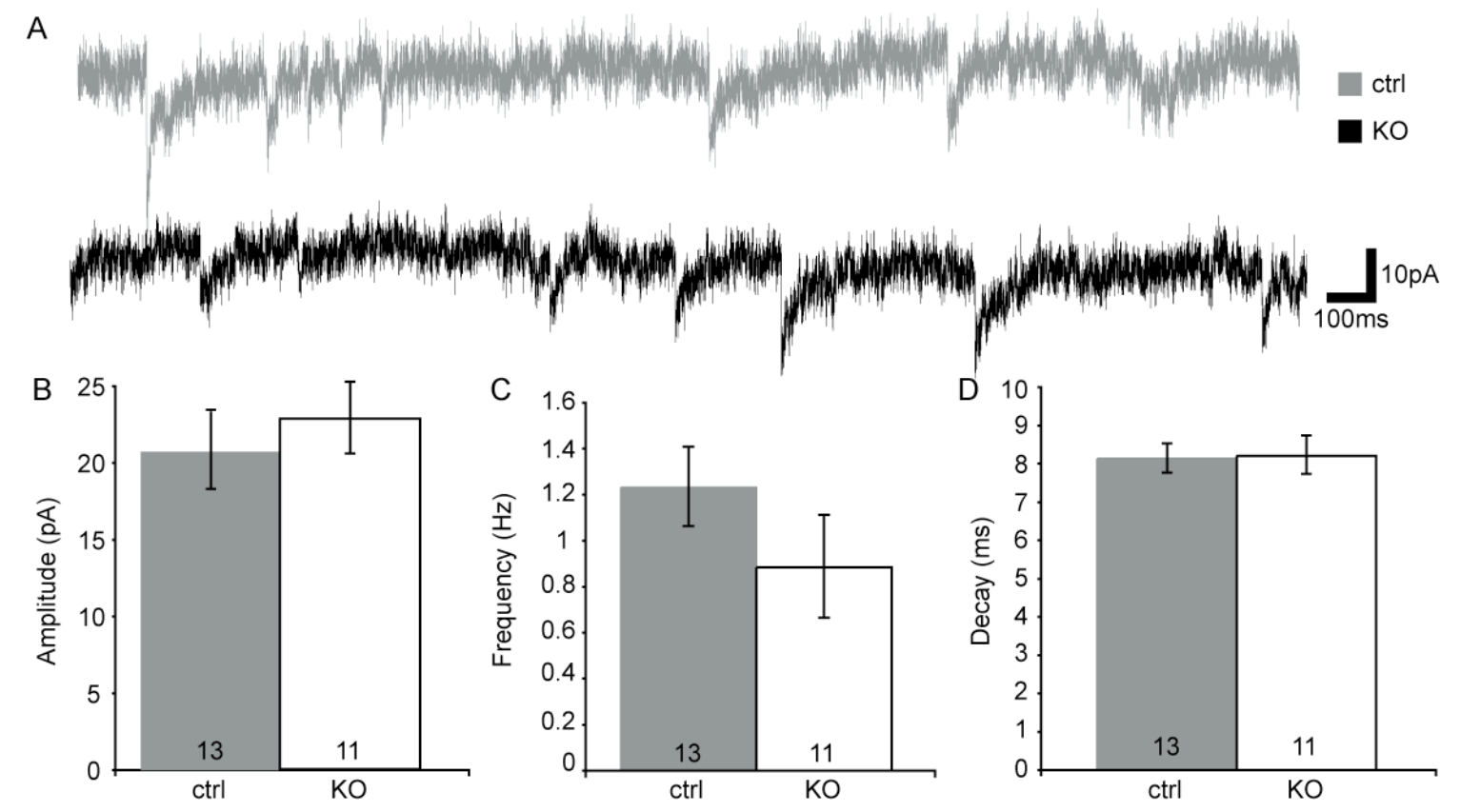

Figure 3.11: No change in mIPSC Amplitude or Frequency in the $\mathrm{nRT}$

No change in spontaneous mIPSCs was detected in neurons of the nRT (A) Raw trace of recording of mIPSCs in the presence of TTX and NBQX in Viaat ${ }^{\mathrm{fl} / \mathrm{fl}}$, PV-Cre (black) and control (grey) neurons. (B) No change of the amplitude of events was detected. (C) A trend towards a decreased frequency of mIPSCs in the Viaat ${ }^{\mathrm{fl} / \mathrm{fl}}, \mathrm{PV}$ Cre could be detected, but it was statistically not significant. (D) The decay kinetics of the events in both genotypes were identical. 


\subsection{Altered Network Oscillations in Viaat ${ }^{\mathrm{fl} / \mathrm{fl}}$, PV-Cre Animals}

Gamma oscillations are dependent on inhibitory input. In-vitro experiments showed that Kainate induced gamma oscillations can be successfully blocked by the application of GABA-receptor blocker bicucullne, but not an AMPA receptor blocker (Fisahn et al., 2004). PV-expressing GABAergic interneurons targeting the perisomatic region of pyramidal cells have been implicated in the generation, regulation and maintenance of gamma oscillations (Gulyas et al., 2010; Sohal et al., 2009). These nerve cells form highly connected networks between interneurons and principal neurons, which allows them to spread synchronous activity amongst each other, but also towards pyramidal neurons (Cobb et al., 1995). They form an abundant class of cells in the hippocampus. Furthermore, they fire phase locked, with one or two action potentials in every gamma cycle. These features put PV expressing interneurons in a position within the neuronal network to control gamma oscillations (reviewed in Bartos et al., 2007; Gulyas et al., 2010; Hajos et al., 2004). Therefore the contribution of these neurons to the generation of gamma oscillations was analysed in Viaat ${ }^{\mathrm{fl} / \mathrm{fl}}$, PV-Cre animals and control littermates.

\subsubsection{Gamma Oscillations in Submerged Conditions}

In-vitro, gamma oscillations can be induced by Kainate application and blocked by bicuculline. GABAergic inhibition is therefore necessary to induce gamma oscillations in-vitro with Kainate (Fisahn et al., 2004).

For recordings of field potentials, slices were kept in a modified submergedstyle recording chamber, with a high flow rate of the extracelluar solution. Oscillations were induced by application of $10 \mu \mathrm{M}$ Kainate via a T-valve directly onto the slice. The recording electrode was placed in the CA3 region of the hippocampus. Oscillatory activity lasted only around $16-50 \mathrm{sec}\left(35.5 \pm 4.5 \mathrm{~s}\right.$ in Viaat ${ }^{\mathrm{fl} / \mathrm{fl}}$, PV-Cre, $35.3 \pm 3.0 \mathrm{~s}$ in control), which is consistent with published data for gamma oscillations induced under submerged conditions (Gloveli et al., 2005). No difference in the gamma oscillations was detected between Viaat ${ }^{\mathrm{fl} / \mathrm{l}}$, PV-Cre and control animals. Power spectrum analysis showed no difference between the two genotypes (Figure 3.12 A). A quantification of the power of the maximum peak revealed comparable results $\left(100.1 \pm 15.9 \mu \mathrm{V}^{2} / \mathrm{Hz}\right.$ in Viaat ${ }^{\mathrm{fl} / \mathrm{fl} l}, \mathrm{PV}-\mathrm{Cre}$ compared to $100.5 \pm 20.1 \mu \mathrm{V}^{2} / \mathrm{Hz}$ in control, $\mathrm{n}$ = 28 slices form 9 mice each; Figure 3.12 B1). The frequency of the maximum peak 
was also almost identical in both genotypes $\left(32.1 \pm 0.8 \mathrm{~Hz}\right.$ in Viaat $\mathrm{f}^{\mathrm{fl} / \mathrm{fl}}$, PV-Cre compared to $32.0 \pm 0.7 \mathrm{~Hz}$ in control, $\mathrm{n}=28$ slices form 9 mice each; Figure $3.12 \mathrm{~B} 2$ ).

A

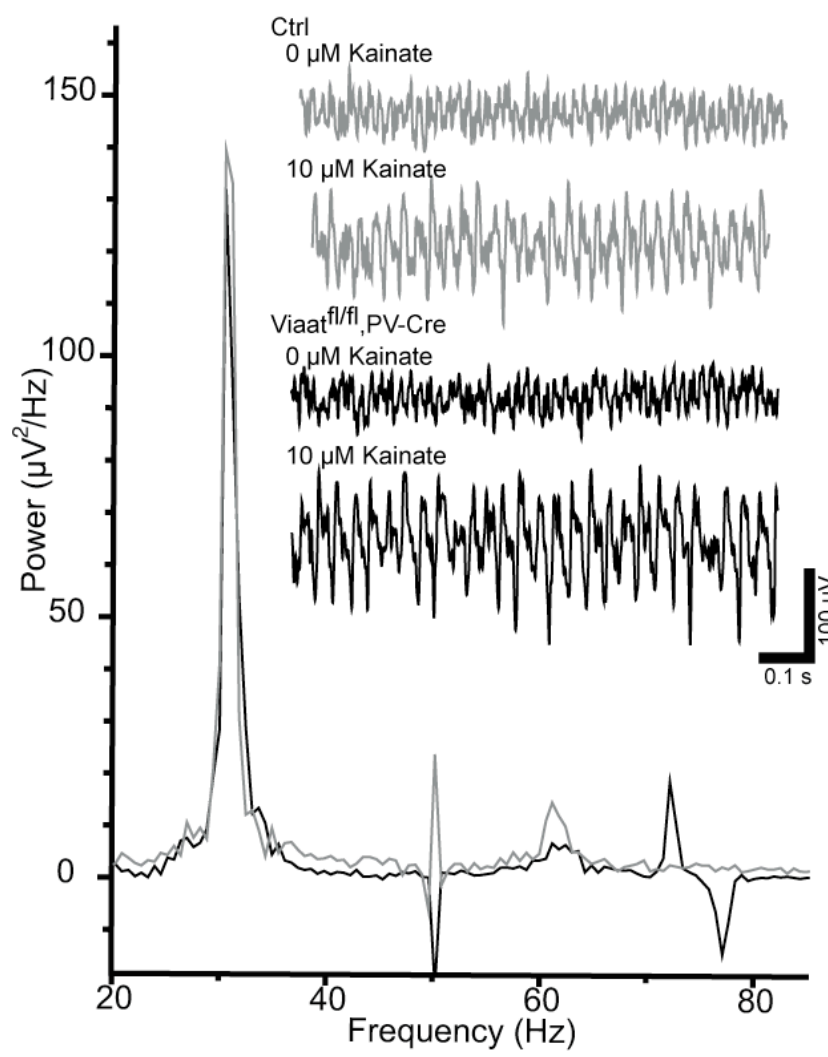

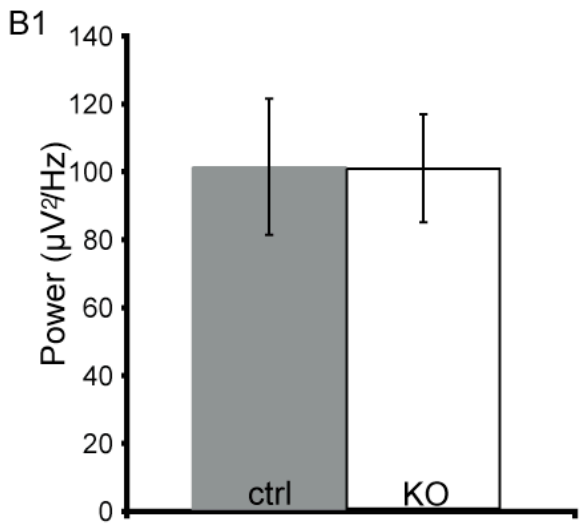

B2

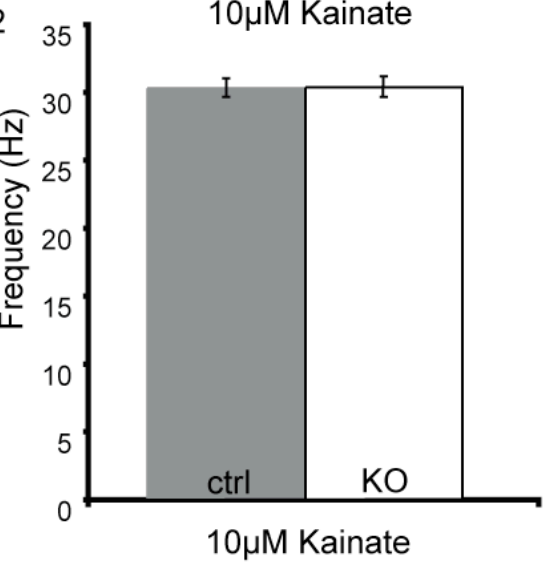

Figure 3.12: Unaltered Gamma Oscillations in Submerged Recording Conditions

(A) No difference could be detected in the gamma oscillations induced with $10 \mu \mathrm{M}$ Kainate applied via a T-valve, when recorded in submerged conditions. The trace shows a typical recording of baseline and induced gamma oscillations in Viaat ${ }^{\mathrm{flffl}}$, PVCre (black) and control (grey). The power spectrum shows one typical example from Viaat ${ }^{\mathrm{fl} / \mathrm{fl}}, \mathrm{PV}$-Cre and control. (B1) The graph shows the power of the maximum peak in the recordings in control and Viaat ${ }^{\mathrm{fl} / \mathrm{ll}}, \mathrm{PV}-\mathrm{Cre}$ animals in the presence of $10 \mu \mathrm{M}$ Kainate from 28 slices of 9 mice each. (B2) The plot depicts the frequency of the maximum power peak in the recordings from control and Viaat ${ }^{f / / f l}, \mathrm{PV}-\mathrm{Cre}$ animals, $\mathrm{n}$ $=28$ slices, 9 mice each.

\subsubsection{Gamma oscillations in Interface Conditions}

No difference between mutant and control animals could be detected in submerged conditions. However, the short duration and instability of gamma oscillations induced under submerged conditions could potentially obscure small differences. Furthermore, the submerged conditions were not optimal for establishing a dose-response curve, since under these conditions only drug application by T-valve directly onto slice resulted in a reproducible induction of gamma-oscillations, whereas 
standard application with the normal inflow system of the bath chamber was usually ineffective. Therefore, recordings of Kainate induced gamma oscillations were repeated under interface conditions. Here, the slice is kept at the interface between a flow of ACSF below and a stream of warm, moistened carbogen gas above. This setting allows an optimal supply of the slice with oxygen, which facilitates the induction and maintenance of oscillations, and keeps the recording conditions like $\mathrm{pH}$ or concentration of the drug applied more stable from experiment to experiment.

Oscillations in the gamma frequency range were induced pharmacologically by Kainate application in the ACSF solution (50 - 200nM Kainate on each slice). In this setting, oscillations could be induced within $5 \mathrm{~min}$ after the start of drug application. Long-lasting and stable rhythmic activity was induced in the hippocampal slices and recorded in the CA3b region.

Oscillatory activity could be induced with all concentrations of Kainate tested, from very small amplitudes with $50 \mathrm{nM}$ Kainate to amplitudes of up to $1 \mathrm{mV}$ with 200nM Kainate. At each concentration (50, 100 and 200nM Kainate) the power of the gamma oscillations was smaller in mutant mice compare to controls (Figure $3.13 \mathrm{~A}$ ). However, this difference reached statistical significance only for 100nM Kainate bath application. The power of the maximum peak at 100nM Kainate application was 20.4 $\pm 4.9 \mu V^{2} / \mathrm{Hz}$ in Viaat ${ }^{\mathrm{fl} / \mathrm{fl}}$, PV-Cre mutant mice ( $\mathrm{n}=25$ slice, 7 animals) compared to $60.8 \pm 10.8 \mu \mathrm{V}^{2} / \mathrm{Hz}$ in the control animals $(\mathrm{n}=38$ slices, 11 animals), $\mathrm{p}=0.005$ (see Figure $3.13 \mathrm{~A}, \mathrm{~B} 1)$. Furthermore, the frequency of the maximum peak in the power spectrum was significantly different in the mutant animals compared to the controls $(33.8 \pm 0.9 \mathrm{~Hz}$ in knockout, $30.9 \pm 0.5 \mathrm{~Hz}$ in control, $\mathrm{n}=$ see above, $\mathrm{p}=0.003$; Figure 3.13 B2).

At the highest Kainate concentration of 200nM, epileptic discharges were recorded in both genotypes (data not shown). These discharges interrupted the oscillatory activity, which resumed until the next epileptic discharge.

In spite of the fact that no obvious loss of Viaat immunoreactivity was detected in the hippocampus of Viaat ${ }^{\mathrm{fl} / \mathrm{fl}}$, PV-Cre mice, gamma oscillations in the CA3 region of the hippocampus were reduced in power while the frequency increased, which is consistent with a reduction in inhibitory drive in the Viaat ${ }^{\mathrm{fl} / f l}, \mathrm{PV}-\mathrm{Cre}$ hippocampus (Traub et al., 2000). 

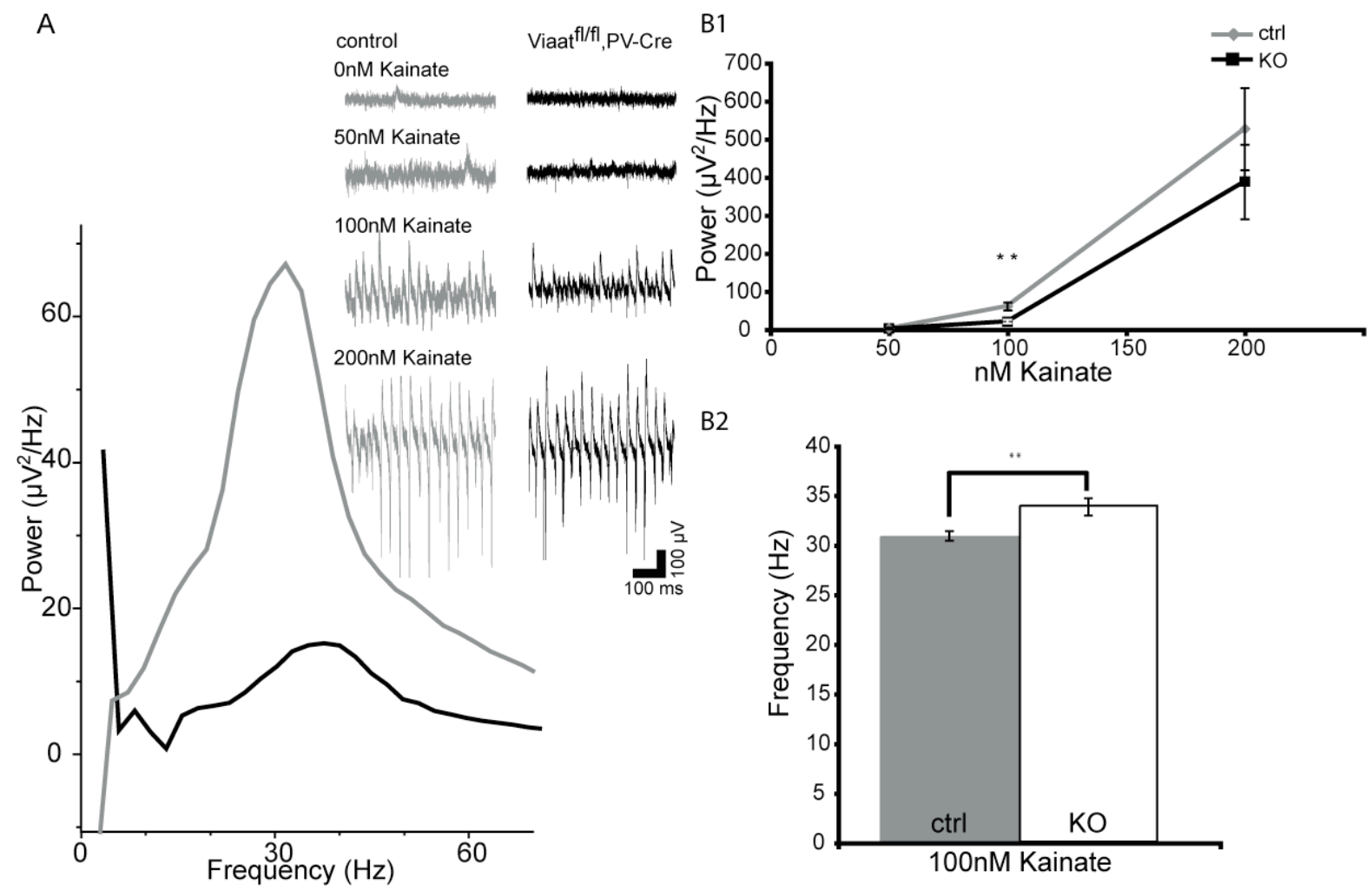

B2

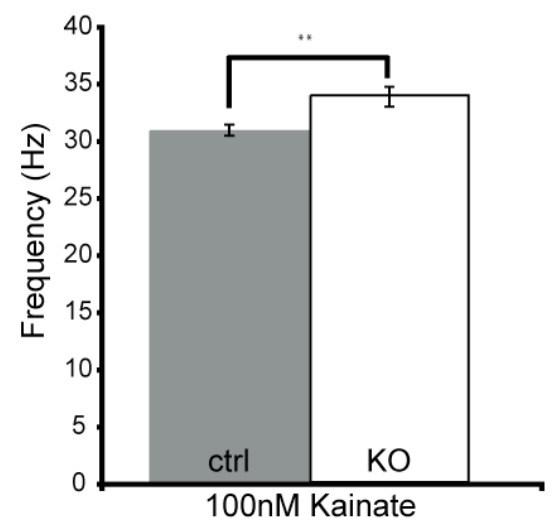

Figure 3.13: Reduced Hippocampal Gamma Oscillations in Viaat ${ }^{\mathrm{fl} / \mathrm{fl}}$, PV-Cre Mice

(A) Gamma oscillations were induced by application of different concentrations of Kainate in interface conditions. The power of the oscillations in Viaat ${ }^{f / f f l}, \mathrm{PV}-\mathrm{Cre}$ hippocampal slices was reduced for all concentrations, which can be seen in the typical traces from control (grey) and mutant (black) mice. The power spectrum shows the reduced gamma oscillation power as recorded from the CA3b region of the hippocampus in the presence of $100 \mathrm{nM}$ Kainate from mutant (black, 25 slice, 7 animals) compared to control (grey, 38 slices, 11 animals) mice. (B1) Quantification of the power of the maximum peak revealed a significant decrease in power in the mutant slice (black) compared to control (grey) animals. (B2) The mean frequency of the maximum power peak was increased in Viaat ${ }^{\mathrm{flffl}}, \mathrm{PV}$-Cre slices $(25$ slices from 7 animals) compared to control slices (36 slices from 11 animals).

\subsection{Generation of Cre-mouse Lines}

GABAergic interneurons can be classified based on different features including the expression of certain marker proteins. In order to silence different, mostly nonoverlapping subsets of GABAergic interneurons, different Cre-driver lines were developed. In these mouse lines, Cre-recombinase is expressed under the control of specific promoters for VGLUT3, Calbindin (CB) and Calretintin (CR), the expression of which defines specific subsets of interneurons. Crossing these Cre-lines with the conditional floxed Viaat mouse line allows the targeting of different GABAergic 
interneuron subsets and the analysis of their contribution to network development and activities such as network oscillations. These Cre-lines were generated either as a tamoxifen inducible or a conventional version of Cre-recombinase.

The mouse lines were generated as bacterial-artificial chromosome (BAC) transgenic animals by Dr. Wojcik. The constructs were generated by homologous recombination in E.coli (overview see Figure 3.14). The isolated and purified constructs were injected into the pronuclei of fertilised eggs by experienced staff of the animal facility of the institute. Founder animals were obtained and confirmed by PCR genotyping. Four surviving founder animals were obtained for the conventional VGLUT3-Cre line and two for the inducible Cre variant (VGLUT3-CreERT2). Six founder mice were generated for the inducible Calbindin-Cre mouse line (CBCreERT2) and seven for the inducible Calretintin-Cre mouse line (CR-CreERT2).
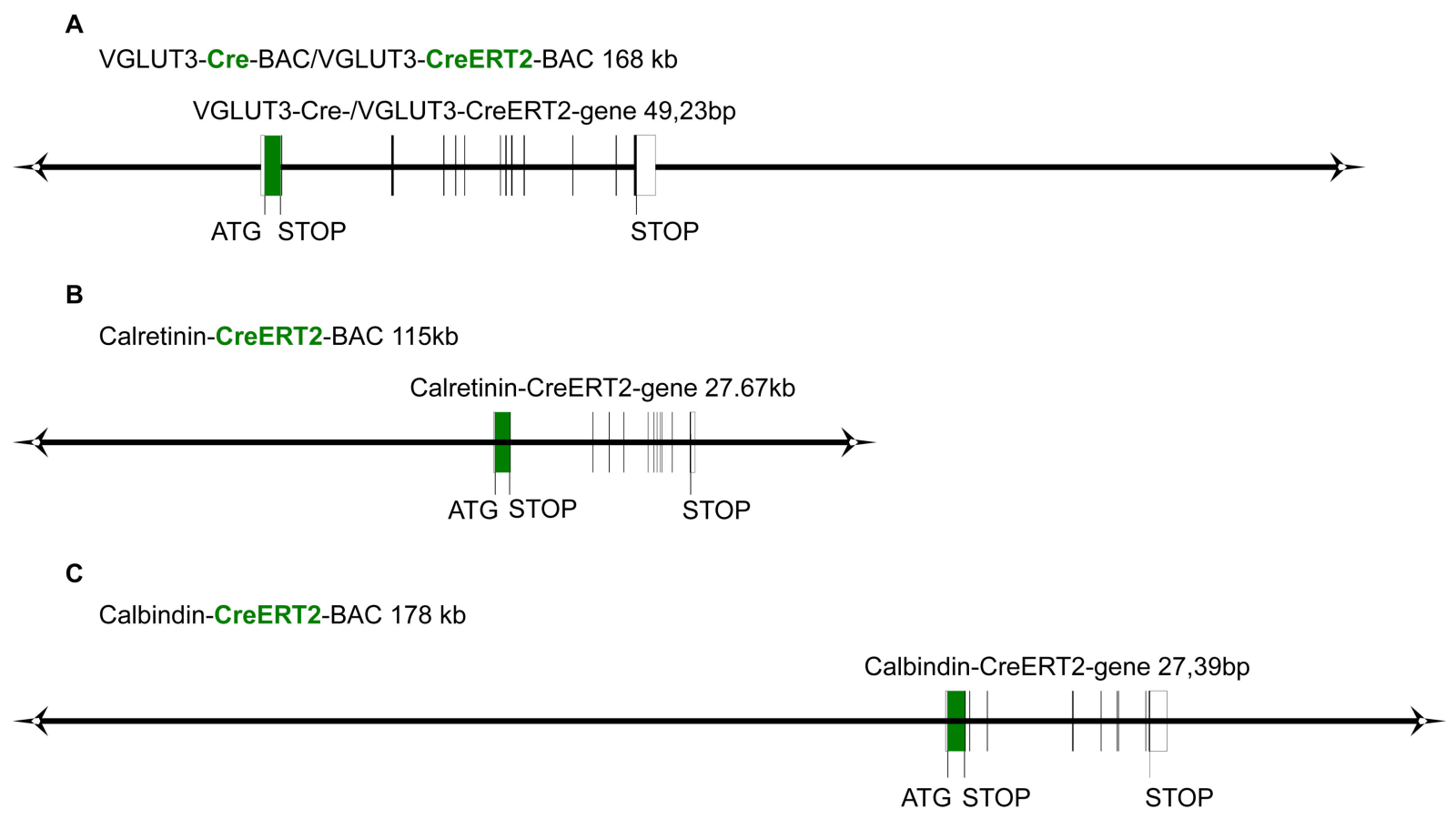

Figure 3.14: Constructs for the Generation of Cre-mouse Lines

(A) Shows the final BAC constructs for the generation of the VGLUT3-Cre mouse lines. The CreERT2 (green) or conventional Cre was introduced in frame into the start codon of the VGLUT3 gene located on the BAC (168kb) by homologous recombination in E.coli. (B) The Cre-ERT2 (green) gene was introduced into the start codon of the Calretinin gene (27.67kb), located on the $115 \mathrm{~kb}$ BAC construct. (C) The CreERT2 (green) gene was inserted into the Calbindin gene $(27.39 \mathrm{~kb})$ by recombination in E.coli, based on the 178kb BAC-construct. 


\subsection{Analysis of the Conventional VGLUT3-Cre Mouse lines}

The expression of the VGLUT3-Cre-BAC transgene was analysed for all four founder lines obtained. The different founder lines were crossed with a reporter mouse line that allowed a Cre-recombination mediated expression of EGFP. For the analysis the CAG-CAT-EGFP (CGCT) reporter mouse line was used. Double immunofluorescence labellings were performed using antibodies against VGLUT3 (red) and EGFP (green) in order to determine if the EGFP positive (Cre-positive) neurons corresponded to VGLUT3-positive neurons.

In three of four lines, EGFP expression could be observed in a pattern that largely corresponded to the published expression pattern of VGLUT3 (Herzog et al., 2004). However, the extend of reporter expression and therefore Cre-activity in the different founder lines varied considerably - it ranged from very restricted to quite widespread expression of the transgene. These differences in expression pattern were particularly apparent in the cortex and in the cerebellar Purkinje cells. Creactivity in cerebellar Purkinje cells, which transiently express VGLUT3 during development ranged from no expression in these cells (line 12) to mosaic expression of single cells or groups of Purkinje cells (Figure $3.15 \mathrm{~A}$, line 1 ) to expression in almost all cells (line 7 and 27). In the cortex, the number of EGFP expressing cells also varied considerably in the four VGLUT3-Cre lines (example of line 1 see: Figure $3.15 \mathrm{~B}$ ). Although the overall expression pattern of EGFP of three of four lines matched the published data on the expression of VGLUT3 mRNA (Fremeau et al., 2002; Gras et al., 2002; Herzog et al., 2004), co-localization of VGLUT3 and EGFP was only observed in a minority of cell somata. This was due to the fact that VGLUT3 is a synaptic protein and consequently only a minority of VGLUT3-expressing neurons had VGLUT3-positive cell bodies. This fact made the assessment of the VGLUT3-Cre expression pattern difficult. One line (VGLUT3-Cre, line 12), expressed EGFP in a pattern that was clearly much more restricted than the expression of endogenous VGLUT3. However, since reports of VGLUT3 expression in the cortex vary (Fremeau et al., 2002; Harkany et al., 2004; Herzog et al., 2004), for the remaining 3 lines it is currently unclear whether the widespread expression of EGFP in VGLUT3-Cre lines 7 and 27 is due to misexpression of the VGLUT3-Cre transgene or correctly reports transient activity of the endogenous VGLUT3-promoter in these cells. Since hippocampal VGLUT3-positive interneurons also express CCK, VGLUT3-Cre line 1 was selected for a more detailed analysis, where the distribution 
of EGFP-positive cells in the cortex and hippocampus most closely matches the published distribution of CCK-positive cortical interneurons (Freund and Buzsaki, 1996; Somogyi et al., 2004; Xu et al., 2010).

EGFP expression in interneurons of the hippocampus (Figure $3.15 \mathrm{C}$ ) was observed. The position of the EGFP-positive cells in the hippocampus matches the published localization of VGLUT3/CCK-positive GABAergic interneurons (Freund and Buzsaki, 1996; Somogyi et al., 2004), and in some EGFP-positive cell somata VGLUT3-staining was also apparent (Figure 3.15 D-F, G-I and J-L). Furthermore a promiment EGFP signal was seen in the raphe complex (Figure $3.15 \mathrm{M}$ ) and in VGLUT3-positive cholinergic interneurons in the striatum (Figure 3.15 N) (Gras et al., 2002), as well in subcortical regions including the thalamus, hypothalamus and the septum (data not shown) and the olfactory bulb (Figure 3.15 O).

The VGLUT3-Cre line 1 was crossed with Viaat ${ }^{\mathrm{fl} / \mathrm{fl}}$ mice in order to delete Viaat from to VGLUT3-expressing GABAergic neurons. 


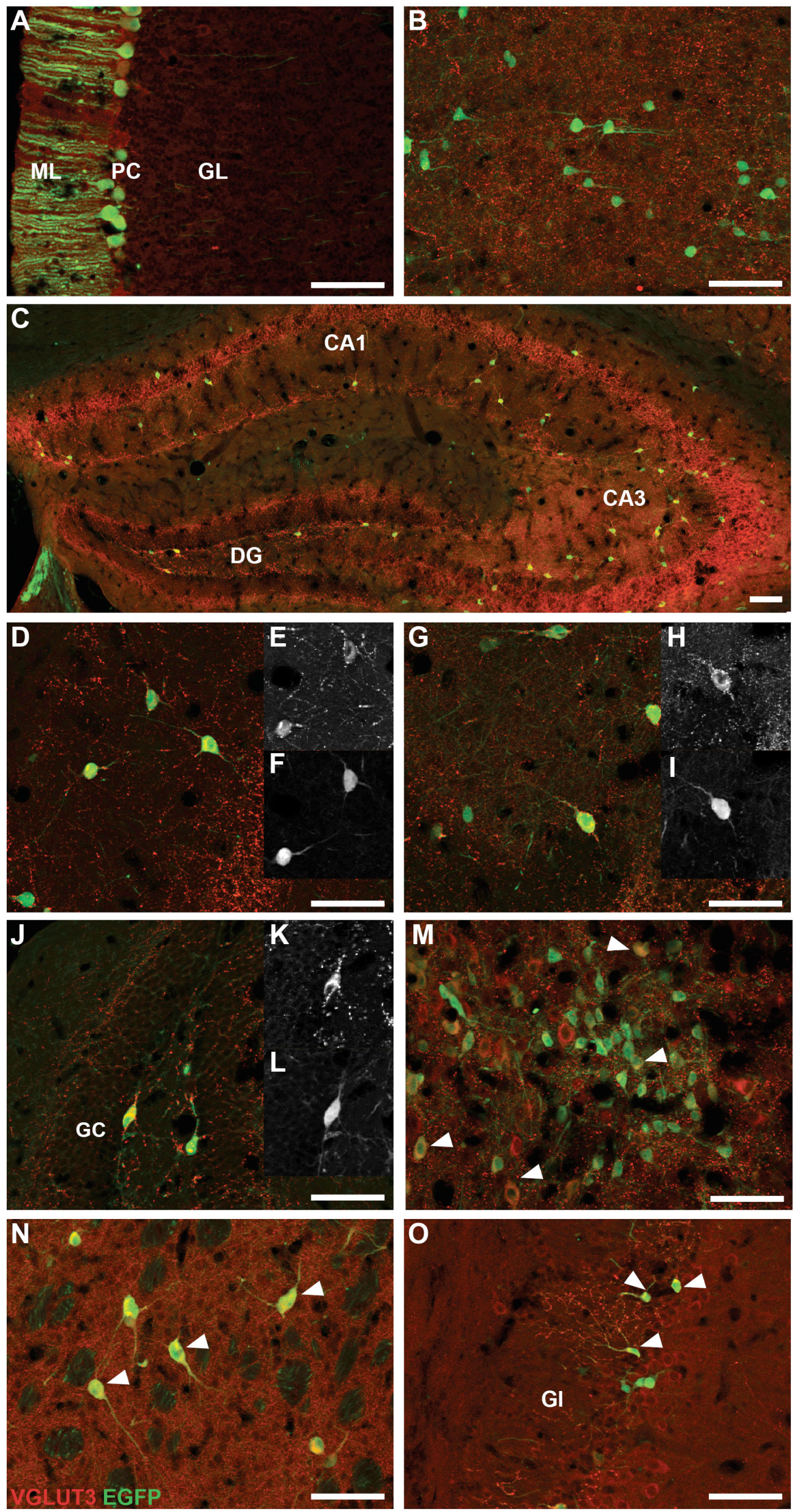


Figure 3.15: Cre-expression in Line 1 of VGLUT3-Cre ${ }^{+}, \mathrm{CGCT}^{+}$Animals, which was Used for Further Experiments

EGFP (green) expression, indicating Cre-recombinase activity, could be detected in different brain regions and cells including: $(A)$ the cerebellar Purkinje cells and $(B)$ the cortex. (C) EGFP immunoreactivity and therefore Cre activity that resembled the reported expression pattern of VGLUT3/CCK-positive neurons could be detected in the hippocampus. (D) EGFP positive cells in the CA1 region. (G) depicts VGLUT3 and EGFP positive neurons in the CA3 region. (J) Co-expression of VGLUT3 and EGFP in the DG of the hippocampus. (M) EGFP expressing neurons of the dorsal raphe nucleus. (N) Cholinergic neurons of the striatum showed Cre-activity. (O) EGFP/VGLUT3 double positive neurons in the olfactory bulb. EGFP (green) and VGLUT 3 (red) co-expression could be observed as depicted in the insets of $D, G$ and $\mathrm{J}$ (E, H, K shows VGLUT3 staining, F, I, L depicts EGFP immunoreactivity) or indicated by white arrowheads. ML, molecular layer; PC, Purkinje cell layer; GL, granule cell layer; DG, dentate gyrus; GC, granule cell layer, GI, glomerular layer. Scale $=50 \mu \mathrm{m}$.

\subsection{Analysis of Inducible Cre-mouse Lines}

Founder CreERT2-mice where crossed with the CGCT-reporter mouse line in order to visualize the CreERT2-expression pattern. Mice were treated with Tamoxifen for 10 consecutive days. 10 days after the last injection, the animals were sacrificed and analyzed by double immunofluorescent against EGFP (indicates Cre-activity) and the respective, intrinsic protein (VGLUT3, CB or CR, respectively).

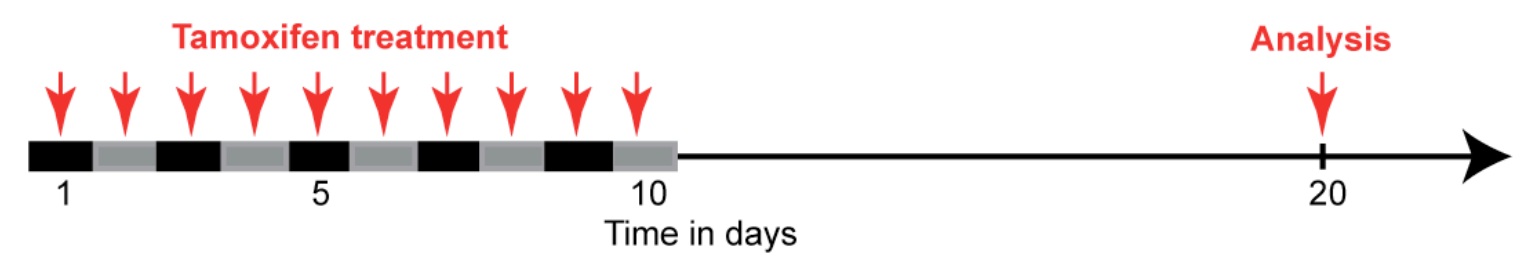

Figure 3.16: Schematic Representation of Treatment of CreERT2-mice with Tamoxifen

Induction protocol of Cre-expression with Tamoxifen injection. Mice were treated for ten consecutive days with one Tamoxifen injection. Ten days after the last injection, mice were analysed for Cre-recombinase expression.

\subsubsection{Inducible VGLUT3-CreERT2 Mouse Line}

No EGFP immunoreactivity could be detected in VGLUT3-CreERT2 transgenic mice bred from two different founder animals. 


\subsubsection{Inducible Calbindin-CreERT2 Mouse Line}

In all lines tested, EGFP immunoreactivity could be observed to different degrees in the cerebellar Purkinje cells, ranging from all Purkinje cells (including the cell body and dendrites in the molecular layer) to just single isolated cells or cell patches (Figure 3.17 A, B). Expression of the CreERT2-transgene was very limited in other brain regions. Single isolated cells expressing EGFP could be observed in cortex and hippocampus (Figure 3.17 C, D). However, with the exception of the cerebellar Purkinje cells, these cells only rarely corresponded to Calbindin-positive cells (Figure $3.17 \mathrm{C}$ ).
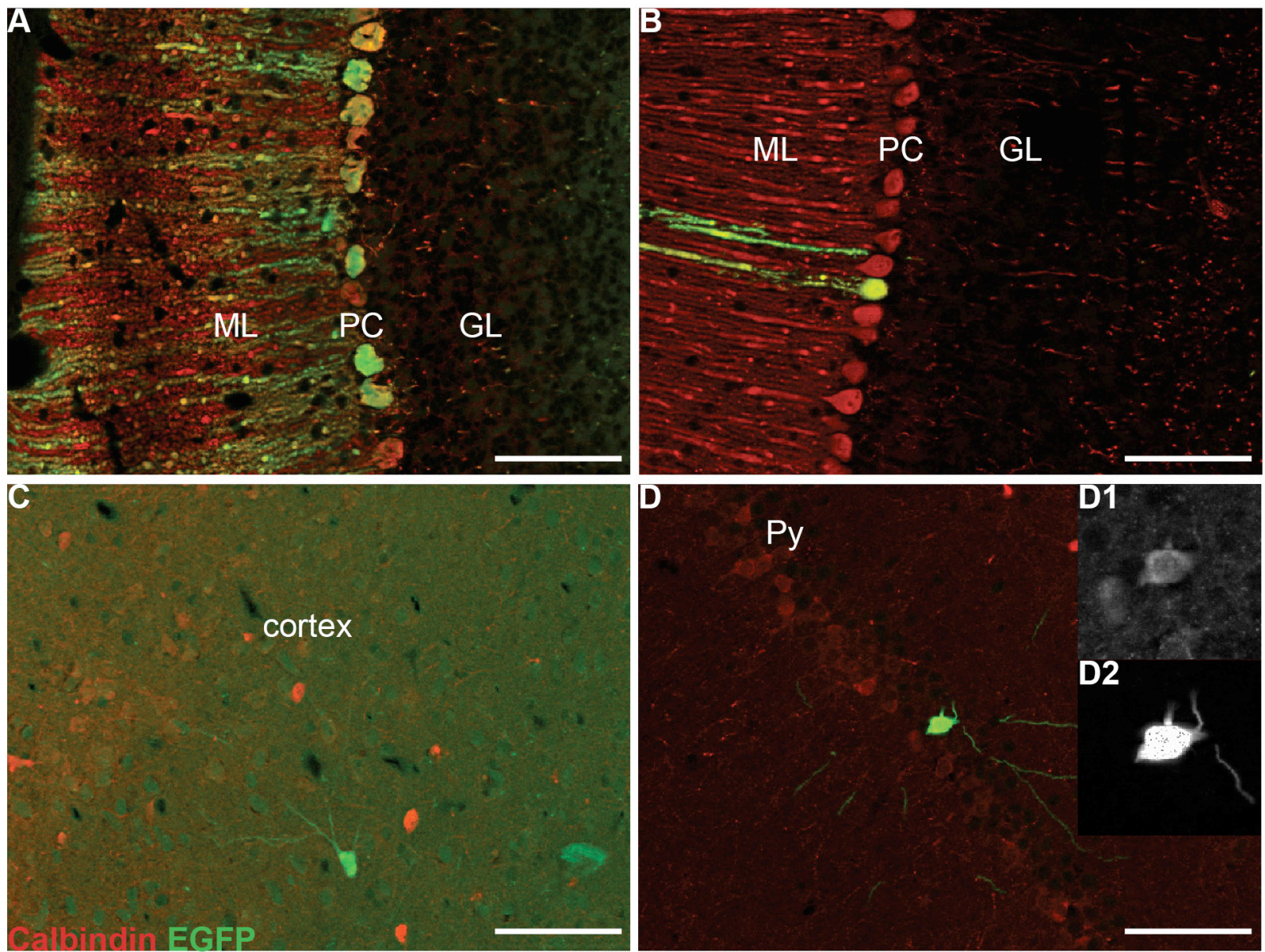

Figure 3.17: Expression of the Calbindin-CreERT2 Transgene

Expression of the CreERT2-transgene, as indicated by EGFP (green) immunoreactivity, could be observed in cerebellar Purkinje cells to different degrees - (A) all cells express EGFP as seen in line 11 or (B) just single cells (line 15). (C) Single and isolated EGFP expressing cells could be found in the cortex of line 3 and (D) hippocampus CA1 region (here line 15). Co-labelling for EGFP and CB (red) could be seen for all Purkinje cells and isolated single cells in the hippocampus (D1 CB, D2 - EGFP). ML, molecular layer; PC, Purkinje cell layer; GL, granule cell layer; Py, pyramidal cell layer. Scale: $50 \mu \mathrm{m}$ 


\subsubsection{Inducible Calretinin-CreERT2 Mouse Line}

In several founder lines, crossed to the EGFP reporter mouse line CGCT, EGFP immunoreactivity could be observed in the granule cell layer of the cerebellum (Figure $3.18 \mathrm{~A}$ ). In cortical regions single, isolated EGFP positive cells could be detected (Figure 3.18 B). Furthermore, EGFP immunoreactivity was detected in subcortical regions including the thalamus (Figure $3.18 \mathrm{C}$ ) and the hypothalamus (Figure 3.18 D). However, co-localisation of EGFP and Calretinin was rare in the cortex. In the subcortical regions a somewhat higher overlap of EGFP and Calretinin staining was observed, however, the majority of Calretinin-positive cells did not express EGFP.
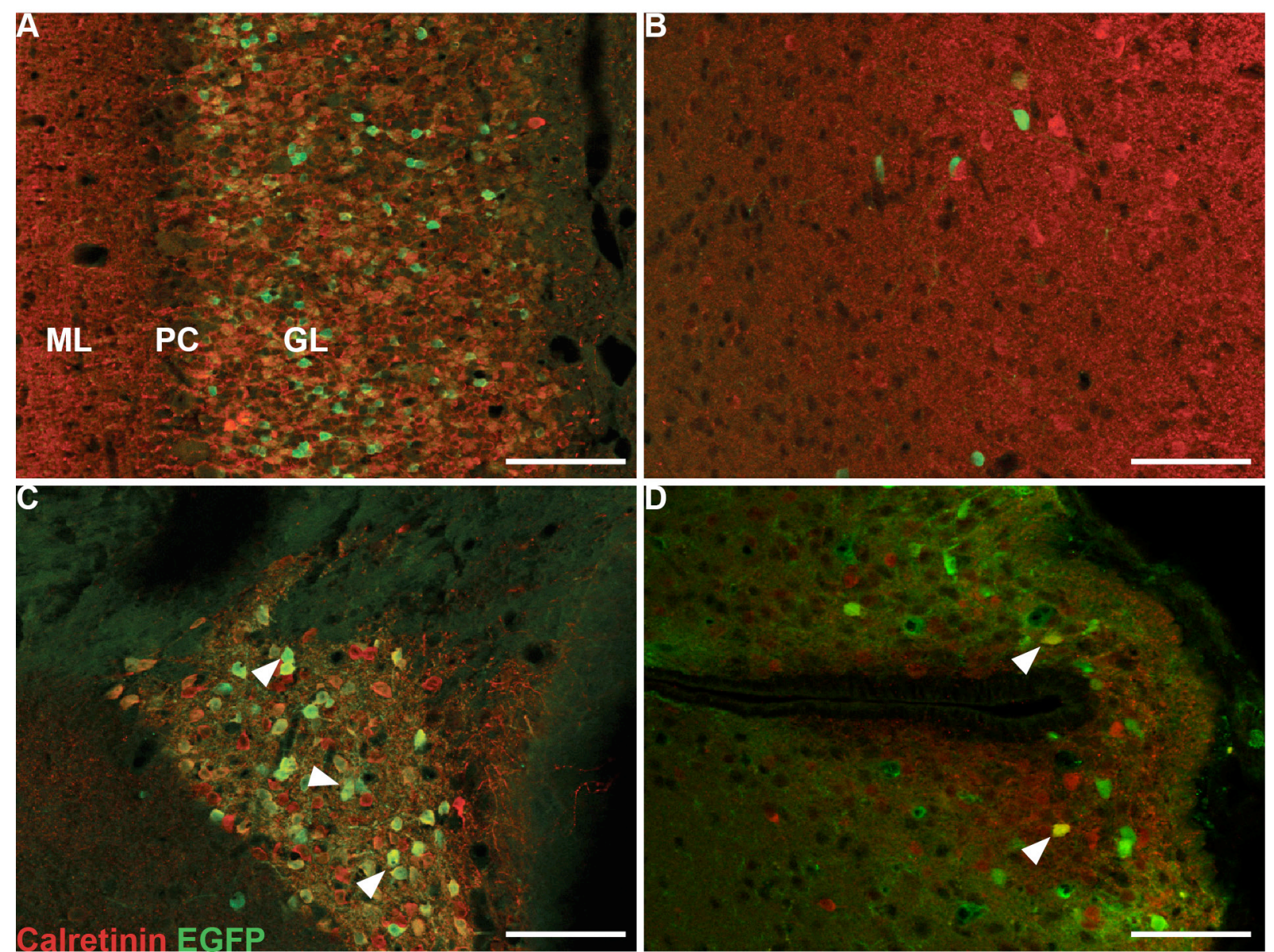

\section{Figure 3.18: Expression of the Calretinin-CreERT2 Transgene}

Expression of the CreERT2-transgene in different brain regions, including: $(A)$ the cerebellar granule cell layer and (B) single, isolated cells in the cortex. Subcortical regions like (C) the anterodorsal thalamic nucleus or (D) the hypothalamus showed expression of EGFP. Co-labelling of EGFP and Calretinin was rare in all regions analysed. Co-expression of EGFP and Calretinin is indicated with white arrowheads in the thalamus and hypothalamus. ML, molecular layer; PC, Purkinje cell layer; GL, granule cell layer. Scale $=50 \mu \mathrm{m}$. 


\subsection{Contribution of VGLUT3 Expressing Interneurons to Gamma Oscillations}

The conditional floxed Viaat mouse line was crossed to mice expressing the Cre-recombinase under the control of the VGLUT3 promoter (VGLUT3-Cre line1). This approach allows the silencing of VLGUT3 expressing GABAergic interneurons. VGLUT3 expressing interneurons in the hippocampus are a subset of CCK-positive basket cells and thus target the same perisomatic regions like PV-expressing basket cells (Freund and Katona, 2007; Somogyi et al., 2004). The contribution of these cells to the generation of gamma-oscillations remains an open question. One argument in favor of their involvement in gamma-oscillations is the fact that they express high levels of CB1-receptor and the application of CB1-receptor agonists reduces the power of gamma oscillations (Hajos et al., 2000). GABA release from VGLUT3-positive interneurons should be reduced in Viaat ${ }^{\text {fl/fl}}$, VGLUT3-Cre animals. Therefore these mice are an ideal tool to assess the contribution of the VGLUT3/CCK-expressing interneurons to the generation of gamma oscillations.

Gamma oscillations were induced by Kainate application to hippocampal slices in interface conditions. Stable and long lasting oscillatory activity could be induced with application of 100 and $200 \mathrm{nM}$ Kainate and was recorded in the CA3b region of the hippocampus. At both concentrations, the power of the gamma oscillations was smaller in mutant animals compared to control littermates. However, the difference was statistically not significant. The power of the maximum peak at $100 \mathrm{nM}$ Kainate was: $26.4 \pm 10.5 \mu \mathrm{V}^{2} / \mathrm{Hz}$ for Viaat ${ }^{\mathrm{f} / \mathrm{fl}}$, VGLUT3-Cre compared to $63.2 \pm 15.4 \mu \mathrm{V}^{2} / \mathrm{Hz}$ in controls, $p=0.053$ ( $n=22$ slices, 6 mutant animals, $n=21$ slices, 6 control animals). For $200 \mathrm{nM}$ the calculated power was: $276.1 \pm 119.7 \mu \mathrm{V}^{2} / \mathrm{Hz}$ in mutant compared to $430.2 \pm 147.9 \mu \mathrm{V}^{2} / \mathrm{Hz}, p=0.42$. $(n=21$ slices, 6 mutant animals, $n=19$ slices, 6 control animals) (Figure 3.19 A, B1).

The frequency of the maximum peak of the gamma oscillations was significantly increased for both concentrations in the Viaat ${ }^{f / f l}$, VGLUT3-Cre animals: $36.1 \pm 1.0 \mathrm{~Hz}$ in mutant mice compared to $33.2 \pm 0.7 \mathrm{~Hz}$ for controls at $100 \mathrm{nM}$ Kainate, $p=0.02$ ( $\mathrm{n}$ $=18$ slices, 6 mutant animals, $n=20$ slices, 6 control animals). At a concentration of 200nM Kainate the frequency was $32.4 \pm 0.7 \mathrm{~Hz}$ in Viaat ${ }^{\mathrm{f} / \mathrm{fl}}$, VGLUT3-Cre compared to $28.8 \pm 0.5 \mathrm{~Hz}$ in control animals, $\mathrm{p}=0.0001(\mathrm{n}=21$ slices, 6 mutant animals, $\mathrm{n}=19$ slices, 6 control animals) (Figure 3.19 B2). 
Although the reduction in the power of gamma oscillations in Viaat ${ }^{\mathrm{fl} / \mathrm{fl}}$, VGLUT3Cre hippocampal slices was not significant, the increase in frequency is reminiscent of the phenotype observed in Viaat ${ }^{\mathrm{fl} / \mathrm{fl}}$, PV-Cre slices, suggesting an involvement of VGLUT3-positive interneurons in gamma-oscillations.
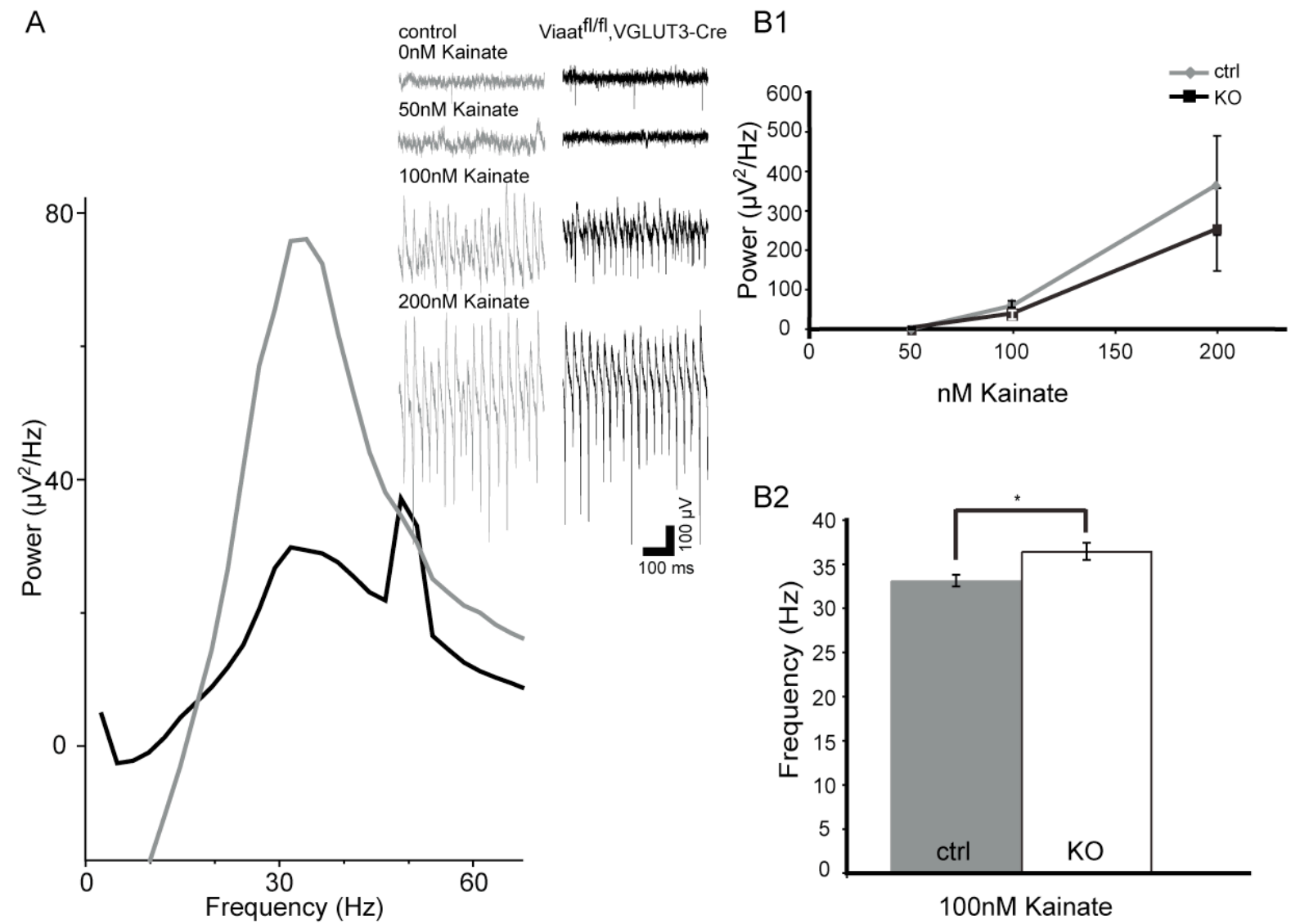

Figure 3.19: Reduced Gamma Oscillations in Viaat ${ }^{\mathrm{fl} / \mathrm{fl}}$, VGLUT3-Cre animals

(A) Gamma oscillations were induced by application of different concentrations of Kainate in interface conditions. The power of the oscillations in Viaat ${ }^{\mathrm{fl} / \mathrm{fl}}$, VGLUT3-Cre hippocampal slices was reduced for 100 and 200nM Kainate, which can be seen in the typical traces from control (grey) and mutant (black) mice. The power spectrum shows the reduced gamma oscillation power as recorded from the CA3b region of the hippocampus in the presence of 100nM Kainate from mutant (black, 21 slice, 6 animals) compared to control (grey, 22 slices, 6 animals) mice. The peak at around $50 \mathrm{~Hz}$ in the black trace results from background. It became more obvious in the lower power recordings of mutants compared to the higher power recordings of the control slices. (B1) Quantification of the power of the maximum peak revealed a decrease in power in the mutant slice (black) compared to control (grey) animals, even though it was statistically not significant. (B2) The mean frequency of the maximum power peak was increased in Viaat ${ }^{\text {fl/fl }}$, VGLUT3-Cre slices (18 slices from 6 animals) compared to control slices (20 slices from 6 animals). 


\section{Discussion}

A conditional knockout of Viaat was used to eliminate or reduce neurotransmitter release from distinct subsets of GABAergic neurons. This approach allows the analysis of inhibitory neurotransmission during postnatal development, which was not possible in the conventional Viaat knockout, because these animals die just before birth. The conditional floxed Viaat allele was combined with the transgenic expression of Cre-recombinase under the control of interneuron specific promoters. With this approach it was possible to analyse distinct, non-overlapping groups of interneurons and determine the contribution of single neuron classes to GABAergic interneuron circuits and to the formation of regulated, synchronized activity in the brain. The Cre-transgenic mouse lines used in this study express Cre under the control of the promoter for Parvalbumin (PV) and VGLUT3, respectively. These proteins characterize two distinct subsets of GABAergic interneurons that show a similar innervation pattern of postsynaptic targets. Conditional Viaat KO mice crossed with either PV-Cre or VGLUT3-Cre transgenic animals die at postnatal stages. It was therefore possible to analyze the morphological consequences of decreased inhibitory neurotransmission in the developing brain.

The contribution of the PV- and VGLUT3-expressing subsets of interneurons to the generation of oscillatory activity in the hippocampus was analysed in-vitro. PV expressing interneurons are thought to be involved in the generation of hippocampal gamma oscillations, whereas experimental evidence for the involvement of VGLUT3 positive interneurons suggests a modulatory role (Freund and Katona, 2007). In this study, the effect of PV- vs. VGLUT3-expressing interneurons on hippocampal gamma-oscillations was compared in-vitro. Gamma-oscillations were induced by the application of increasing concentrations of Kainate to horizontal hippocampal slices. In interface conditions long lasting, stable gamma oscillations could be induced with this protocol. Viaat ${ }^{\mathrm{fl} / \mathrm{fl}}$, PV-Cre slices showed a decrease of the power of gamma activity in the CA3 region by around $70 \%$ compared to control slices. Furthermore, the peak oscillatory frequency was significantly increased, which is consistent with a reduction of inhibitory input (Sohal et al., 2009; Traub et al., 2000). The results of these experiments indicate a crucial role of PV-containing GABAergic inhibitory interneurons in the generation and regulation of gamma oscillations in the hippocampus. Viaat ${ }^{\mathrm{fl} / \mathrm{fl}}$, VGLUT3-Cre animals showed a reduction of the power of 
gamma oscillations by around $60 \%$ of control levels, but the difference in power was statistically not significant. However, a significant increase of the peak gamma frequency was observed in Viaat ${ }^{\mathrm{fl} / \mathrm{fl}}$, VGLUT3-Cre animals as well. This study provides evidence that both PV- and VGLUT3-expressing interneurons, which have distinct physiological characteristics, but similar synaptic target regions, contribute to gamma oscillations. However, the exact mechanism, by which they influence oscillogenesis in the hippocampus was not analysed in the present study.

GABAergic neurotransmission is thought to be critical for the maturation of the nervous system and PV-positive neurons are an abundant class of GABAergic neurons in the brain, which also includes GABAergic projection neurons. The morphological consequences of deleting Viaat in a cell-specific manner were analysed at the single cell level in GABAergic projection neurons in Viaat ${ }^{f / / f l}$, PV-Cre animals. However, no major morphological deficits were detected in Viaat ${ }^{f / f f}, \mathrm{PV}-\mathrm{Cre}$ positive cells.

\subsection{Analysis of Cre-recombinase Expression}

\subsubsection{Expression of Cre-recombinase in the PV-Cre Mouse Line}

For the selective silencing of neurons, the Cre-loxP system was used. Since the commercially available Cre-antibody does not seem to work well enough to allow a detailed analysis of the expression pattern, we used Cre-reporter mouse lines to assess the Cre-transgene expression pattern. Cre-reporter mouse lines usually consist of a reporter allele such as the bacterial beta Galactosidase (Soriano, 1999), alkaline phosphatase (Lobe et al., 1999) or fluorescent markers like EGFP (Nakamura et al., 2006; Novak et al., 2000), whose expression is blocked by a loxPflanked stop cassette. Only after Cre-mediated removal of the stop cassette, the reporter-gene is expressed and cells and tissues where recombination took place can be detected and analysed. One commonly know problem, which was also observed in this study, is that different reporter alleles may not express equally well in all tissues and cells (Ellisor et al., 2009). Therefore, several Cre-reporter lines expressing a fluorescent marker protein were tested in this study. The Z/EG-mouse line, a double reporter line expressing beta-Galactosidase before and EGFP after Cre-mediated recombination (Novak et al., 2000), the mT-mG reporter mouse line 
expressing membrane-targeted tandem dimer Tomato (red) prior to and membrane targeted EGFP after recombination (Muzumdar et al., 2007) and a reporter line expressing nuclear-targeted Tomato following excision (Dr. M. Rossner, MPI-EM) were tested. However, none of these lines were suitable, since there was either no expression in PV-expressing cells (nuclear Tomato) or only weak expression (Z/EG) that was insufficient to visualize EGPF-positive cells in the acute slices used for electrophysiology (data not shown). The mouse line that was finally used in this study carries the CAG-CAT-EGFP (CGCT) reporter allele and expresses EGFP after Cre mediated removal of the floxed CAT stop cassette. In this line the expression of EGFP is high enough, that cells in which Cre-mediated recombination had taken place could be visualized in acute brain slices (data not shown). The CAG-CATEGFP reporter showed a more widespread expression in PV-expressing cells than the Z/EG line, where only limited EGFP-immunoreactivity could be observed in the cortex and hippocampus. On the other hand, in the cerebellum, the Z/EG allele showed a wider expression pattern, for example in the interneurons of the molecular cell layer (basket and stellate cells), which could not be observed with the CAG-CATEGFP reporter. The CAG-CAT-EGFP reporter line was selected for most of the analyses based on its high level of EGFP expression in hippocampal and cortical interneurons as well as in GABAergic projection neurons of the cerebellum and thalamus.

\subsubsection{Expression Pattern of Cre-recombinase in Calbindin-, Calretinin- and VGLUT3-Cre Mouse Lines}

In order to target further classes of GABAergic interneurons with the conditional knockout strategy used in this study, Cre-lines that express Cre-recombinase under the control of further GABAergic marker protein promoters were generated. Promoters of the calcium-binding proteins Calbindin, Calretintin and the vesicular transporter VGLUT3 were used to generate Cre-lines. The expression of these proteins marks distinct GABAergic interneuron classes (reviewed by (Freund and Buzsaki, 1996; Markram et al., 2004; McBain and Fisahn, 2001). Calbindin shows a relatively widespread expression in interneurons and is also co-expressed with PV and CCK, for example in basket cells and chandelier cells, but also in other groups. Calbindin-positive neurons therefore include, GABAergic interneurons that innervate 
somatic, dendritic or axonal regions of the interneurons and pyramidal cells ((Gulyas and Freund, 1996) reviewed in (Markram et al., 2004). By comparison, the expression of Calretinin or VGLUT3 in GABAergic interneurons is more specific to classes of cells that share common features, Calretinin expressing neurons are specialized in inhibiting other interneurons (Caputi et al., 2009; Gulyas et al., 1996), whereas VGLUT3 is expressed in a subset of CCK expressing interneurons that provide perisomatic inhibition to glutamatergic neurons in hippocampus and cortex (Somogyi et al., 2004).

The Cre-transgenic mouse lines were generated as BAC-transgenic animals. The use of the BAC technology has the advantage that the size of the BAC construct should ensure that all regulatory elements of the respective promoter are included. This should allow the correct spatial and temporal expression of the transgene. Furthermore, the large size of the BAC insulates the transgene from possible interfering chromosomal elements that might influence the expression. This should allow an expression of the Cre-recombinase that is independent of the site of integration (Yang et al., 1997). However, as shown in this study, the expression pattern of the transgene can be highly variable in the different lines ranging from no expression (VLGUT3-CreERT2 lines) to a widespread expression in different brain regions (as seen for the VGLUT3 conventional Cre-line - see Figure 3.15). The random integration of the transgene in the genome might influence its expression, despite the large size of the BAC construct. In some lines, the integrations site might not be optimal and therefore the expression did not match the endogenous expression pattern of the promoter.

The Calbindin-, Calretinin- and the VGLUT3-Cre transgenes were generated as Tamoxifen inducible version. The Cre-recombinase is fused to a mutant form of the estrogen receptor ligand-binding domain (ERT2), which prevents the nuclear localization of the construct. Only after the application of tamoxifen, the fusion protein translocates into the nucleus, where it can then perform its function by excising the floxed genomic region (Erdmann et al., 2007; Feil et al., 1997; Leone et al., 2003; Nagy, 2000). As described above (see 3.13), the expression pattern of the inducible CreERT2-variant did not match the endogenous expression of the respective protein to satisfaction. For example, in the case of the inducible VGLUT3-CreERT2 transgene, no expression at all could be detected. The CB-CreERT2 construct was reliably expressed only in the cerebellar Purkinje cells, whereas very limited 
expression was seen in all other brain regions. Similarly, the CR-CreERT2 construct, showed only limited expression in the brain. The lack of expression in most cells expressing the respective endogenous genes might be caused by incomplete induction by Tamoxifen in the brain, since the conventional VGLUT3-Cre founder lines, which are based on the same BAC as the VGLUT3-CreERT2 lines, showed a more prominent and correct expression pattern. Mice were usually injected with Tamoxifen for 10 days and the animals were sacrificed 10 days after the last injection and analysed for the expression of the CreERT2-recombinase. Further induction protocols were used in order to analyse if it might be possible to improve the recombination efficiency. Younger animals were used (starting with P14), and a shorter induction protocol (2 times for 5 consecutive days) as well the use of the active Tamoxifen component, 4-Hydroxytamoxifen, were tested. However, none of these changes in the induction protocol led to an improvement in the induction efficiency.

In some lines a restricted expression of the CreERT2-recombinase could be observed, which might be useful for the analysis of certain aspects of CreERT2expressing neurons and of the circuitry these cells are part of. For example, in Calbindin-CreERT2 line 11, CreERT2 activity could be detected solely in the cerebellar Purkinje cells. This mouse line can be used to target this class of cells in an inducible manner and for instance analyse the consequences of shutting down the output of the cerebellum.

Founder lines of the conventional VGLUT3-Cre line showed an expression of the transgene that resembled the endogenous VGLUT3 expression (see Figure 3.15) (Fremeau et al., 2002; Gras et al., 2002; Herzog et al., 2004). Therefore, VGLUT3Cre mice were crossed with Viaat ${ }^{\text {fl/fl }}$ mice to delete Viaat in VGLUT3-expressing GABAergic neurons, and the consequences for the generation of hippocampal gamma oscillations were analysed. 


\subsection{Motor - phenotype and Increased Seizure Susceptibility in Viaat $^{\mathrm{fl} / \mathrm{fl}}$, PV-Cre Animals}

Most of the analysis in the present study was performed with Viaat ${ }^{\mathrm{fl} / f l}, \mathrm{PV}-\mathrm{Cre}$ animals. PV-expressing neurons represent an abundant class of GABAergic interneurons and PV is expressed in projecting inhibitory neurons, as well.

Mice with a loss of Viaat in PV expressing neurons were born and developed normally in the first two weeks of life. Mutant animals moved less in the home cage and appeared uncoordinated compared to control littermates. Viaat ${ }^{\mathrm{fl} / \mathrm{l} l}$, PV-Cre mice showed very prominent hind limb clasping when suspended by the tail, a behaviour that was not observed in control animals. Hind limb clasping is a phenotype seen in several different mouse mutant strains. This motor-phenotype is associated with mouse models of neurodegeneration like Huntigton's Disease (Carter et al., 1999) or other neurological disorders like Rett Syndrome (Chao et al., 2010). Furthermore, mutations perturbing glycinergic neurotransmission in the brain stem and spinal cord result in hind limb clasping phenotypes (Gomeza et al., 2003; Hirzel et al., 2006). In addition, changes in GABAergic neurotransmission (Hines et al., 2008; Homanics et al., 1997) or mutations leading to cerebellar degeneration (Ivanov et al., 2004; Ribar et al., 2000) cause hind limb clasping as prominent phenotype. In Viaat ${ }^{f / / f l}$, PV-Cre mice, Cre is strongly expressed in the GABAergic cerebellar Purkinje cells (see Figure 3.4). The deletion of Viaat from these cells thus affects the main output of the cerebellum and is most likely the major cause of the hind limb clasping phenotype observed in these animals.

By three weeks of age, Viaat ${ }^{\mathrm{fl} / \mathrm{fl}}$, PV-Cre animals were prone to epileptic seizures with loss of motor control and full body stiffness in response to handling, such as providing food or water in the cage. Animals frequently did not survive these events. Epileptic seizures are another feature seen in a variety of mouse mutants. Mutations affecting every level of cellular excitability can cause epileptic phenotypes in mouse mutants (reviewed by Noebels, 2003). This includes mutations affecting ion channels (Jun et al., 1999; Lau et al., 2000), membrane receptors (Brusa et al., 1995; DeLorey et al., 1998; Homanics et al., 1997; Prosser et al., 2001; Schuler et al., 2001), proteins involved in neurotransmitter synthesis or release (Hogema et al., 2001; Kash et al., 1997; Rosahl et al., 1995), proteins responsible for the excitability of single cells as well as factors involved in regulating the formation and function of 
neuronal networks and cell survival (Acampora et al., 1996; Cobos et al., 2005; Liu et al., 2000).

In general, an imbalance of excitatory and inhibitory neurotransmission, resulting in neuronal over-excitability is regarded as a major feature of epileptic seizures. The Cre-expression pattern in Viaat ${ }^{f / / f l}$, PV-Cre animals showed a widespread expression of the recombinase in PV-positive neurons throughout the brain, including in the cerebellum, cortex, hippocampus, striatum and thalamic nuclei (see Figure 3.4). Affected neurons in these areas presumably experienced a deletion of Viaat and a subsequent reduction of synaptic GABA release, which led to an imbalance in inhibitory and excitatory neurotransmission, which most likely causes the observed seizures. However, due to the high incidence of lethal seizures in response to handling and the short life span of the Viaat ${ }^{f / f l}$, PV-Cre animals, which makes a thorough analysis of this phenotype (e.g. EEG recordings) difficult, to date, a specific brain region where the seizures might originate has not been identified.

\subsection{Loss of Viaat}

\subsubsection{Electrophysiological Evidence}

In order to show that deletion of Viaat in Viaat ${ }^{\mathrm{fl} / \mathrm{fl}}$ neurons upon expression of Cre results in a reduction of GABA neurotransmitter release, a cell culture strategy was used. Striatal neurons of Viaat ${ }^{\mathrm{fl} / \mathrm{fl}}$ animals were cultured and infected with a CreEGFP-expressing Lentivirus or an EGFP-expressing Lentivirus as control. Neuronal cultures were analysed by immunofluorescence staining and Western blotting for the expression of Viaat. In Cre-virus infected neurons, Viaat expression could not be detected after 12 days in culture. The punctate Viaat staining seen in EGFPexpressing control neurons was gone in Cre-expressing cells, and Viaat protein levels were below the detection limit of Western blot analysis. However, at 12 days in-vitro, electrophysiological analysis of autaptic neurons revealed reduced but still detectable evoked IPSCs in Cre-infected neurons, indicating the presence of Viaat molecules that could not be detected by the aforementioned techniques. After 20 days in-vitro, the majority of the Cre-infected cells showed no response or a highly reduced IPSC-amplitude in comparison to EGFP-infected control cells. These results recapitulate the results of the conventional Viaat knockout, where the majority of the 
autaptic neurons analysed are silenced and a minority of cells shows a drastic reduction of vesicular GABA release (Wojcik et al., 2006).

To analyse to which extend the GABA release is affected by the knockout of Viaat in PV-expressing cells in-vivo, mIPSCs were analysed in neurons of the $\mathrm{nRT}$. The nRT was analysed because the PV-expression starts early in this brain region at embryonic day 20 in rats (Solbach and Celio, 1991). Based on the analysis of EGFP expression in Cre-reporter mice, almost all of these cells expressed Cre in Viaat ${ }^{f / f l}, \mathrm{PV}-\mathrm{Cre}$ animals. Neurons of the nRT form a dense network of connected cells within the nucleus via dendrodentritic and axodendritic synapses and also seem to be electrically coupled by gap junctions (Cox et al., 1996; Pinault, 2004; Pinault et al., 1997; Zhang and Jones, 2004). Intrareticular inhibition is reported to be involved in the generation and regulation of sleep spindles and in the formation of hypersynchrony in absence seizures (summarized by (Fuentealba and Steriade, 2005; Steriade, 2005). However, the preparation used in this study did not reveal any reduction in amplitude or frequency of mIPSCs recorded in the presence of TTX and NBQX from nRT cells. A tendency towards a lower frequency of mIPSCs in Viaat ${ }^{\mathrm{fl} / \mathrm{fl}}$, PV-Cre animals was observed but did not reached statistical significance. These results might be due to the presence of further inhibitory input, which does not emerge from the $\mathrm{nRT}$ itself. GABAergic input into the reticular thalamic nucleus from the globus pallidus and the substantia nigra pars reticularis has been reported (Cooper and Stanford, 2002; Cornwall et al., 1990; Gandia et al., 1993; Lee and Tepper, 2007; Pare et al., 1990). This input might be sufficient to obscure a reduction in GABA release from the $\mathrm{nRT}$ neurons themselves.

As shown in the in-vitro, cell-culture based assay, it took until day in-vitro 20, until the majority of the neurons were silenced. The high seizure propensity of Viaat ${ }^{\mathrm{fl} / f l}, \mathrm{PV}-\mathrm{Cre}$ animals and the altered gamma oscillations are a clear indication that a reduction in GABA release occurs in-vivo, as well. However a partial reduction in GABA release from PV-positive neurons may be sufficient to lower the seizure threshold to the point where it becomes incompatible with survival.

\subsubsection{Immunofluorescence Analysis}

The combination of the conditional floxed Viaat mouse line with the transgenic expression of Cre-recombinase under the control of the PV promoter, allows the 
selective deletion of Viaat from PV-expressing neurons. The loss of Viaat-protein in Viaat ${ }^{f / / f l}$, PV-Cre animals compared to control littermates was investigated by immunofluorescent co-labelling of Viaat and inhibitory synaptic marker proteins like Gephyrin (Prior et al., 1992) and GAD67 (Erlander et al., 1991). The expression of Viaat was monitored in synaptic target regions affected by the genetic manipulations in the thalamus and in the cerebellum. The target region selected in the cerebellum were the cells of the deep cerebellar nuclei (DCN), which are the main cerebellar output neurons and project towards premotor areas in the thalamus and to the inferior olive (Teune et al., 1995; Voogd and Glickstein, 1998). Cells of the deep cerebellar nuclei are spontaneously firing neurons, which receive major inhibitory input from the synapses of the Purkinje cells (Aizenman and Linden, 1999; Uusisaari et al., 2007; Zheng and Raman, 2010). Immunofluorescence analysis revealed a complete loss of Viaat immunoreactivity in perisomatic Purkinje cell synapses on deep cerebellar nuclei cells (see Figure $3.5 \mathrm{~A}$ ). In the thalamus, the synaptic target regions of the $\mathrm{nRT}$ were analysed. The $\mathrm{nRT}$ consists entirely of $\mathrm{PV}$ positive GABAergic neurons, all of which also expressed Cre in Viaat ${ }^{\mathrm{fl} / \mathrm{fl}}$, PV-Cre animals (see Fig. Figure $3.4 \mathrm{C}$ ). The $\mathrm{nRT}$ forms a shell like structure on the dorsal thalamus at the interface between cortex and thalamus (reviewed in (Fuentealba and Steriade, 2005; Pinault, 2004). Neurons of the nucleus send axonal projections only towards the thalamus and not the cortex or other regions (Pinault and Deschenes, 1998). Axonal arborisations of nRT neurons are formed in nearly all thalamic nuclei, thereby inhibiting local interneurons and thalamocortical relay neurons of the thalamus. The VB region (consisting of ventral posteromedial, VPM and ventral posterolateral, VPL nucleus), which receives GABAergic input from the $\mathrm{nRT}$, showed a complete loss of Viaat immunoreactivity, confirming that deletion of the Viaat gene leads to a loss of Viaat protein in the targeted neurons, in-vivo.

Synaptic target regions of PV-positive GABAergic interneurons were analysed in the hippocampus. In this case, however, no obvious loss or reduction of Viaat immunoreactivity was detected. This may be due to the fact that PV-expressing GABAergic neurons represent only a subset of GABAergic interneurons in the hippocampus (Klausberger and Somogyi, 2008; Markram et al., 2004) and synaptic target areas of PV-positive cells are innervated by other GABAergic neurons as well. Furthermore, the expression of endogenous $\mathrm{PV}$ in GABAergic interneurons of the hippocampus and cortex starts later in development than its expression in Purkinje 
cells and in nRT neurons (del Rio et al., 1994; Solbach and Celio, 1991). Although this was not analysed in detail, this may be true for PV-Cre as well, and the deletion of the Viaat gene in interneurons may therefore occur later than in Purkinje cells and $\mathrm{nRT}$ cells, leaving less time for a reduction in Viaat protein levels prior to the death of the animals.

\subsection{No Dramatic Morphological Consequences of Viaat Deletion in PV-expressing Neurons}

The morphological consequences of the deletion of Viaat in PV expressing neurons was analysed in region with an early onset of PV expression and therefore early expression of Cre-recombinase. PV expression is reported at embryonic day 20 in the nRT and E21 in cerebellar Purkinje cells in rats but only postnatally for GABAergic interneurons of cortical and hippocampal regions (Solbach and Celio, 1991). Therefore, the DCN and the thalamus were used for an immunofluorescence analysis of synaptic markers. No obvious, dramatic morphological changes in both regions were observed when immunofluorescence labelling of synaptic marker proteins like GAD67 and 65, Synapsin or the post-synaptic marker Gephyrin were performed. However, several studies report findings that showed an influence of GABA and GABAergic neurotransmission on cell migration, neurite outgrowth and axonal patterning of inhibitory neurons (reviewed by (Represa and Ben-Ari, 2005; Sernagor et al., 2010). In-vitro studies show that trophic GABA activity influences the migration of neuroblast to their correct position in different brain structures (Behar et al., 1998; Lopez-Bendito et al., 2003). On the other hand, in-vivo studies with deletion of genes involved in GABAergic transmission like Viaat (Wojcik et al., 2006), GAD65/67 (Ji et al., 1999), KCC2 (Hubner et al., 2001) or the overexpression of KCC2 in cortical pyramidal neurons (Cancedda et al., 2007) show no obvious, overall brain histogenesis deficits. This might be explained by a compensatory mechanism of the early excitatory GABAergic signalling (for review see Ben-Ari, 2002) by other neurotransmitter systems like the glutamatergic system. However no detailed analysis of neuronal morphology was performed in these studies.

Further studies analysed the influence of GABA activity on the development of neurons in more detail and describe various effects of experimental manipulation of 
GABAergic neurotransmission. The maturation and neurite growth of cortical glutamatergic neurons seems to be dependent on the early excitatory action of GABA (Cancedda et al., 2007). An early perturbation of GABA signalling leads to a maturation defect in cortical neurons with fewer, shorter and less branched dendrites. Furthermore, Ageta-Ishihara and colleagues show that a GABA mediated increase in $\mathrm{Ca}^{2+}$ via CaMKs influences axon growth (Ageta-Ishihara et al., 2009). Cell autonomous effects on axonal development of GABAergic cells with reduced GABA synthesizing enzymes have been reported as well (Chattopadhyaya et al., 2007) The conditional knockout of GAD67 in PV-expressing cells in organotypic cortical slice cultures leads to a reduction in axonal complexity and bouton number on cortical pyramidal cells (Chattopadhyaya et al., 2007).

The Lurcher mouse mutant (Phillips, 1960) shows a virtually complete loss of all cerebellar Purkinje cells by apoptotic cell death postnatally (Caddy and Biscoe, 1979) and therefore a complete loss of the inhibitory input to the DCN. However, this loss of input leads to compensatory effects in this mouse mutant: an increase in GABA positive cells in the DCN (Sultan et al., 2002), increase of mIPSC amplitude (Linnemann et al., 2004), accumulation of $\mathrm{GABA}_{A} \alpha 1$ receptors and a decrease of Gephyrin immunoreactivity (Garin et al., 2002). A decrease of perisomatic Gephyrin (by around 10\%) and GAD67 clusters (by around 13\%) could be observed in the $\mathrm{DCN}$ of Viaat ${ }^{\mathrm{fl} / \mathrm{fl}} \mathrm{PV}$-Cre animals, as well, however these changes are less dramatic than what is observed in the Lurcher mutant. Compensatory changes in the number of PV-positive cells were not detected in Viaat ${ }^{\mathrm{flffl}}, \mathrm{PV}$-Cre brains, nor were there any major morphological alterations.

Based on analysis of biocytin-filled and partially reconstructed neurons, the axonal projections of $n R T$ neurons seem to develop normally in Viaat ${ }^{f / f / f}, \mathrm{PV}$-Cre animals. No obvious dramatic change in the axonal morphology could be observed, however, the number of synaptic boutons has not yet been analysed in detail. In Viaat ${ }^{f / f l}, \mathrm{PV}$-Cre nRT neurons, a trend towards a more complex dendritic morphology could be seen, but this increase in complexity was statistically not significant. Further detailed analyses might reveal subtle changes in the axon morphology that are not obvious with the current analysis. GABA is still produced in Viaat ${ }^{\mathrm{f} / \mathrm{fl}}, \mathrm{PV}$-Cre mice and not all GABAergic input in target areas is affected by the knockout of Viaat in PVexpressing cells. Furthermore, since the PV-Cre transgene does not show expression in all PV-positive interneurons, not all PV-expressing cells are affected by 
the knockout. A spillover of GABA released from intact GABAergic neurons or nonvesicular release (Demarque et al., 2002; Owens and Kriegstein, 2002a) might be the cause for the very mild morphological effect. Furthermore, as reported for the conventional knockout (Wojcik et al., 2006) and also seen in this study, some Viaatnegative cells still show minimal synaptic release of GABA. Such residual release might be sufficient to support neuronal maturation. The lack of major effects on cell morphology might be also due to the fact that the Viaat ${ }^{\mathrm{fl} / f l}$, PV-Cre animals die relatively soon after the onset of PV-Cre expression, which might preclude the development of potential morphological alterations due to synaptic pruning of silent synapses.

\subsection{Decreased Gamma Oscillations in Viaat ${ }^{\mathrm{fl} / \mathrm{fl}}$, PV-Cre Animals}

Deletion of Viaat from PV-expressing cells, leads to a potential reduction of GABA release of these neurons, which may have consequences for network activity such as hippocampal gamma oscillations. When gamma oscillations were induced invitro by Kainate application in acute brain slices in submerged conditions, no reduction in gamma power was seen. Under these conditions, only relatively unstable, short-lived oscillatory activity could be induced, which did not differ between mutant and control animals. However, Kainate induced gamma oscillations induced in acute brain slices in interface conditions are decreased in Viaat ${ }^{\mathrm{fl} / \mathrm{fl}}$, PV-Cre animals compared to gamma oscillations recorded in control slices. The power of gamma oscillations in the frequency range from $25-45 \mathrm{~Hz}$ is reduced by around $70 \%$ when induced with 100nM Kainate in the CA3 region. Furthermore, the frequency of the maximum peak in the calculated power spectrum is significantly increased to around $34 \mathrm{~Hz}$ in the mutant animals compared to $31 \mathrm{~Hz}$ in the controls. The increase of the frequency is in accordance with previous studies, since a decrease of inhibitory drive increases gamma frequency (Atallah and Scanziani, 2009; Mann and Mody, 2010; Traub et al., 2000). These results suggest that, although there was no obvious loss of Viaat immunoreactivity in Viaat ${ }^{f / f l}$, PV-Cre animals, GABA release from PVexpressing hippocampal interneurons was indeed reduced, and further confirms that these cells are involved in generating gamma oscillations.

The gamma oscillations that can be induced in in-vitro preparations have similarities to oscillations occurring in the gamma frequency range in-vivo (Csicsvari 
et al., 2003; Hajos and Paulsen, 2009). For instance, PV-expressing neurons in the hippocampus are involved in the generation and regulation of gamma oscillations both in-vitro (Gulyas et al., 2010) and in-vivo (Klausberger et al., 2003; Tukker et al., 2007). Perisomatic feedback inhibition has been implicated in generating gamma oscillations (Mann et al., 2005b), and thus the perisomatic innervation pattern characteristic of basket cells puts these cells in the perfect position to regulate the activity of pyramidal neurons (Freund and Katona, 2007). The highly connected network formed between PV-positive basket cells, combined with their divergent innervation of pyramidal neurons allows them to spread neuronal activity within a cellular network (Bartos et al., 2007; Cobb et al., 1995; Gulyas et al., 1999; Sik et al., 1995). Furthermore, hippocampal and cortical PV-positive basket cells fire highly phase locked to the gamma cycle, generating one or two action potentials per cycle (Gloveli et al., 2005; Gulyas et al., 2010; Hajos et al., 2004). These reports all suggest that PV-expressing basket cells play a role in the generation of regulated neuronal activity in the gamma frequency range. Somewhat surprisingly, the genetic manipulation of excitatory input to PV-positive cells reduced gamma-oscillations invitro (Fuchs et al., 2007), but not in-vivo (Racz et al., 2009), whereas a reduction the inhibitory input to these cells affected the in-vivo coupling of theta and gamma oscillations without altering the gamma oscillations themselves (Wulff et al., 2009). In contrast to this, another study showed that optogenetic up-regulation of the activity of PV-expressing cells in the cortex induces gamma oscillations, whereas inhibiting them reduces gamma oscillations (Cardin et al., 2009; Sohal et al., 2009). For all of these studies, the genetic manipulations affected all classes of PV-expressing interneurons, which most likely have distinct functions in regulating network activity. In the hippocampus, PV-expressing neurons include basket cells, axo-axonic cells, which inhibit the axon initial segment of pyramidal cells as well as bistratisfied cells and O-LM cells. The axo-axonic cells and bistratisfied cells also fire phase locked to gamma oscillations (Gloveli et al., 2005; Gulyas et al., 2010; Hajos et al., 2004), which suggests that they may contribute to the generation of gamma oscillations. OLM cells, on the other hand, do not fire locked to gamma oscillations and therefore might not contribute to the generation of gamma oscillations (Gloveli et al., 2005; Hajos et al., 2004; Tukker et al., 2007). The exact contribution of each class of PVpositive interneuron cannot be determined with our study either, since Viaat should be deleted in all classes of PV-expressing interneurons. However, the results of this 
study are in agreement with previous reports and show the significance of PVexpressing neurons in the generation of gamma oscillations.

\subsection{Reduced Gamma Oscillations in Viaat ${ }^{\mathrm{fl} / \mathrm{fl}}$, VGLUT3-Cre Animals}

Compared to the expression of PV, the expression of VGLUT3 in GABAergic interneurons is a rather specific marker for just one class of cells. VGLUT3 is expressed in a subset of CCK-positive basket cells of the hippocampus and cortex (Somogyi et al., 2004). CCK-positive basket cell are positive for either CCK/VGLUT3 or CCK/vasoactive intestinal peptide (VIP), although it is currently not clear whether VGLUT3 and VIP label two distinct groups of CCK-positive basket cells or whether these two markers might be dynamically regulated (Somogyi and Klausberger, 2005). Together with PV-expressing basket cells, CCK-expressing basket cells provide perisomatic inhibiting to pyramidal cells (Freund and Katona, 2007). In contrast to PV-positive basket cells, they have been suggested to provide a modulatory function, rather than supply the driving force for gamma oscillations (Freund and Katona, 2007). However, application of agonists of the CB1 receptor reduces the power of gamma oscillations dramatically, which can be taken as evidence for a more prominent role of the CCK basket cells, since the CB1 receptor is exclusively expressed by CCK positive cells and not by the PV containing cells (Katona et al., 1999; Marsicano and Lutz, 1999). However, the reduction in gamma power in response to $\mathrm{CB} 1$ receptor agonists might also be caused by the inhibition of glutamate release from pyramidal neurons of the hippocampus that also express the CB1 receptor (Domenici et al., 2006; Katona et al., 2006; Marsicano and Lutz, 1999). Furthermore, it has been reported that CCK containing basket cells in the hippocampus do not participate in the generation of cholinergically induced gamma oscillations in the hippocampus in-vitro (Gulyas et al., 2010). On the other hand, CCK expressing neurons participate in the regulation of oscillatory activity in-vivo (Klausberger et al., 2005). Moreover, the firing of CCK expressing interneurons is modulated by gamma activity (Tukker et al., 2007). In this study, the analysis of oscillatory activity induced by Kainate in-vitro in Viaat ${ }^{\mathrm{fl} / \mathrm{fl}}$, VGLUT3-Cre animals revealed a reduction of the power of the gamma oscillations by around $60 \%$ of wildtype levels, although the difference did not reach statistical significance. However, the frequency of the maximum peak in the gamma frequency range was significantly 
increased by around $10 \%$ in mutant animals for both concentrations of Kainate used in this study. This suggests, that CCK/VGLUT3-positive neurons indeed participate in the generation of Kainate induced gamma oscillations in-vitro. However, the exact contribution of these neurons to the generation of the oscillatory activity in the CA3 region of the hippocampus was not analysed in detail with this experiment. The changes in the maximum frequency could be due to a direct influence of the VGLUT3/CCK interneurons on the firing pattern of glutamatergic pyramidal neurons (Hajos et al., 2000). Additionally, the modulatory influence of CCK positive basket cells in the hippocampal inhibitory feedback loops (Glickfeld and Scanziani, 2006), which are important for the generation of oscillations (Csicsvari et al., 2003; Mann et al., 2005a; Mann et al., 2005b), could be responsible for the observed phenotype. Although a direct influence of CCK-positive basket cells, at least for cholinergically induced in-vitro gamma oscillations, has not been reported (Gulyas et al., 2010), an indirect influence of the CCK expressing neurons via synaptic contacts to PV expressing neurons is possible (Karson et al., 2009). In addition, these neurons might exert their regulatory function in the hippocampus by integrating several subcortical inputs and might thereby fine-regulate the overall oscillatory activity in the hippocampus (reviewed by Freund and Katona, 2007). With the Viaat ${ }^{\mathrm{flfl}}$, VGLUT3Cre mouse model used in this study, the remaining questions with regard to the contribution of CCK/VGLUT3-positive basket cells to network function can be analysed in more detail and possibly answered. 


\section{Summary and Conclusions}

GABA is the main inhibitory neurotransmitter in the mature central nervous system. It is thought to play an important role as a trophic factor in the development of single neurons and neuronal circuits. Furthermore, inhibitory input of different types of GABAergic interneurons to dendritic and axonic processes of principal, glutamatergic neurons is essential for the fine tuning of the input/output behaviour of these cells. Interneurons are involved in the modulation of neuronal excitability, the integration and generation of temporally synchronized oscillations among bigger groups of neurons, which are vital for higher brain functions like learning or memory.

Therefore, the conditional silencing of distinct subtypes of GABAergic neurons allows the analysis of the contribution of these classes of neurons to GABAergic neurotransmission. A conditional floxed Viaat mouse line was crossed with mouse lines expressing Cre-recombinase under the control of the PV and VGLUT3 promoter, which allows the deletion of Viaat in these two distinct, non-overlapping classes of neurons.

The morphological consequences of reduced GABAergic neurotransmission was analysed in Viaat ${ }^{\mathrm{fl} / \mathrm{fl}}, \mathrm{PV}-\mathrm{Cre}$ animals. However, no dramatic alteration could be observed in the Viaat ${ }^{\mathrm{f} / \mathrm{fl}}$, PV-Cre animals. Based on immunofluorescence analysis of the deep cerebellar nuclei and the thalamus, axonic and dendritic processes including synaptic contacts seemed to have developed normally. The analysis of single biocytin-filled nRT neurons did not reveal any dramatic morphological deficits as a result of the genetic deletion of Viaat in these neurons.

The contribution of PV- and VGLUT3-positive GABAergic interneurons to oscillogenesis in the hippocampus was analysed. Kainate induced gamma oscillations in the CA3 region of the hippocampus were recorded in slices from Viaat ${ }^{\mathrm{fl} / f l}, \mathrm{PV}-\mathrm{Cre}$ and Viaat ${ }^{\mathrm{fl} / \mathrm{fl}}$, VGLUT3-Cre animals. The power of gamma oscillations induced in-vitro was reduced up to $70 \%$ in Viaat ${ }^{f l f l}$, PV-Cre slices compared to controls. The peak frequency was significantly increased in these slices due to the decreased inhibitory drive in the hippocampus. This underlined the significance of PV-expressing hippocampal interneurons in the generation of gamma oscillations. Viaat $^{f / f \mid}$, VGLUT3-Cre animals showed a reduction in the power gamma oscillations induced in-vitro by around $60 \%$ compared to controls, which was however statistically not significant. The frequency of the maximum gamma peak in the field recordings 
was significantly increased. This provided evidence for a direct contribution of VGLUT3-positive basket cells to the generation of Kainate induced gamma oscillations in-vitro.

Thus, both PV-positive and VGLUT3-positive basket cells, which have distinct physiological properties, but similar morphological features, contribute to the generation of gamma oscillations in the hippocampus in-vitro. A more detailed analysis of the VGLUT3-positive basket cells in particular, should provide further insight into how these cells participate in rhythmic network activity. 


\section{References}

Acampora, D., Mazan, S., Avantaggiato, V., Barone, P., Tuorto, F., Lallemand, Y., Brulet, P., and Simeone, A. (1996). Epilepsy and brain abnormalities in mice lacking the Otx1 gene. Nat Genet 14, 218-222.

Acsady, L., Arabadzisz, D., and Freund, T.F. (1996). Correlated morphological and neurochemical features identify different subsets of vasoactive intestinal polypeptideimmunoreactive interneurons in rat hippocampus. Neuroscience 73, 299-315.

Ageta-Ishihara, N., Takemoto-Kimura, S., Nonaka, M., Adachi-Morishima, A., Suzuki, K., Kamijo, S., Fujii, H., Mano, T., Blaeser, F., Chatila, T.A., et al. (2009). Control of cortical axon elongation by a GABA-driven $\mathrm{Ca} 2+$ /calmodulin-dependent protein kinase cascade. J Neurosci 29, 13720-13729.

Agmon, A., and Connors, B.W. (1991). Thalamocortical responses of mouse somatosensory (barrel) cortex in vitro. Neuroscience 41, 365-379.

Aizenman, C.D., and Linden, D.J. (1999). Regulation of the rebound depolarization and spontaneous firing patterns of deep nuclear neurons in slices of rat cerebellum. $J$ Neurophysiol 82, 1697-1709.

Akbarian, S., Kim, J.J., Potkin, S.G., Hagman, J.O., Tafazzoli, A., Bunney, W.E., Jr., and Jones, E.G. (1995). Gene expression for glutamic acid decarboxylase is reduced without loss of neurons in prefrontal cortex of schizophrenics. Arch Gen Psychiatry 52, 258-266.

Antonopoulos, J., Pappas, I.S., and Parnavelas, J.G. (1997). Activation of the GABAA receptor inhibits the proliferative effects of bFGF in cortical progenitor cells. Eur J Neurosci 9, 291-298.

Ascoli, G.A., Alonso-Nanclares, L., Anderson, S.A., Barrionuevo, G., BenavidesPiccione, R., Burkhalter, A., Buzsaki, G., Cauli, B., Defelipe, J., Fairen, A., et al. (2008). Petilla terminology: nomenclature of features of GABAergic interneurons of the cerebral cortex. Nat Rev Neurosci 9, 557-568.

Atallah, B.V., and Scanziani, M. (2009). Instantaneous modulation of gamma oscillation frequency by balancing excitation with inhibition. Neuron 62, 566-577.

Bartos, M., Vida, I., and Jonas, P. (2007). Synaptic mechanisms of synchronized gamma oscillations in inhibitory interneuron networks. Nat Rev Neurosci 8, 45-56.

Bauer, M., Oostenveld, R., Peeters, M., and Fries, P. (2006). Tactile spatial attention enhances gamma-band activity in somatosensory cortex and reduces low-frequency activity in parieto-occipital areas. J Neurosci 26, 490-501.

Behar, T.N., Schaffner, A.E., Scott, C.A., O'Connell, C., and Barker, J.L. (1998). Differential response of cortical plate and ventricular zone cells to GABA as a migration stimulus. J Neurosci 18, 6378-6387. 
Belforte, J.E., Zsiros, V., Sklar, E.R., Jiang, Z., Yu, G., Li, Y., Quinlan, E.M., and Nakazawa, K. (2010). Postnatal NMDA receptor ablation in corticolimbic interneurons confers schizophrenia-like phenotypes. Nat Neurosci 13, 76-83.

Belmonte, M.K., Cook, E.H., Jr., Anderson, G.M., Rubenstein, J.L.R., Greenough, W.T., Beckel-Mitchener, A., Courchesne, E., Boulanger, L.M., Powell, S.B., Levitt, P.R., et al. (2004). Autism as a disorder of neural information processing: directions for research and targets for therapy[ast]. Mol Psychiatry 9, 646-663.

Ben-Ari, Y. (2001). Cell death and synaptic reorganizations produced by seizures. Epilepsia 42, 5-7.

Ben-Ari, Y. (2002). Excitatory actions of gaba during development: the nature of the nurture. Nat Rev Neurosci 3, 728-739.

Ben-Ari, Y. (2008a). GABA: Role in Neuronal Circuit Development. Book: The new enceyclopedia of Neuroscience (NRSC), 12.

Ben-Ari, Y. (2008b). Neuro-archaeology: pre-symptomatic architecture and signature of neurological disorders. Trends Neurosci 31, 626-636.

Ben-Ari, Y., Cherubini, E., Corradetti, R., and Gaiarsa, J.L. (1989). Giant synaptic potentials in immature rat CA3 hippocampal neurones. J Physiol 416, 303-325.

Ben-Ari, Y., Gaiarsa, J.L., Tyzio, R., and Khazipov, R. (2007). GABA: a pioneer transmitter that excites immature neurons and generates primitive oscillations. Physiol Rev 87, 1215-1284.

Bouilleret, V., Loup, F., Kiener, T., Marescaux, C., and Fritschy, J.M. (2000). Early loss of interneurons and delayed subunit-specific changes in GABA(A)-receptor expression in a mouse model of mesial temporal lobe epilepsy. Hippocampus 10, 305-324.

Bouyer, J.J., Montaron, M.F., and Rougeul, A. (1981). Fast fronto-parietal rhythms during combined focused attentive behaviour and immobility in cat: cortical and thalamic localizations. Electroencephalogr Clin Neurophysiol 51, 244-252.

Brovelli, A., Lachaux, J.P., Kahane, P., and Boussaoud, D. (2005). High gamma frequency oscillatory activity dissociates attention from intention in the human premotor cortex. Neuroimage 28, 154-164.

Brusa, R., Zimmermann, F., Koh, D.S., Feldmeyer, D., Gass, P., Seeburg, P.H., and Sprengel, R. (1995). Early-onset epilepsy and postnatal lethality associated with an editing-deficient GluR-B allele in mice. Science 270, 1677-1680.

Buckmaster, P.S., and Jongen-Relo, A.L. (1999). Highly specific neuron loss preserves lateral inhibitory circuits in the dentate gyrus of kainate-induced epileptic rats. J Neurosci 19, 9519-9529.

Buzsaki, G., and Draguhn, A. (2004). Neuronal oscillations in cortical networks. Science 304, 1926-1929. 
Buzsaki, G., Horvath, Z., Urioste, R., Hetke, J., and Wise, K. (1992). High-frequency network oscillation in the hippocampus. Science 256, 1025-1027.

Buzsaki, G., Leung, L.W., and Vanderwolf, C.H. (1983). Cellular bases of hippocampal EEG in the behaving rat. Brain Res 287, 139-171.

Caddy, K.W., and Biscoe, T.J. (1979). Structural and quantitative studies on the normal $\mathrm{C} 3 \mathrm{H}$ and Lurcher mutant mouse. Philos Trans R Soc Lond B Biol Sci 287, 167-201.

Cancedda, L., Fiumelli, H., Chen, K., and Poo, M.M. (2007). Excitatory GABA action is essential for morphological maturation of cortical neurons in vivo. J Neurosci 27, 5224-5235.

Caputi, A., Rozov, A., Blatow, M., and Monyer, H. (2009). Two calretinin-positive GABAergic cell types in layer $2 / 3$ of the mouse neocortex provide different forms of inhibition. Cereb Cortex 19, 1345-1359.

Cardin, J.A., Carlen, M., Meletis, K., Knoblich, U., Zhang, F., Deisseroth, K., Tsai, L.H., and Moore, C.I. (2009). Driving fast-spiking cells induces gamma rhythm and controls sensory responses. Nature 459, 663-667.

Carter, R.J., Lione, L.A., Humby, T., Mangiarini, L., Mahal, A., Bates, G.P., Dunnett, S.B., and Morton, A.J. (1999). Characterization of progressive motor deficits in mice transgenic for the human Huntington's disease mutation. J Neurosci 19, 3248-3257.

Celio, M.R. (1990). Calbindin D-28k and parvalbumin in the rat nervous system. Neuroscience 35, 375-475.

Chao, H.T., Chen, H., Samaco, R.C., Xue, M., Chahrour, M., Yoo, J., Neul, J.L., Gong, S., Lu, H.C., Heintz, N., et al. (2010). Dysfunction in GABA signalling mediates autism-like stereotypies and Rett syndrome phenotypes. Nature 468, 263-269.

Chattopadhyaya, B., Di Cristo, G., Wu, C.Z., Knott, G., Kuhlman, S., Fu, Y., Palmiter, R.D., and Huang, Z.J. (2007). GAD67-mediated GABA synthesis and signaling regulate inhibitory synaptic innervation in the visual cortex. Neuron 54, 889-903.

Chaudhry, F.A., Reimer, R.J., Bellocchio, E.E., Danbolt, N.C., Osen, K.K., Edwards, R.H., and Storm-Mathisen, J. (1998). The vesicular GABA transporter, VGAT, localizes to synaptic vesicles in sets of glycinergic as well as GABAergic neurons. J Neurosci 18, 9733-9750.

Chavas, J., and Marty, A. (2003). Coexistence of excitatory and inhibitory GABA synapses in the cerebellar interneuron network. J Neurosci 23, 2019-2031.

Christensen, H., and Fonnum, F. (1991). Uptake of glycine, GABA and glutamate by synaptic vesicles isolated from different regions of rat CNS. Neurosci Lett 129, 217220.

Clayton, G.H., Owens, G.C., Wolff, J.S., and Smith, R.L. (1998). Ontogeny of cationCl- cotransporter expression in rat neocortex. Brain Res Dev Brain Res 109, 281292. 
Cobb, S.R., Buhl, E.H., Halasy, K., Paulsen, O., and Somogyi, P. (1995). Synchronization of neuronal activity in hippocampus by individual GABAergic interneurons. Nature 378, 75-78.

Cobos, I., Calcagnotto, M.E., Vilaythong, A.J., Thwin, M.T., Noebels, J.L., Baraban, S.C., and Rubenstein, J.L. (2005). Mice lacking Dlx1 show subtype-specific loss of interneurons, reduced inhibition and epilepsy. Nat Neurosci 8, 1059-1068.

Cohen, I., Navarro, V., Clemenceau, S., Baulac, M., and Miles, R. (2002). On the origin of interictal activity in human temporal lobe epilepsy in vitro. Science 298, 1418-1421.

Connors, B.W., and Gutnick, M.J. (1990). Intrinsic firing patterns of diverse neocortical neurons. Trends Neurosci 13, 99-104.

Cooper, A.J., and Stanford, I.M. (2002). Calbindin D-28k positive projection neurones and calretinin positive interneurones of the rat globus pallidus. Brain Res 929, 243251.

Cornwall, J., Cooper, J.D., and Phillipson, O.T. (1990). Projections to the rostral reticular thalamic nucleus in the rat. Exp Brain Res 80, 157-171.

Cossart, R., Bernard, C., and Ben-Ari, Y. (2005). Multiple facets of GABAergic neurons and synapses: multiple fates of GABA signalling in epilepsies. Trends Neurosci 28, 108-115.

Cossart, R., Dinocourt, C., Hirsch, J.C., Merchan-Perez, A., De Felipe, J., Ben-Ari, Y., Esclapez, M., and Bernard, C. (2001). Dendritic but not somatic GABAergic inhibition is decreased in experimental epilepsy. Nat Neurosci 4, 52-62.

Cox, C.L., Huguenard, J.R., and Prince, D.A. (1996). Heterogeneous axonal arborizations of rat thalamic reticular neurons in the ventrobasal nucleus. $\mathrm{J}$ Comp Neurol 366, 416-430.

Coyle, J.T., Tsai, G., and Goff, D. (2003). Converging evidence of NMDA receptor hypofunction in the pathophysiology of schizophrenia. Ann N Y Acad Sci 1003, 318327.

Csicsvari, J., Jamieson, B., Wise, K.D., and Buzsaki, G. (2003). Mechanisms of gamma oscillations in the hippocampus of the behaving rat. Neuron 37, 311-322.

del Rio, J.A., de Lecea, L., Ferrer, I., and Soriano, E. (1994). The development of parvalbumin-immunoreactivity in the neocortex of the mouse. Brain Res Dev Brain Res 81, 247-259.

DeLorey, T.M., Handforth, A., Anagnostaras, S.G., Homanics, G.E., Minassian, B.A., Asatourian, A., Fanselow, M.S., Delgado-Escueta, A., Ellison, G.D., and Olsen, R.W. (1998). Mice lacking the beta3 subunit of the GABAA receptor have the epilepsy phenotype and many of the behavioral characteristics of Angelman syndrome. J Neurosci 18, 8505-8514. 
Demarque, M., Represa, A., Becq, H., Khalilov, I., Ben-Ari, Y., and Aniksztejn, L. (2002). Paracrine intercellular communication by a Ca2+- and SNARE-independent release of GABA and glutamate prior to synapse formation. Neuron 36, 1051-1061.

Dichter, M.A., and Ayala, G.F. (1987). Cellular mechanisms of epilepsy: a status report. Science 237, 157-164.

Domenici, M.R., Azad, S.C., Marsicano, G., Schierloh, A., Wotjak, C.T., Dodt, H.U., Zieglgansberger, W., Lutz, B., and Rammes, G. (2006). Cannabinoid receptor type 1 located on presynaptic terminals of principal neurons in the forebrain controls glutamatergic synaptic transmission. J Neurosci 26, 5794-5799.

Drake, C.T., and Milner, T.A. (2002). Mu opioid receptors are in discrete hippocampal interneuron subpopulations. Hippocampus 12, 119-136.

Dumoulin, A., Rostaing, P., Bedet, C., Levi, S., Isambert, M.F., Henry, J.P., Triller, A., and Gasnier, B. (1999). Presence of the vesicular inhibitory amino acid transporter in GABAergic and glycinergic synaptic terminal boutons. J Cell Sci 112 ( Pt 6), 811-823.

Ellisor, D., Koveal, D., Hagan, N., Brown, A., and Zervas, M. (2009). Comparative analysis of conditional reporter alleles in the developing embryo and embryonic nervous system. Gene Expr Patterns 9, 475-489.

Erdmann, G., Schutz, G., and Berger, S. (2007). Inducible gene inactivation in neurons of the adult mouse forebrain. BMC Neurosci 8, 63.

Erlander, M.G., Tillakaratne, N.J., Feldblum, S., Patel, N., and Tobin, A.J. (1991). Two genes encode distinct glutamate decarboxylases. Neuron 7, 91-100.

Esclapez, M., Hirsch, J.C., Ben-Ari, Y., and Bernard, C. (1999). Newly formed excitatory pathways provide a substrate for hyperexcitability in experimental temporal lobe epilepsy. J Comp Neurol 408, 449-460.

Fan, J., Byrne, J., Worden, M.S., Guise, K.G., McCandliss, B.D., Fossella, J., and Posner, M.I. (2007). The relation of brain oscillations to attentional networks. J Neurosci 27, 6197-6206.

Feil, R., Wagner, J., Metzger, D., and Chambon, P. (1997). Regulation of Cre recombinase activity by mutated estrogen receptor ligand-binding domains. Biochem Biophys Res Commun 237, 752-757.

Fernandez-Chacon, R., and Sudhof, T.C. (1999). Genetics of synaptic vesicle function: toward the complete functional anatomy of an organelle. Annu Rev Physiol 61, 753-776.

Ferrarelli, F., Huber, R., Peterson, M.J., Massimini, M., Murphy, M., Riedner, B.A., Watson, A., Bria, P., and Tononi, G. (2007). Reduced sleep spindle activity in schizophrenia patients. Am J Psychiatry 164, 483-492.

Ferrarelli, F., Massimini, M., Peterson, M.J., Riedner, B.A., Lazar, M., Murphy, M.J., Huber, R., Rosanova, M., Alexander, A.L., Kalin, N., and Tononi, G. (2008). Reduced evoked gamma oscillations in the frontal cortex in schizophrenia patients: a TMS/EEG study. Am J Psychiatry 165, 996-1005. 
Ferrarelli, F., Peterson, M.J., Sarasso, S., Riedner, B.A., Murphy, M.J., Benca, R.M., Bria, P., Kalin, N.H., and Tononi, G. (2010). Thalamic dysfunction in schizophrenia suggested by whole-night deficits in slow and fast spindles. Am J Psychiatry 167, 1339-1348.

Ferrarelli, F., and Tononi, G. (2011). The thalamic reticular nucleus and schizophrenia. Schizophr Bull 37, 306-315.

Fisahn, A. (2005). Kainate receptors and rhythmic activity in neuronal networks: hippocampal gamma oscillations as a tool. J Physiol 562, 65-72.

Fisahn, A., Contractor, A., Traub, R.D., Buhl, E.H., Heinemann, S.F., and McBain, C.J. (2004). Distinct roles for the kainate receptor subunits GluR5 and GluR6 in kainate-induced hippocampal gamma oscillations. J Neurosci 24, 9658-9668.

Fisahn, A., Pike, F.G., Buhl, E.H., and Paulsen, O. (1998). Cholinergic induction of network oscillations at $40 \mathrm{~Hz}$ in the hippocampus in vitro. Nature 394, 186-189.

Fisahn, A., Yamada, M., Duttaroy, A., Gan, J.W., Deng, C.X., McBain, C.J., and Wess, J. (2002). Muscarinic induction of hippocampal gamma oscillations requires coupling of the M1 receptor to two mixed cation currents. Neuron 33, 615-624.

Foldy, C., Lee, S.H., Morgan, R.J., and Soltesz, I. (2010). Regulation of fast-spiking basket cell synapses by the chloride channel CIC-2. Nat Neurosci 13, 1047-1049.

Ford, J.M., Mathalon, D.H., Whitfield, S., Faustman, W.O., and Roth, W.T. (2002). Reduced communication between frontal and temporal lobes during talking in schizophrenia. Biol Psychiatry 51, 485-492.

Frassoni, C., Bentivoglio, M., Spreafico, R., Sanchez, M.P., Puelles, L., and Fairen, A. (1991). Postnatal development of calbindin and parvalbumin immunoreactivity in the thalamus of the rat. Brain Res Dev Brain Res 58, 243-249.

Fremeau, R.T., Jr., Burman, J., Qureshi, T., Tran, C.H., Proctor, J., Johnson, J., Zhang, H., Sulzer, D., Copenhagen, D.R., Storm-Mathisen, J., et al. (2002). The identification of vesicular glutamate transporter 3 suggests novel modes of signaling by glutamate. Proc Natl Acad Sci U S A 99, 14488-14493.

Fremeau, R.T., Jr., Kam, K., Qureshi, T., Johnson, J., Copenhagen, D.R., StormMathisen, J., Chaudhry, F.A., Nicoll, R.A., and Edwards, R.H. (2004). Vesicular glutamate transporters 1 and 2 target to functionally distinct synaptic release sites. Science 304, 1815-1819.

Freund, T.F. (2003). Interneuron Diversity series: Rhythm and mood in perisomatic inhibition. Trends Neurosci 26, 489-495.

Freund, T.F., and Buzsaki, G. (1996). Interneurons of the hippocampus. Hippocampus 6, 347-470.

Freund, T.F., and Katona, I. (2007). Perisomatic inhibition. Neuron 56, 33-42.

Fries, P. (2009). Neuronal gamma-band synchronization as a fundamental process in cortical computation. Annu Rev Neurosci 32, 209-224. 
Fries, P., Nikolic, D., and Singer, W. (2007). The gamma cycle. Trends Neurosci 30, 309-316.

Fries, P., Reynolds, J.H., Rorie, A.E., and Desimone, R. (2001). Modulation of oscillatory neuronal synchronization by selective visual attention. Science 291, 15601563.

Fritschy, J.M., and Brunig, I. (2003). Formation and plasticity of GABAergic synapses: physiological mechanisms and pathophysiological implications. Pharmacol Ther 98, 299-323.

Frotscher, M., and Zimmer, J. (1983). Lesion-Induced Mossy Fibers to the Molecular Layer of the Rat Fascia Dentata - Identification of Postsynaptic Granule Cells by the Golgi-Em Technique. J Comp Neurol 215, 299-311.

Fuchs, E.C., Zivkovic, A.R., Cunningham, M.O., Middleton, S., Lebeau, F.E., Bannerman, D.M., Rozov, A., Whittington, M.A., Traub, R.D., Rawlins, J.N., and Monyer, H. (2007). Recruitment of parvalbumin-positive interneurons determines hippocampal function and associated behavior. Neuron 53, 591-604.

Fuentealba, P., and Steriade, M. (2005). The reticular nucleus revisited: intrinsic and network properties of a thalamic pacemaker. Prog Neurobiol 75, 125-141.

Fukuda, T., and Kosaka, T. (2000). The dual network of GABAergic interneurons linked by both chemical and electrical synapses: a possible infrastructure of the cerebral cortex. Neurosci Res 38, 123-130.

Gandia, J.A., De Las Heras, S., Garcia, M., and Gimenez-Amaya, J.M. (1993). Afferent projections to the reticular thalamic nucleus from the globus pallidus and the substantia nigra in the rat. Brain Res Bull 32, 351-358.

Ganguly, K., Schinder, A.F., Wong, S.T., and Poo, M. (2001). GABA itself promotes the developmental switch of neuronal GABAergic responses from excitation to inhibition. Cell 105, 521-532.

Gao, B.X., Stricker, C., and Ziskind-Conhaim, L. (2001). Transition from GABAergic to glycinergic synaptic transmission in newly formed spinal networks. J Neurophysiol $86,492-502$.

Garin, N., Hornung, J.P., and Escher, G. (2002). Distribution of postsynaptic GABA(A) receptor aggregates in the deep cerebellar nuclei of normal and mutant mice. J Comp Neurol 447, 210-217.

Garner, C.C., Zhai, R.G., Gundelfinger, E.D., and Ziv, N.E. (2002). Molecular mechanisms of CNS synaptogenesis. Trends Neurosci 25, 243-251.

Gasnier, B. (2004). The SLC32 transporter, a key protein for the synaptic release of inhibitory amino acids. Pflugers Arch 447, 756-759.

Ge, S., Goh, E.L., Sailor, K.A., Kitabatake, Y., Ming, G.L., and Song, H. (2006). GABA regulates synaptic integration of newly generated neurons in the adult brain. Nature 439, 589-593. 
Ghazanfar, A.A., Chandrasekaran, C., and Logothetis, N.K. (2008). Interactions between the superior temporal sulcus and auditory cortex mediate dynamic face/voice integration in rhesus monkeys. J Neurosci 28, 4457-4469.

Gibbs, J.W., 3rd, Shumate, M.D., and Coulter, D.A. (1997). Differential epilepsyassociated alterations in postsynaptic $\operatorname{GABA}(\mathrm{A})$ receptor function in dentate granule and CA1 neurons. J Neurophysiol 77, 1924-1938.

Gillies, M.J., Traub, R.D., LeBeau, F.E., Davies, C.H., Gloveli, T., Buhl, E.H., and Whittington, M.A. (2002). A model of atropine-resistant theta oscillations in rat hippocampal area CA1. J Physiol 543, 779-793.

Glickfeld, L.L., and Scanziani, M. (2006). Distinct timing in the activity of cannabinoidsensitive and cannabinoid-insensitive basket cells. Nat Neurosci 9, 807-815.

Gloveli, T., Dugladze, T., Saha, S., Monyer, H., Heinemann, U., Traub, R.D., Whittington, M.A., and Buhl, E.H. (2005). Differential involvement of oriens/pyramidale interneurones in hippocampal network oscillations in vitro. $\mathrm{J}$ Physiol 562, 131-147.

Gomeza, J., Ohno, K., Hulsmann, S., Armsen, W., Eulenburg, V., Richter, D.W., Laube, B., and Betz, H. (2003). Deletion of the mouse glycine transporter 2 results in a hyperekplexia phenotype and postnatal lethality. Neuron 40,797-806.

Gras, C., Herzog, E., Bellenchi, G.C., Bernard, V., Ravassard, P., Pohl, M., Gasnier, B., Giros, B., and El Mestikawy, S. (2002). A third vesicular glutamate transporter expressed by cholinergic and serotoninergic neurons. J Neurosci 22, 5442-5451.

Gray, C.M., and Singer, W. (1989). Stimulus-specific neuronal oscillations in orientation columns of cat visual cortex. Proc Natl Acad Sci U S A 86, 1698-1702.

Gulyas, A.I., and Freund, T.F. (1996). Pyramidal cell dendrites are the primary targets of calbindin D28k-immunoreactive interneurons in the hippocampus. Hippocampus 6, 525-534.

Gulyas, A.I., Hajos, N., and Freund, T.F. (1996). Interneurons containing calretinin are specialized to control other interneurons in the rat hippocampus. J Neurosci 16, 3397-3411.

Gulyas, A.I., Megias, M., Emri, Z., and Freund, T.F. (1999). Total number and ratio of excitatory and inhibitory synapses converging onto single interneurons of different types in the CA1 area of the rat hippocampus. J Neurosci 19,10082-10097.

Gulyas, A.I., Sik, A., Payne, J.A., Kaila, K., and Freund, T.F. (2001). The KCl cotransporter, $\mathrm{KCC} 2$, is highly expressed in the vicinity of excitatory synapses in the rat hippocampus. Eur J Neurosci 13, 2205-2217.

Gulyas, A.I., Szabo, G.G., Ulbert, I., Holderith, N., Monyer, H., Erdelyi, F., Szabo, G., Freund, T.F., and Hajos, N. (2010). Parvalbumin-containing fast-spiking basket cells generate the field potential oscillations induced by cholinergic receptor activation in the hippocampus. J Neurosci 30, 15134-15145. 
Hajos, N., Katona, I., Naiem, S.S., MacKie, K., Ledent, C., Mody, I., and Freund, T.F. (2000). Cannabinoids inhibit hippocampal GABAergic transmission and network oscillations. Eur J Neurosci 12, 3239-3249.

Hajos, N., Palhalmi, J., Mann, E.O., Nemeth, B., Paulsen, O., and Freund, T.F. (2004). Spike timing of distinct types of GABAergic interneuron during hippocampal gamma oscillations in vitro. J Neurosci 24, 9127-9137.

Hajos, N., and Paulsen, O. (2009). Network mechanisms of gamma oscillations in the CA3 region of the hippocampus. Neural Netw 22, 1113-1119.

Hallanger, A.E., Levey, A.I., Lee, H.J., Rye, D.B., and Wainer, B.H. (1987). The origins of cholinergic and other subcortical afferents to the thalamus in the rat. $J$ Comp Neurol 262, 105-124.

Harkany, T., Holmgren, C., Hartig, W., Qureshi, T., Chaudhry, F.A., Storm-Mathisen, J., Dobszay, M.B., Berghuis, P., Schulte, G., Sousa, K.M., et al. (2004). Endocannabinoid-independent retrograde signaling at inhibitory synapses in layer $2 / 3$ of neocortex: involvement of vesicular glutamate transporter 3. J Neurosci 24, 49784988.

Harris, R.M. (1987). Axon collaterals in the thalamic reticular nucleus from thalamocortical neurons of the rat ventrobasal thalamus. J Comp Neurol 258, 397406.

Hashimoto, T., Arion, D., Unger, T., Maldonado-Aviles, J.G., Morris, H.M., Volk, D.W., Mirnics, K., and Lewis, D.A. (2008a). Alterations in GABA-related transcriptome in the dorsolateral prefrontal cortex of subjects with schizophrenia. Mol Psychiatry 13, 147-161.

Hashimoto, T., Bazmi, H.H., Mirnics, K., Wu, Q., Sampson, A.R., and Lewis, D.A. (2008b). Conserved regional patterns of GABA-related transcript expression in the neocortex of subjects with schizophrenia. Am J Psychiatry 165, 479-489.

Hashimoto, T., Volk, D.W., Eggan, S.M., Mirnics, K., Pierri, J.N., Sun, Z., Sampson, A.R., and Lewis, D.A. (2003). Gene expression deficits in a subclass of GABA neurons in the prefrontal cortex of subjects with schizophrenia. J Neurosci 23, 63156326.

Heck, N., Kilb, W., Reiprich, P., Kubota, H., Furukawa, T., Fukuda, A., and Luhmann, H.J. (2007). GABA-A receptors regulate neocortical neuronal migration in vitro and in vivo. Cereb Cortex 17, 138-148.

Hefft, S., and Jonas, P. (2005). Asynchronous GABA release generates long-lasting inhibition at a hippocampal interneuron-principal neuron synapse. Nat Neurosci 8, 1319-1328.

Herzog, E., Gilchrist, J., Gras, C., Muzerelle, A., Ravassard, P., Giros, B., Gaspar, P., and El Mestikawy, S. (2004). Localization of VGLUT3, the vesicular glutamate transporter type 3, in the rat brain. Neuroscience 123, 983-1002.

Hines, R.M., Wu, L., Hines, D.J., Steenland, H., Mansour, S., Dahlhaus, R., Singaraja, R.R., Cao, X., Sammler, E., Hormuzdi, S.G., et al. (2008). Synaptic 
imbalance, stereotypies, and impaired social interactions in mice with altered neuroligin 2 expression. J Neurosci 28, 6055-6067.

Hirzel, K., Muller, U., Latal, A.T., Hulsmann, S., Grudzinska, J., Seeliger, M.W., Betz, H., and Laube, B. (2006). Hyperekplexia phenotype of glycine receptor alpha1 subunit mutant mice identifies $\mathrm{Zn}(2+)$ as an essential endogenous modulator of glycinergic neurotransmission. Neuron 52, 679-690.

Hogema, B.M., Gupta, M., Senephansiri, H., Burlingame, T.G., Taylor, M., Jakobs, C., Schutgens, R.B., Froestl, W., Snead, O.C., Diaz-Arrastia, R., et al. (2001). Pharmacologic rescue of lethal seizures in mice deficient in succinate semialdehyde dehydrogenase. Nat Genet 29, 212-216.

Homanics, G.E., DeLorey, T.M., Firestone, L.L., Quinlan, J.J., Handforth, A., Harrison, N.L., Krasowski, M.D., Rick, C.E., Korpi, E.R., Makela, R., et al. (1997). Mice devoid of gamma-aminobutyrate type A receptor beta3 subunit have epilepsy, cleft palate, and hypersensitive behavior. Proc Natl Acad Sci U S A 94, 4143-4148.

Hormuzdi, S.G., Pais, I., LeBeau, F.E., Towers, S.K., Rozov, A., Buhl, E.H., Whittington, M.A., and Monyer, H. (2001). Impaired electrical signaling disrupts gamma frequency oscillations in connexin 36-deficient mice. Neuron 31, 487-495.

Houser, C.R., Vaughn, J.E., Barber, R.P., and Roberts, E. (1980). GABA neurons are the major cell type of the nucleus reticularis thalami. Brain Res 200, 341-354.

Hubner, C.A., Stein, V., Hermans-Borgmeyer, I., Meyer, T., Ballanyi, K., and Jentsch, T.J. (2001). Disruption of KCC2 reveals an essential role of K-Cl cotransport already in early synaptic inhibition. Neuron 30, 515-524.

Ivanov, S.V., Ward, J.M., Tessarollo, L., McAreavey, D., Sachdev, V., Fananapazir, L., Banks, M.K., Morris, N., Djurickovic, D., Devor-Henneman, D.E., et al. (2004). Cerebellar ataxia, seizures, premature death, and cardiac abnormalities in mice with targeted disruption of the Cacna2d2 gene. Am J Pathol 165, 1007-1018.

Jensen, O., Kaiser, J., and Lachaux, J.P. (2007). Human gamma-frequency oscillations associated with attention and memory. Trends Neurosci 30, 317-324.

Ji, F., Kanbara, N., and Obata, K. (1999). GABA and histogenesis in fetal and neonatal mouse brain lacking both the isoforms of glutamic acid decarboxylase. Neurosci Res 33, 187-194.

Jinno, S., Klausberger, T., Marton, L.F., Dalezios, Y., Roberts, J.D., Fuentealba, P., Bushong, E.A., Henze, D., Buzsaki, G., and Somogyi, P. (2007). Neuronal diversity in GABAergic long-range projections from the hippocampus. J Neurosci 27, 8790-8804.

Jokisch, D., and Jensen, O. (2007). Modulation of gamma and alpha activity during a working memory task engaging the dorsal or ventral stream. J Neurosci 27, 32443251.

Jonas, P., Bischofberger, J., Fricker, D., and Miles, R. (2004). Interneuron Diversity series: Fast in, fast out--temporal and spatial signal processing in hippocampal interneurons. Trends Neurosci 27, 30-40. 
Jonas, P., Bischofberger, J., and Sandkuhler, J. (1998). Corelease of two fast neurotransmitters at a central synapse. Science $281,419-424$.

Jouvet, M. (1969). Biogenic amines and the states of sleep. Science 163, 32-41.

Jun, K., Piedras-Renteria, E.S., Smith, S.M., Wheeler, D.B., Lee, S.B., Lee, T.G., Chin, H., Adams, M.E., Scheller, R.H., Tsien, R.W., and Shin, H.S. (1999). Ablation of $\mathrm{P} / \mathrm{Q}$-type $\mathrm{Ca}(2+)$ channel currents, altered synaptic transmission, and progressive ataxia in mice lacking the alpha(1A)-subunit. Proc Natl Acad Sci U S A 96, 1524515250.

Kalanithi, P.S.A., Zheng, W., Kataoka, Y., DiFiglia, M., Grantz, H., Saper, C.B., Schwartz, M.L., Leckman, J.F., and Vaccarino, F.M. (2005). Altered parvalbuminpositive neuron distribution in basal ganglia of individuals with Tourette syndrome. Proc Natl Acad Sci U S A 102, 13307-13312.

Karson, M.A., Tang, A.H., Milner, T.A., and Alger, B.E. (2009). Synaptic cross talk between perisomatic-targeting interneuron classes expressing cholecystokinin and parvalbumin in hippocampus. J Neurosci 29, 4140-4154.

Kash, S.F., Johnson, R.S., Tecott, L.H., Noebels, J.L., Mayfield, R.D., Hanahan, D., and Baekkeskov, S. (1997). Epilepsy in mice deficient in the $65-\mathrm{kDa}$ isoform of glutamic acid decarboxylase. Proc Natl Acad Sci U S A 94, 14060-14065.

Katona, I., Sperlagh, B., Sik, A., Kafalvi, A., Vizi, E.S., Mackie, K., and Freund, T.F. (1999). Presynaptically located CB1 cannabinoid receptors regulate GABA release from axon terminals of specific hippocampal interneurons. J Neurosci 19, 4544-4558.

Katona, I., Urban, G.M., Wallace, M., Ledent, C., Jung, K.M., Piomelli, D., Mackie, K., and Freund, T.F. (2006). Molecular composition of the endocannabinoid system at glutamatergic synapses. J Neurosci 26, 5628-5637.

Kawaguchi, Y., and Kubota, Y. (1997). GABAergic cell subtypes and their synaptic connections in rat frontal cortex. Cereb Cortex 7, 476-486.

Khalilov, I., Holmes, G.L., and Ben-Ari, Y. (2003). In vitro formation of a secondary epileptogenic mirror focus by interhippocampal propagation of seizures. Nat Neurosci 6, 1079-1085.

Khalilov, I., Khazipov, R., Esclapez, M., and Ben-Ari, Y. (1997). Bicuculline induces ictal seizures in the intact hippocampus recorded in vitro. Eur J Pharmacol 319, R5-6.

Khalilov, I., Le Van Quyen, M., Gozlan, H., and Ben-Ari, Y. (2005). Epileptogenic actions of GABA and fast oscillations in the developing hippocampus. Neuron 48, 787-796.

Khazipov, R., Esclapez, M., Caillard, O., Bernard, C., Khalilov, I., Tyzio, R., Hirsch, J., Dzhala, V., Berger, B., and Ben-Ari, Y. (2001). Early development of neuronal activity in the primate hippocampus in utero. J Neurosci $21,9770-9781$.

Kinney, J.W., Davis, C.N., Tabarean, I., Conti, B., Bartfai, T., and Behrens, M.M. (2006). A specific role for NR2A-containing NMDA receptors in the maintenance of 
parvalbumin and GAD67 immunoreactivity in cultured interneurons. J Neurosci 26, 1604-1615.

Klausberger, T. (2009). GABAergic interneurons targeting dendrites of pyramidal cells in the CA1 area of the hippocampus. Eur J Neurosci 30, 947-957.

Klausberger, T., Magill, P.J., Marton, L.F., Roberts, J.D., Cobden, P.M., Buzsaki, G., and Somogyi, P. (2003). Brain-state- and cell-type-specific firing of hippocampal interneurons in vivo. Nature $421,844-848$.

Klausberger, T., Marton, L.F., O'Neill, J., Huck, J.H., Dalezios, Y., Fuentealba, P., Suen, W.Y., Papp, E., Kaneko, T., Watanabe, M., et al. (2005). Complementary roles of cholecystokinin- and parvalbumin-expressing GABAergic neurons in hippocampal network oscillations. J Neurosci 25, 9782-9793.

Klausberger, T., and Somogyi, P. (2008). Neuronal diversity and temporal dynamics: the unity of hippocampal circuit operations. Science 321, 53-57.

Koenig, T., Lehmann, D., Saito, N., Kuginuki, T., Kinoshita, T., and Koukkou, M. (2001). Decreased functional connectivity of EEG theta-frequency activity in firstepisode, neuroleptic-naive patients with schizophrenia: preliminary results. Schizophr Res 50, 55-60.

Kolmac, C.I., and Mitrofanis, J. (1998). Patterns of brainstem projection to the thalamic reticular nucleus. J Comp Neurol 396, 531-543.

Korotkova, T., Fuchs, E.C., Ponomarenko, A., von Engelhardt, J., and Monyer, H. (2010). NMDA receptor ablation on parvalbumin-positive interneurons impairs hippocampal synchrony, spatial representations, and working memory. Neuron 68, 557-569.

Krnjevic, K., and Schwartz, S. (1967). The action of gamma-aminobutyric acid on cortical neurones. Exp Brain Res 3, 320-336.

Lau, D., Vega-Saenz de Miera, E.C., Contreras, D., Ozaita, A., Harvey, M., Chow, A., Noebels, J.L., Paylor, R., Morgan, J.I., Leonard, C.S., and Rudy, B. (2000). Impaired fast-spiking, suppressed cortical inhibition, and increased susceptibility to seizures in mice lacking Kv3.2 K+ channel proteins. J Neurosci 20, 9071-9085.

LeBeau, F.E., Towers, S.K., Traub, R.D., Whittington, M.A., and Buhl, E.H. (2002). Fast network oscillations induced by potassium transients in the rat hippocampus in vitro. J Physiol 542, 167-179.

Lee, C.R., and Tepper, J.M. (2007). Morphological and physiological properties of parvalbumin- and calretinin-containing gamma-aminobutyric acidergic neurons in the substantia nigra. J Comp Neurol 500, 958-972.

Leinekugel, X., Medina, I., Khalilov, I., Ben-Ari, Y., and Khazipov, R. (1997). Ca2+ oscillations mediated by the synergistic excitatory actions of $\operatorname{GABA}(A)$ and NMDA receptors in the neonatal hippocampus. Neuron 18, 243-255. 
Leinekugel, X., Tseeb, V., Ben-Ari, Y., and Bregestovski, P. (1995). Synaptic GABAA activation induces $\mathrm{Ca} 2+$ rise in pyramidal cells and interneurons from rat neonatal hippocampal slices. J Physiol 487 ( Pt 2), 319-329.

Leone, D.P., Genoud, S., Atanasoski, S., Grausenburger, R., Berger, P., Metzger, D., Macklin, W.B., Chambon, P., and Suter, U. (2003). Tamoxifen-inducible glia-specific Cre mice for somatic mutagenesis in oligodendrocytes and Schwann cells. Mol Cell Neurosci 22, 430-440.

Lewis, D.A., Hashimoto, T., and Volk, D.W. (2005). Cortical inhibitory neurons and schizophrenia. Nat Rev Neurosci 6, 312-324.

Lim, R., Alvarez, F.J., and Walmsley, B. (2000). GABA mediates presynaptic inhibition at glycinergic synapses in a rat auditory brainstem nucleus. J Physiol 525 Pt 2, 447-459.

Linnemann, C., Sultan, F., Pedroarena, C.M., Schwarz, C., and Thier, P. (2004). Lurcher mice exhibit potentiation of $\operatorname{GABA}(\mathrm{A})$-receptor-mediated conductance in cerebellar nuclei neurons in close temporal relationship to Purkinje cell death. J Neurophysiol 91, 1102-1107.

Liu, M., Pleasure, S.J., Collins, A.E., Noebels, J.L., Naya, F.J., Tsai, M.J., and Lowenstein, D.H. (2000). Loss of BETA2/NeuroD leads to malformation of the dentate gyrus and epilepsy. Proc Natl Acad Sci U S A 97, 865-870.

Liu, P., Jenkins, N.A., and Copeland, N.G. (2003). A highly efficient recombineeringbased method for generating conditional knockout mutations. Genome Res 13, 476484.

Liu, X.B., and Jones, E.G. (1999). Predominance of corticothalamic synaptic inputs to thalamic reticular nucleus neurons in the rat. J Comp Neurol 414, 67-79.

Lobe, C.G., Koop, K.E., Kreppner, W., Lomeli, H., Gertsenstein, M., and Nagy, A. (1999). Z/AP, a double reporter for cre-mediated recombination. Dev Biol 208, 281292.

Lopez-Bendito, G., Lujan, R., Shigemoto, R., Ganter, P., Paulsen, O., and Molnar, Z. (2003). Blockade of $\mathrm{GABA}(\mathrm{B})$ receptors alters the tangential migration of cortical neurons. Cereb Cortex 13, 932-942.

LoTurco, J.J., Owens, D.F., Heath, M.J., Davis, M.B., and Kriegstein, A.R. (1995). GABA and glutamate depolarize cortical progenitor cells and inhibit DNA synthesis. Neuron 15, 1287-1298.

Loup, F., Wieser, H.G., Yonekawa, Y., Aguzzi, A., and Fritschy, J.M. (2000). Selective alterations in GABAA receptor subtypes in human temporal lobe epilepsy. J Neurosci 20, 5401-5419.

Lu, T., Rubio, M.E., and Trussell, L.O. (2008). Glycinergic transmission shaped by the corelease of GABA in a mammalian auditory synapse. Neuron $57,524-535$. 
Lubke, J. (1993). Morphology of neurons in the thalamic reticular nucleus (TRN) of mammals as revealed by intracellular injections into fixed brain slices. J Comp Neurol $329,458-471$.

Ludwig, A., Li, H., Saarma, M., Kaila, K., and Rivera, C. (2003). Developmental upregulation of KCC2 in the absence of GABAergic and glutamatergic transmission. Eur J Neurosci 18, 3199-3206.

Maccaferri, G., and Lacaille, J.C. (2003). Interneuron Diversity series: Hippocampal interneuron classifications--making things as simple as possible, not simpler. Trends Neurosci 26, 564-571.

Mainy, N., Kahane, P., Minotti, L., Hoffmann, D., Bertrand, O., and Lachaux, J.P. (2007). Neural correlates of consolidation in working memory. Hum Brain Mapp 28, 183-193.

Mann, E.O., and Mody, I. (2010). Control of hippocampal gamma oscillation frequency by tonic inhibition and excitation of interneurons. Nat Neurosci 13, 205212.

Mann, E.O., and Paulsen, O. (2005). Mechanisms underlying gamma ('40 Hz') network oscillations in the hippocampus--a mini-review. Prog Biophys Mol Biol 87, 67-76.

Mann, E.O., Radcliffe, C.A., and Paulsen, O. (2005a). Hippocampal gammafrequency oscillations: from interneurones to pyramidal cells, and back. J Physiol $562,55-63$.

Mann, E.O., Suckling, J.M., Hajos, N., Greenfield, S.A., and Paulsen, O. (2005b). Perisomatic feedback inhibition underlies cholinergically induced fast network oscillations in the rat hippocampus in vitro. Neuron 45, 105-117.

Marin-Padilla, M. (1969). Origin of the pericellular baskets of the pyramidal cells of the human motor cortex: a Golgi study. Brain Res 14, 633-646.

Markram, H., Toledo-Rodriguez, M., Wang, Y., Gupta, A., Silberberg, G., and Wu, C. (2004). Interneurons of the neocortical inhibitory system. Nat Rev Neurosci 5, 793807.

Marsicano, G., and Lutz, B. (1999). Expression of the cannabinoid receptor CB1 in distinct neuronal subpopulations in the adult mouse forebrain. Eur $\mathrm{J}$ Neurosci 11 , 4213-4225.

Mathern, G.W., Babb, T.L., Pretorius, J.K., and Leite, J.P. (1995). Reactive synaptogenesis and neuron densities for neuropeptide $\mathrm{Y}$, somatostatin, and glutamate decarboxylase immunoreactivity in the epileptogenic human fascia dentata. J Neurosci 15, 3990-4004.

Matyas, F., Freund, T.F., and Gulyas, A.I. (2004). Convergence of excitatory and inhibitory inputs onto CCK-containing basket cells in the CA1 area of the rat hippocampus. Eur J Neurosci 19, 1243-1256. 
McBain, C.J., and Fisahn, A. (2001). Interneurons unbound. Nat Rev Neurosci 2, 1123.

McCormick, D.A., Connors, B.W., Lighthall, J.W., and Prince, D.A. (1985). Comparative electrophysiology of pyramidal and sparsely spiny stellate neurons of the neocortex. J Neurophysiol 54, 782-806.

McCormick, D.A., and Contreras, D. (2001). On the cellular and network bases of epileptic seizures. Annu Rev Physiol 63, 815-846.

Mclntire, S.L., Jorgensen, E., and Horvitz, H.R. (1993). Genes required for GABA function in Caenorhabditis elegans. Nature 364, 334-337.

McIntire, S.L., Reimer, R.J., Schuske, K., Edwards, R.H., and Jorgensen, E.M. (1997). Identification and characterization of the vesicular GABA transporter. Nature $389,870-876$.

Mody, I., and Pearce, R.A. (2004). Diversity of inhibitory neurotransmission through GABA(A) receptors. Trends Neurosci 27, 569-575.

Moechars, D., Weston, M.C., Leo, S., Callaerts-Vegh, Z., Goris, I., Daneels, G., Buist, A., Cik, M., van der Spek, P., Kass, S., et al. (2006). Vesicular glutamate transporter VGLUT2 expression levels control quantal size and neuropathic pain. J Neurosci 26, 12055-12066.

Montgomery, S.M., and Buzsaki, G. (2007). Gamma oscillations dynamically couple hippocampal CA3 and CA1 regions during memory task performance. Proc Natl Acad Sci U S A 104, 14495-14500.

Monyer, H., and Markram, H. (2004). Interneuron Diversity series: Molecular and genetic tools to study GABAergic interneuron diversity and function. Trends Neurosci $27,90-97$.

Morales, M., and Bloom, F.E. (1997). The 5-HT3 receptor is present in different subpopulations of GABAergic neurons in the rat telencephalon. J Neurosci 17, 31573167.

Morimoto, K., Fahnestock, M., and Racine, R.J. (2004). Kindling and status epilepticus models of epilepsy: rewiring the brain. Prog Neurobiol 73, 1-60.

Muzumdar, M.D., Tasic, B., Miyamichi, K., Li, L., and Luo, L. (2007). A global doublefluorescent Cre reporter mouse. Genesis 45, 593-605.

Nagy, A. (2000). Cre recombinase: the universal reagent for genome tailoring. Genesis 26, 99-109.

Nakamura, T., Colbert, M.C., and Robbins, J. (2006). Neural crest cells retain multipotential characteristics in the developing valves and label the cardiac conduction system. Circ Res 98, 1547-1554.

Neu, A., Foldy, C., and Soltesz, I. (2007). Postsynaptic origin of CB1-dependent tonic inhibition of GABA release at cholecystokinin-positive basket cell to pyramidal cell synapses in the CA1 region of the rat hippocampus. J Physiol 578, 233-247. 
Noebels, J.L. (2003). The biology of epilepsy genes. Annu Rev Neurosci 26, 599625.

Novak, A., Guo, C., Yang, W., Nagy, A., and Lobe, C.G. (2000). Z/EG, a double reporter mouse line that expresses enhanced green fluorescent protein upon Cremediated excision. Genesis 28, 147-155.

O'Brien, J.A., and Berger, A.J. (1999). Cotransmission of GABA and glycine to brain stem motoneurons. J Neurophysiol 82, 1638-1641.

Ohara, P.T., and Havton, L.A. (1996). Dendritic arbors of neurons from different regions of the rat thalamic reticular nucleus share a similar orientation. Brain Res 731, 236-240.

Okabe, S. (2007). Molecular anatomy of the postsynaptic density. Mol Cell Neurosci $34,503-518$.

Owens, D.F., Boyce, L.H., Davis, M.B., and Kriegstein, A.R. (1996). Excitatory GABA responses in embryonic and neonatal cortical slices demonstrated by gramicidin perforated-patch recordings and calcium imaging. J Neurosci 16, 6414-6423.

Owens, D.F., and Kriegstein, A.R. (2002a). Developmental neurotransmitters? Neuron 36, 989-991.

Owens, D.F., and Kriegstein, A.R. (2002b). Is there more to GABA than synaptic inhibition? Nat Rev Neurosci 3, 715-727.

Pare, D., Hazrati, L.N., Parent, A., and Steriade, M. (1990). Substantia nigra pars reticulata projects to the reticular thalamic nucleus of the cat: a morphological and electrophysiological study. Brain Res 535, 139-146.

Pawelzik, H., Hughes, D.I., and Thomson, A.M. (2002). Physiological and morphological diversity of immunocytochemically defined parvalbumin- and cholecystokinin-positive interneurones in CA1 of the adult rat hippocampus. J Comp Neurol 443, 346-367.

Penttonen, M., Kamondi, A., Acsady, L., and Buzsaki, G. (1998). Gamma frequency oscillation in the hippocampus of the rat: intracellular analysis in vivo. Eur $\mathrm{J}$ Neurosci 10, 718-728.

Phillips, R. (1960). 'Lurcher', a new gene in linkage group XI of the house mouse. Journ Genet 57, 35-42-42.

Pinault, D. (2004). The thalamic reticular nucleus: structure, function and concept. Brain Res Brain Res Rev 46, 1-31.

Pinault, D. (2011). Dysfunctional thalamus-related networks in schizophrenia. Schizophr Bull 37, 238-243.

Pinault, D., and Deschenes, M. (1998). Projection and innervation patterns of individual thalamic reticular axons in the thalamus of the adult rat: a threedimensional, graphic, and morphometric analysis. J Comp Neurol 391, 180-203. 
Pinault, D., Smith, Y., and Deschenes, M. (1997). Dendrodendritic and axoaxonic synapses in the thalamic reticular nucleus of the adult rat. J Neurosci 17, 3215-3233.

Prior, P., Schmitt, B., Grenningloh, G., Pribilla, I., Multhaup, G., Beyreuther, K., Maulet, Y., Werner, P., Langosch, D., Kirsch, J., and et al. (1992). Primary structure and alternative splice variants of gephyrin, a putative glycine receptor-tubulin linker protein. Neuron 8, 1161-1170.

Prosser, H.M., Gill, C.H., Hirst, W.D., Grau, E., Robbins, M., Calver, A., Soffin, E.M., Farmer, C.E., Lanneau, C., Gray, J., et al. (2001). Epileptogenesis and enhanced prepulse inhibition in GABA(B1)-deficient mice. Mol Cell Neurosci 17, 1059-1070.

Pyott, S.J., and Rosenmund, C. (2002). The effects of temperature on vesicular supply and release in autaptic cultures of rat and mouse hippocampal neurons. J Physiol 539, 523-535.

Racz, A., Ponomarenko, A.A., Fuchs, E.C., and Monyer, H. (2009). Augmented hippocampal ripple oscillations in mice with reduced fast excitation onto parvalbuminpositive cells. J Neurosci 29, 2563-2568.

Represa, A., and Ben-Ari, Y. (2005). Trophic actions of GABA on neuronal development. Trends Neurosci 28, 278-283.

Represa, A., Jorquera, I., Le Gal La Salle, G., and Ben-Ari, Y. (1993a). Epilepsy induced collateral sprouting of hippocampal mossy fibers: does it induce the development of ectopic synapses with granule cell dendrites? Hippocampus 3, 257268.

Represa, A., Pollard, H., Moreau, J., Ghilini, G., Khrestchatisky, M., and Ben-Ari, Y. (1993b). Mossy fiber sprouting in epileptic rats is associated with a transient increased expression of alpha-tubulin. Neurosci Lett 156, 149-152.

Represa, A., Tremblay, E., and Benari, Y. (1987). Kainate Binding-Sites in the Hippocampal Mossy Fibers - Localization and Plasticity. Neuroscience 20, 739-748.

Ribar, T.J., Rodriguiz, R.M., Khiroug, L., Wetsel, W.C., Augustine, G.J., and Means, A.R. (2000). Cerebellar defects in Ca2+/calmodulin kinase IV-deficient mice. J Neurosci 20, RC107.

Rivera, C., Voipio, J., Payne, J.A., Ruusuvuori, E., Lahtinen, H., Lamsa, K., Pirvola, U., Saarma, M., and Kaila, K. (1999). The K+/Cl- co-transporter KCC2 renders GABA hyperpolarizing during neuronal maturation. Nature 397, 251-255.

Rosahl, T.W., Spillane, D., Missler, M., Herz, J., Selig, D.K., Wolff, J.R., Hammer, R.E., Malenka, R.C., and Sudhof, T.C. (1995). Essential functions of synapsins I and II in synaptic vesicle regulation. Nature 375, 488-493.

Sagne, C., El Mestikawy, S., Isambert, M.F., Hamon, M., Henry, J.P., Giros, B., and Gasnier, B. (1997). Cloning of a functional vesicular GABA and glycine transporter by screening of genome databases. FEBS Lett 417, 177-183. 
Schuler, V., Luscher, C., Blanchet, C., Klix, N., Sansig, G., Klebs, K., Schmutz, M., Heid, J., Gentry, C., Urban, L., et al. (2001). Epilepsy, hyperalgesia, impaired memory, and loss of pre- and postsynaptic $G A B A(B)$ responses in mice lacking $\operatorname{GABA}(B(1))$. Neuron 31, 47-58.

Schwarzer, C., Tsunashima, K., Wanzenbock, C., Fuchs, K., Sieghart, W., and Sperk, G. (1997). GABA(A) receptor subunits in the rat hippocampus II: altered distribution in kainic acid-induced temporal lobe epilepsy. Neuroscience 80, 10011017.

Sernagor, E., Chabrol, F., Bony, G., and Cancedda, L. (2010). GABAergic control of neurite outgrowth and remodeling during development and adult neurogenesis: general rules and differences in diverse systems. Front Cell Neurosci 4, 11.

Sik, A., Penttonen, M., Ylinen, A., and Buzsaki, G. (1995). Hippocampal CA1 interneurons: an in vivo intracellular labeling study. J Neurosci 15, 6651-6665.

Smart, T.G. (2010). Handling accumulated internal Cl- at inhibitory synapses. Nat Neurosci 13, 1043-1044.

Sohal, V.S., Zhang, F., Yizhar, O., and Deisseroth, K. (2009). Parvalbumin neurons and gamma rhythms enhance cortical circuit performance. Nature 459, 698-702.

Solbach, S., and Celio, M.R. (1991). Ontogeny of the calcium binding protein parvalbumin in the rat nervous system. Anat Embryol (Berl) 184, 103-124.

Somogyi, J., Baude, A., Omori, Y., Shimizu, H., El Mestikawy, S., Fukaya, M., Shigemoto, R., Watanabe, M., and Somogyi, P. (2004). GABAergic basket cells expressing cholecystokinin contain vesicular glutamate transporter type 3 (VGLUT3) in their synaptic terminals in hippocampus and isocortex of the rat. Eur $\mathrm{J}$ Neurosci 19, 552-569.

Somogyi, P. (1977). A specific 'axo-axonal' interneuron in the visual cortex of the rat. Brain Res 136, 345-350.

Somogyi, P., and Klausberger, T. (2005). Defined types of cortical interneurone structure space and spike timing in the hippocampus. J Physiol 562, 9-26.

Soriano, P. (1999). Generalized lacZ expression with the ROSA26 Cre reporter strain. Nat Genet 21, 70-71.

Spencer, K.M., Niznikiewicz, M.A., Nestor, P.G., Shenton, M.E., and McCarley, R.W. (2009). Left auditory cortex gamma synchronization and auditory hallucination symptoms in schizophrenia. BMC Neurosci 10, 85.

Spreafico, R., Battaglia, G., and Frassoni, C. (1991). The reticular thalamic nucleus (RTN) of the rat: cytoarchitectural, Golgi, immunocytochemical, and horseradish peroxidase study. J Comp Neurol 304, 478-490.

Staley, K., and Smith, R. (2001). A new form of feedback at the GABA(A) receptor. Nat Neurosci 4, 674-676. 
Stein, V., Hermans-Borgmeyer, I., Jentsch, T.J., and Hubner, C.A. (2004). Expression of the $\mathrm{KCl}$ cotransporter $\mathrm{KCC} 2$ parallels neuronal maturation and the emergence of low intracellular chloride. J Comp Neurol 468, 57-64.

Steriade, M. (1999). Coherent oscillations and short-term plasticity in corticothalamic networks. Trends Neurosci 22, 337-345.

Steriade, M. (2005). Sleep, epilepsy and thalamic reticular inhibitory neurons. Trends Neurosci 28, 317-324.

Steriade, M., and Contreras, D. (1995). Relations between cortical and thalamic cellular events during transition from sleep patterns to paroxysmal activity. J Neurosci $15,623-642$.

Steriade, M., and Contreras, D. (1998). Spike-wave complexes and fast components of cortically generated seizures. I. Role of neocortex and thalamus. J Neurophysiol $80,1439-1455$.

Steriade, M., Domich, L., Oakson, G., and Deschenes, M. (1987). The deafferented reticular thalamic nucleus generates spindle rhythmicity. J Neurophysiol 57, 260-273.

Steriade, M., McCormick, D.A., and Sejnowski, T.J. (1993). Thalamocortical oscillations in the sleeping and aroused brain. Science 262, 679-685.

Steriade, M., and Timofeev, I. (2003). Neuronal plasticity in thalamocortical networks during sleep and waking oscillations. Neuron 37, 563-576.

Sudhof, T.C. (2004). The synaptic vesicle cycle. Annu Rev Neurosci 27, 509-547.

Sultan, F., Konig, T., Mock, M., and Thier, P. (2002). Quantitative organization of neurotransmitters in the deep cerebellar nuclei of the Lurcher mutant. J Comp Neurol 452, 311-323.

Svoboda, K.R., Adams, C.E., and Lupica, C.R. (1999). Opioid receptor subtype expression defines morphologically distinct classes of hippocampal interneurons. J Neurosci 19, 85-95.

Tallon-Baudry, C., Bertrand, O., Henaff, M.A., Isnard, J., and Fischer, C. (2005). Attention modulates gamma-band oscillations differently in the human lateral occipital cortex and fusiform gyrus. Cereb Cortex 15, 654-662.

Teune, T.M., van der Burg, J., de Zeeuw, C.I., Voogd, J., and Ruigrok, T.J. (1998). Single Purkinje cell can innervate multiple classes of projection neurons in the cerebellar nuclei of the rat: a light microscopic and ultrastructural triple-tracer study in the rat. J Comp Neurol 392, 164-178.

Teune, T.M., van der Burg, J., and Ruigrok, T.J. (1995). Cerebellar projections to the red nucleus and inferior olive originate from separate populations of neurons in the rat: a non-fluorescent double labeling study. Brain Res 673, 313-319.

Tiesinga, P., and Sejnowski, T.J. (2009). Cortical enlightenment: are attentional gamma oscillations driven by ING or PING? Neuron 63, 727-732. 
Tiitinen, H., Sinkkonen, J., Reinikainen, K., Alho, K., Lavikainen, J., and Naatanen, R. (1993). Selective attention enhances the auditory $40-\mathrm{Hz}$ transient response in humans. Nature 364, 59-60.

Titz, S., Hans, M., Kelsch, W., Lewen, A., Swandulla, D., and Misgeld, U. (2003). Hyperpolarizing inhibition develops without trophic support by GABA in cultured rat midbrain neurons. J Physiol 550, 719-730.

Traub, R.D., Bibbig, A., Fisahn, A., LeBeau, F.E., Whittington, M.A., and Buhl, E.H. (2000). A model of gamma-frequency network oscillations induced in the rat CA3 region by carbachol in vitro. Eur J Neurosci 12, 4093-4106.

Traub, R.D., Bibbig, A., LeBeau, F.E., Buhl, E.H., and Whittington, M.A. (2004). Cellular mechanisms of neuronal population oscillations in the hippocampus in vitro. Annu Rev Neurosci 27, 247-278.

Tukker, J.J., Fuentealba, P., Hartwich, K., Somogyi, P., and Klausberger, T. (2007). Cell type-specific tuning of hippocampal interneuron firing during gamma oscillations in vivo. J Neurosci 27, 8184-8189.

Tyzio, R., Represa, A., Jorquera, I., Ben-Ari, Y., Gozlan, H., and Aniksztejn, L. (1999). The establishment of GABAergic and glutamatergic synapses on CA1 pyramidal neurons is sequential and correlates with the development of the apical dendrite. J Neurosci 19, 10372-10382.

Uhlhaas, P.J., Linden, D.E., Singer, W., Haenschel, C., Lindner, M., Maurer, K., and Rodriguez, E. (2006). Dysfunctional long-range coordination of neural activity during Gestalt perception in schizophrenia. J Neurosci 26, 8168-8175.

Uhlhaas, P.J., and Singer, W. (2010). Abnormal neural oscillations and synchrony in schizophrenia. Nat Rev Neurosci 11, 100-113.

Uusisaari, M., Obata, K., and Knopfel, T. (2007). Morphological and electrophysiological properties of GABAergic and non-GABAergic cells in the deep cerebellar nuclei. J Neurophysiol 97, 901-911.

van Hooft, J.A., Giuffrida, R., Blatow, M., and Monyer, H. (2000). Differential expression of group I metabotropic glutamate receptors in functionally distinct hippocampal interneurons. J Neurosci 20, 3544-3551.

van Vliet, E.A., Aronica, E., Tolner, E.A., Lopes da Silva, F.H., and Gorter, J.A. (2004). Progression of temporal lobe epilepsy in the rat is associated with immunocytochemical changes in inhibitory interneurons in specific regions of the hippocampal formation. Exp Neurol 187, 367-379.

van Vugt, M.K., Schulze-Bonhage, A., Litt, B., Brandt, A., and Kahana, M.J. (2010). Hippocampal gamma oscillations increase with memory load. J Neurosci 30, 26942699.

Vanderwolf, C.H. (1969). Hippocampal electrical activity and voluntary movement in the rat. Electroencephalogr Clin Neurophysiol 26, 407-418. 
Varga, V., Losonczy, A., Zemelman, B.V., Borhegyi, Z., Nyiri, G., Domonkos, A., Hangya, B., Holderith, N., Magee, J.C., and Freund, T.F. (2009). Fast synaptic subcortical control of hippocampal circuits. Science 326, 449-453.

Vida, I., Bartos, M., and Jonas, P. (2006). Shunting inhibition improves robustness of gamma oscillations in hippocampal interneuron networks by homogenizing firing rates. Neuron 49, 107-117.

Volk, D., Austin, M., Pierri, J., Sampson, A., and Lewis, D. (2001). GABA transporter1 mRNA in the prefrontal cortex in schizophrenia: decreased expression in a subset of neurons. Am J Psychiatry 158, 256-265.

Voogd, J., and Glickstein, M. (1998). The anatomy of the cerebellum. Trends Neurosci 21, 370-375.

Wallen-Mackenzie, A., Gezelius, H., Thoby-Brisson, M., Nygard, A., Enjin, A., Fujiyama, F., Fortin, G., and Kullander, K. (2006). Vesicular glutamate transporter 2 is required for central respiratory rhythm generation but not for locomotor central pattern generation. J Neurosci 26, 12294-12307.

Wang, X.J. (2010). Neurophysiological and computational principles of cortical rhythms in cognition. Physiol Rev 90, 1195-1268.

Wendling, F., Bartolomei, F., Bellanger, J.J., and Chauvel, P. (2002). Epileptic fast activity can be explained by a model of impaired GABAergic dendritic inhibition. Eur $\mathrm{J}$ Neurosci 15, 1499-1508.

Werman, R., Davidoff, R.A., and Aprison, M.H. (1968). Inhibitory of glycine on spinal neurons in the cat. J Neurophysiol 31, 81-95.

Whittington, M.A., Traub, R.D., and Jefferys, J.G. (1995). Synchronized oscillations in interneuron networks driven by metabotropic glutamate receptor activation. Nature $373,612-615$.

Wilson, R.I., and Nicoll, R.A. (2001). Endogenous cannabinoids mediate retrograde signalling at hippocampal synapses. Nature 410, 588-592.

Wittner, L., Eross, L., Czirjak, S., Halasz, P., Freund, T.F., and Magloczky, Z. (2005). Surviving CA1 pyramidal cells receive intact perisomatic inhibitory input in the human epileptic hippocampus. Brain 128, 138-152.

Wittner, L., Magloczky, Z., Borhegyi, Z., Halasz, P., Toth, S., Eross, L., Szabo, Z., and Freund, T.F. (2001). Preservation of perisomatic inhibitory input of granule cells in the epileptic human dentate gyrus. Neuroscience 108, 587-600.

Wojcik, S.M., Katsurabayashi, S., Guillemin, I., Friauf, E., Rosenmund, C., Brose, N., and Rhee, J.S. (2006). A shared vesicular carrier allows synaptic corelease of GABA and glycine. Neuron 50, 575-587.

Wojcik, S.M., Rhee, J.S., Herzog, E., Sigler, A., Jahn, R., Takamori, S., Brose, N., and Rosenmund, C. (2004). An essential role for vesicular glutamate transporter 1 
(VGLUT1) in postnatal development and control of quantal size. Proc Natl Acad Sci U S A 101, 7158-7163.

Wulff, P., Ponomarenko, A.A., Bartos, M., Korotkova, T.M., Fuchs, E.C., Bahner, F., Both, M., Tort, A.B., Kopell, N.J., Wisden, W., and Monyer, H. (2009). Hippocampal theta rhythm and its coupling with gamma oscillations require fast inhibition onto parvalbumin-positive interneurons. Proc Natl Acad Sci U S A 106, 3561-3566.

Xu, X., Roby, K.D., and Callaway, E.M. (2010). Immunochemical characterization of inhibitory mouse cortical neurons: three chemically distinct classes of inhibitory cells. J Comp Neurol 518, 389-404.

Yamada, J., Okabe, A., Toyoda, H., Kilb, W., Luhmann, H.J., and Fukuda, A. (2004). $\mathrm{Cl}$ - uptake promoting depolarizing GABA actions in immature rat neocortical neurones is mediated by NKCC1. J Physiol 557, 829-841.

Yang, X.W., Model, P., and Heintz, N. (1997). Homologous recombination based modification in Escherichia coli and germline transmission in transgenic mice of a bacterial artificial chromosome. Nat Biotechnol 15, 859-865.

Zhang, L., and Jones, E.G. (2004). Corticothalamic inhibition in the thalamic reticular nucleus. J Neurophysiol 91, 759-766.

Zheng, N., and Raman, I.M. (2010). Synaptic inhibition, excitation, and plasticity in neurons of the cerebellar nuclei. Cerebellum 9, 56-66. 


\section{Curriculum Vitae}

CONTACT DETAILS

Max-Planck-Institute of Experimental Medicine

Herrmann-Rein-Straße 3

37075 Göttingen

Germany

Phone: 0551 / 3899693

\begin{tabular}{ll} 
EDUCATION & \\
\hline $\mathbf{0 9 / 1 9 9 8 - 0 6 / 2 0 0 1}$ & $\begin{array}{l}\text { Secondary School } \\
\text { "Berufliches Schulzentrum" for Economics Kamenz }\end{array}$ \\
$10 / 2002-09 / 2005$ & $\begin{array}{l}\text { BSc Program Molecular Biotechnology } \\
\text { Technische Universität Dresden }\end{array}$ \\
$10 / 2006-09 / 2007$ & $\begin{array}{l}\text { MSc/PhD Program Molecular Biology } \\
\text { Georg-August-Universität and International Max Planck } \\
\text { Research School, Göttingen }\end{array}$
\end{tabular}

WORKING EXPERIENCE (IN REVERSE ORDER)

\begin{tabular}{|c|c|}
\hline Since 10/2007 & $\begin{array}{l}\text { PhD Thesis } \\
\text { International Max Planck Research School } \\
\text { MPI of Experimental Medicine - Dept. Molecular Neurobiology } \\
\text { - Thesis Title: Genetic Targeting and Analysis of Parvalbumin and } \\
\text { VGLUT3 Expressing Inhibitory Neurons } \\
\text { - Thesis committee: } 1^{\text {st. }} \text { : Dr. S. Wojcik, } 2^{\text {nd }} \text { : Prof. Dr. E. Neher, } 3^{\text {rd }} \text { : } \\
\text { Prof. Dr. A. Mansouri }\end{array}$ \\
\hline $01 / 2007-06 / 2007$ & $\begin{array}{l}\text { Laboratory rotation projects } \quad \text { Göttingen, Germany } \\
\text { - MPI for Biophysical Chemistry - Dept. Molecular Developmental } \\
\text { Biology, Molecular Physiology } \\
\text { - } \quad \text { MPI for Experimental Medicine - Dept. of Molecular Neurobiology } \\
\text { - MPI for Biophysical Chemistry - X-Ray Crystallography }\end{array}$ \\
\hline $10 / 2005-09 / 2006$ & $\begin{array}{l}\text { Hanson Institute, Royal Adelaide Hospital Adelaide, Australia } \\
\text { - Voluntary work experience: Studies to the immune activation in } \\
\text { patients with functional gastrointestinal disorders (Dept. of } \\
\text { Gastroenterology and Hepatology, Nerve-Gut-Research } \\
\text { Laboratory) }\end{array}$ \\
\hline $04 / 2005-09 / 2005$ & $\begin{array}{l}\text { Bachelor Thesis Dresden, Germany } \\
\text { Max-Bergmann-Center of Biomaterials Dresden, Institute of } \\
\text { Materials Science, Biomaterials } \\
\text { - Title: Studies of the influence of artificial extracellular matrices on } \\
\text { the gene expression of primary rat calvaria cells in-vitro }\end{array}$ \\
\hline $08 / 2004-09 / 2004$ & $\begin{array}{l}\text { ABX GmbH } \\
\text { - Vadeberg, Germany } \\
\text { cleantary internship: Synthesis of Mannose Triflate under defined } \\
\text { cleonditions }\end{array}$ \\
\hline
\end{tabular}


09/2009 European Synapse Summer School

PENS Trainings Center, Bordeaux, France

SCHOLARSHIPS

09/2006- 08/2007

Stipend International Max Planck Research School

\section{PUBLICATIONS}

Liebregts $T^{*}$., Adam B*., Bredack C., Gururatsakul M., Pilkington K.R., Brierley .SM., Blackshaw L.A., Gerken G., Talley N.J., Holtmann G. (2011), Small bowel homing T cells are associated with symptoms and delayed gastric emptying in functional dyspepsia. Am J Gastroenterol. Jan 18

Bredack C., Monyer H., Wojcik S. M. (2010). Genetic analysis of the function of parvalbumin positive interneurons. FENS Abstr., vol. 5, 195.5

Liebregts T., Adam B., Bredack C., Röth A., Heinzel S., Lester S., Downie-Doyle S., Smith E., Drew P., Talley N.J., Holtmann G. (2007). Immune activation in patients with irritable bowel syndrome. Gastroenterology 132, 913-920

NON-SCIENTIFIC ACTIVITIES

2009 and $2010 \quad$ Organisation Committee

- " $66^{\text {th }}$ and $7^{\text {th }}$ Horizons in Molecular Biology", Göttingen, Germany 